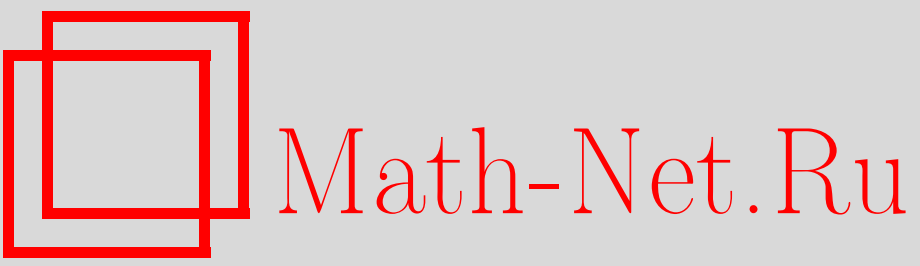

М. О. Корпусов, Г. И. Шляпугин, О разрушении решений задач Коши для одного класса нелинейных уравнений теории ферритов, Итоги науки и техн. Сер. Соврем. мат. и ее прил. Темат. обз., 2020, том 185, 79-131

DOI: https://doi.org/10.36535/0233-6723-2020-185-79-131

Использование Общероссийского математического портала Math-Net.Ru подразумевает, что вы прочитали и согласны с пользовательским соглашением

http://www.mathnet.ru/rus/agreement

Параметры загрузки:

IP: 3.91 .87 .62

26 апреля 2023 г., 17:08:37 


\title{
О РАЗРУШЕНИИ РЕШЕНИЙ ЗАДАЧ КОШИ ДЛЯ ОДНОГО КЛАССА НЕЛИНЕЙНЫХ УРАВНЕНИЙ ТЕОРИИ ФЕРРИТОВ
}

\author{
(c) 2020 г. $\quad$ М. О. КОРПУСОВ, г. И. ШЛЯПУГИН
}

\begin{abstract}
АннотАция. В работе рассмотрены три нелинейных уравнения теории магнетиков с градиентными нелинейностями $|\nabla u|^{q}, \partial_{t}|\nabla u|^{q}$ и $\partial_{t}^{2}|\nabla u|^{q}$. Для соответствующих задач Коши получены результаты о локальной во времени однозначной разрешимости в слабом смысле и о разрушении за конечное время. Эти три уравнения объединены наличием критического показателя $q=3 / 2$, поскольку поведение слабых решений различно при $1<q \leqslant 3 / 2$ и при $q>3 / 2$. Методом нелинейной емкости С. И. Похожаева получены априорные оценки, из которых и вытекают результаты об отсутствии локальных и глобальных слабых решений.
\end{abstract}

Ключевые слова: нелинейное уравнение соболевского типа, разрушение, локальная разрешимость, нелинейная емкость, оценка времени разрушения.

\section{ON BLOW-UP OF SOLUTIONS OF THE CAUCHY PROBLEMS FOR A CLASS OF NONLINEAR EQUATIONS OF FERRITE THEORY}

\author{
(c) 2020 M. O. KORPUSOV, G. I. SHLYAPUGIN
}

\begin{abstract}
In this paper, we consider three nonlinear equations of the theory of magnets with gradient nonlinearities $|\nabla u|^{q}, \partial_{t}|\nabla u|^{q}$, and $\partial_{t}^{2}|\nabla u|^{q}$ are considered. For the corresponding Cauchy problems, we obtain results on local-in-time unique solvability in the weak sense and on blow-up for a finite time. These three equations have the same critical exponent $q=3 / 2$ since weak solutions behave differently for $1<q \leqslant 3 / 2$ and for $q>3 / 2$. By the method of nonlinear capacity proposed by S. I. Pokhozhaev, we obtain a priori estimates, which imply the absence of local and global weak solutions.
\end{abstract}

Keywords and phrases: nonlinear Sobolev-type equation, blow-up, local solvability, nonlinear capacity, estimates of the blow-up time.

AMS Subject Classification: 35B44

\section{СОДЕРЖАНИЕ}

1. Введение . . . . . . . . . . . . . . . . . . . . . . . . . . 80

2. Обозначения . . . . . . . . . . . . . . . . . . . . . . . . 81

3. Фундаментальное решение . . . . . . . . . . . . . . . . . . . . . . . 83

4. Вторая формула Грина . . . . . . . . . . . . . . . . . . . . . . . . . . . . . . . 86

5. Третья формула Грина в ограниченной области . . . . . . . . . . . . . . . . . . . 87

6. Третья формула Грина во всем пространстве . . . . . . . . . . . . . . . . . . . . . . . . 92

7. Слабые решения задач Коши . . . . . . . . . . . . . . . . . . . . . . . . . . . . . . . . . 94

8. Свойства объемного и поверхностных потенциалов . . . . . . . . . . . . . . . . . . . . 99 
9. Задача Коши (7.1), (7.2): существование локальных и отсутствие локальных и глобальных во времени слабых решений . . . . . . . . . . . . . . . . . . . . . . . . . . 112

10. Задача Коши (7.3), (7.4): существование локальных и отсутствие локальных и глобальных во времени слабых решений . . . . . . . . . . . . . . . . . . . . . . . . . . 119

11. Задача Коши (7.5), (7.6): существование локальных и отсутствие локальных и глобальных во времени слабых решений . . . . . . . . . . . . . . . . . . . . . . . . . . 125

12. Приложение . . . . . . . . . . . . . . . . . . . . . . . . . . . . . . . . 128

Список литературы . . . . . . . . . . . . . . . . . . . . . . . . . . . . . . . . . 130

\section{1. ВВЕДЕНИЕ}

В [11] были получены линейные уравнения волн намагниченности в ферритах типа «легкая ось» и «легкая плоскость» (см. [11, уравнения (4.12), (4.18)]), которые можно записать в следующем виде:

$$
\frac{\partial^{2}}{\partial t^{2}} \Delta \psi+\omega_{1}^{2} \psi_{x_{1} x_{1}}+\omega_{2}^{2} \psi_{x_{2} x_{2}}+\omega_{3}^{2} \psi_{x_{3} x_{3}}=f, \quad \psi=\psi(x, t), \quad f=f(x, t)
$$

при $(x, t) \in \mathbb{R}^{3} \otimes[0, T]$, где $\omega_{1}, \omega_{2}, \omega_{3}$ - некоторые частоты (вообще говоря, попарно различные), а функция $\psi(x, t)$ - магнитный потенциал. Функция $f(x, t)$ в правой части задана явно. В данной работе мы рассмотрим следующие три нелинейных уравнения с модельными нелинейностями:

$$
\begin{gathered}
\frac{\partial^{2}}{\partial t^{2}} \Delta \psi+\omega_{1}^{2} \psi_{x_{1} x_{1}}+\omega_{2}^{2} \psi_{x_{2} x_{2}}+\omega_{3}^{2} \psi_{x_{3} x_{3}}+|\nabla \psi|^{q}=0 \\
\frac{\partial^{2}}{\partial t^{2}} \Delta \psi+\omega_{1}^{2} \psi_{x_{1} x_{1}}+\omega_{2}^{2} \psi_{x_{2} x_{2}}+\omega_{3}^{2} \psi_{x_{3} x_{3}}-\frac{\partial|\nabla \psi|^{q}}{\partial t}=0 \\
\frac{\partial^{2}}{\partial t^{2}} \Delta \psi+\omega_{1}^{2} \psi_{x_{1} x_{1}}+\omega_{2}^{2} \psi_{x_{2} x_{2}}+\omega_{3}^{2} \psi_{x_{3} x_{3}}+\frac{\partial^{2}|\nabla \psi|^{q}}{\partial t^{2}}=0
\end{gathered}
$$

при $q>1,(x, t) \in \mathbb{R}^{3} \otimes[0, T]$ и $\psi=\psi(x, t)$. Данная работа продолжает исследования, начатые в [8-10,17] и посвященные нахождению критических показателей для уравнений соболевского типа.

Уравнения (1.2)-(1.4) относятся к классу нелинейных уравнений типа С. Л. Соболева. Отметим, что исследованию линейных и нелинейных уравнений соболевского типа посвящено много работ. Так, в работах Г. А. Свиридюка, С. А. Загребиной, А. А. Замышляевой (см. [6, 15, 18]) были рассмотрены в общем виде и в виде примеров начально-краевые задачи для большого многообразия классов линейных и нелинейных уравнений соболевского типа.

Отметим, что впервые теория потенциала для неклассических уравнений типа С. Л. Соболева была рассмотрена в работе Б. В. Капитонова [7]. В дальнейшем теория потенциала изучалась в работах С. А. Габова и А. Г. Свешникова (см. [2,3]), а также в работах их учеников (см. работу Ю. Д. Плетнера [13]).

В классической работе [14] С. И. Похожаева и Э. Митидиери достаточно простым методом нелинейной емкости были получены глубокие результаты о роли так называемых критических показателей. Отметим также работы $[4,16]$ Е. И. Галахова и О. А. Салиевой.

В настоящей работе мы получили результат о существовании такого критического показателя $q=3 / 2$, что поведение слабых решений задач Коши для уравнения (1.2) при $1<q \leqslant 3 / 2$ и $q>3 / 2$ существенно различно. При $1<q \leqslant 3 / 2$ локальные во времени слабые решения отсутствуют в широком классе начальных функций, а при $q>3 / 2$ уже существуют. Для решений задачи Коши для уравнений (1.3) и (1.4) мы докажем отсутствие глобальных и локальных во времени решений в некоторых классах начальных функций при $1<q \leqslant 3 / 2$. 


\section{2. ОБОЗНАЧЕНИЯ}

В дальнейшем мы будем систематически использовать абстрактные банаховы пространства вида $\mathbb{C}^{(n)}([0, T] ; \mathbb{B})$, где $\mathbb{B}$ - банахово пространство относительно нормы $|\cdot|_{\mathbb{B}}, n \in \mathbb{Z}_{+}$. Определяется это пространство индуктивно. Сначала определим банахово пространство $\mathbb{C}([0, T] ; \mathbb{B})$ как линейное пространство таких функций $f(t):[0, T] \rightarrow \mathbb{B}$, что

$$
\left|f\left(t_{2}\right)-f\left(t_{1}\right)\right|_{\mathbb{B}} \rightarrow+0 \quad \text { для любых } t_{1}, t_{2} \in[0, T],\left|t_{2}-t_{1}\right| \rightarrow+0 .
$$

Линейное пространство $\mathbb{C}([0, T] ; \mathbb{B})$ является банаховым относительно нормы

$$
\|f(t)\|_{T}:=\sup _{t \in[0, T]}|f(t)|_{\mathbb{B}} .
$$

Функция $f(t)$ лежит в классе $\mathbb{C}^{(1)}([0, T] ; \mathbb{B})$, если $f(t) \in \mathbb{C}([0, T] ; \mathbb{B})$ и существует сильная производная $d f(t) / d t \in \mathbb{C}([0, T] ; \mathbb{B})$, определяемая следующим образом:

$$
\lim _{|\Delta t| \rightarrow+0}\left|\frac{1}{\Delta t}[f(t+\Delta t)-f(t)]-\frac{d f}{d t}(t)\right|_{\mathbb{B}}=0 .
$$

Далее индуктивно определяем банахово пространство $\mathbb{C}^{(n)}([0, T] ; \mathbb{B})$ для произвольного $n \in \mathbb{N}$.

В качестве банахова пространства $\mathbb{B}$ будут выступать следующие банаховы пространства: пространство $\mathbb{C}_{b}\left(\mathbb{R}^{3}\right)$ относительно нормы

$$
|f(x)|_{0}:=\sup _{x \in \mathbb{R}^{3}}|f(x)|,
$$

пространство $\mathbb{C}_{b}\left(\rho(x) ; \mathbb{R}^{3}\right)$ при $\rho(x) \geqslant \rho_{0}>0$ для всех $x \in \mathbb{R}^{3}$, которое является банаховым относительно нормы (более подробно см. доказательство леммы 2.1)

$$
|f(x)|_{0, \rho}:=\sup _{x \in \mathbb{R}^{3}} \rho(x)|f(x)|,
$$

а также пространство $\mathbb{C}_{b}^{\gamma_{1}, \gamma_{2}}\left(\rho(x) ; \mathbb{R}^{3}\right)$, где $\rho(x) \geqslant \rho_{0}>0$ для всех $x \in \mathbb{R}^{3}$ и $\gamma_{1}>0, \gamma_{2}>0$, которое определяется как линейное подпространство банахова пространства $\mathbb{C}_{b}^{(1)}\left(\mathbb{R}^{3}\right) \ni f(x)$, в котором конечна норма

$$
|f(x)|_{\gamma_{1}, \gamma_{2}}:=\sup _{x \in \mathbb{R}^{3}} \rho^{\gamma_{2}}(x)|f(x)|+\sum_{j=1}^{3} \sup _{x \in \mathbb{R}^{3}} \rho^{\gamma_{1}}(x)\left|\frac{\partial f(x)}{\partial x_{j}}\right| .
$$

Лемма 2.1. Линейное пространство $\mathbb{C}_{b}^{\gamma_{1}, \gamma_{2}}\left(\rho(x) ; \mathbb{R}^{3}\right)$ является банаховым относительно нормй (2.3).

Доказательство. Пусть $\left\{f_{n}(x)\right\} \subset \mathbb{C}_{b}^{\gamma_{1}, \gamma_{2}}\left(\rho(x) ; \mathbb{R}^{3}\right)$ - фундаментальная последовательность, т.е.

$$
\left|f_{n}-f_{m}\right|_{\gamma_{1}, \gamma_{2}} \rightarrow+0 \quad \text { при } n, m \rightarrow+\infty .
$$

Тогда, в частности, справедливы следующие соотношения:

$$
\left.|| f_{n}\right|_{\gamma_{1}, \gamma_{2}}-\left|f_{m}\right|_{\gamma_{1}, \gamma_{2}}|\leqslant| f_{n}-\left.f_{m}\right|_{\gamma_{1}, \gamma_{2}} \rightarrow+0 \quad \text { при } n, m \rightarrow+\infty .
$$

Таким образом, числовая последовательность $\left\{\left|f_{n}\right|_{\gamma_{1}, \gamma_{2}}\right\}$ является фундаментальной, а следовательно, сходится, и поэтому эта последовательность ограниченна:

$$
\left|f_{n}\right|_{\gamma_{1}, \gamma_{2}} \leqslant K<+\infty
$$

где $K>0$ - постоянная, не зависящая от $n \in \mathbb{N}$. Заметим, что имеет место неравенство

$$
|f|_{1}^{\prime}:=\min \left\{\rho_{0}^{\gamma_{1}}, \rho_{0}^{\gamma_{2}}\right\}\left[|f|_{0}+\sum_{j=1}^{3}\left|\frac{\partial f(x)}{\partial x_{j}}\right|_{0}\right] \leqslant\left|\rho^{\gamma_{2}}(x) f(x)\right|_{0}+\sum_{j=1}^{3}\left|\rho^{\gamma_{1}}(x) \frac{\partial f(x)}{\partial x_{j}}\right|_{0}=:|f|_{\gamma_{1}, \gamma_{2}},
$$

где $|g|_{0}:=\sup _{x \in \mathbb{R}^{3}}|g(x)|$. Из (2.4) и (2.7) вытекает, что последовательность $\left\{f_{n}\right\}$ является фундаментальной последовательностью в банаховом пространстве $\mathbb{C}_{b}^{(1)}\left(\mathbb{R}^{3}\right)$ относительно нормы $\mid \cdot l_{1}^{\prime}$, 
определенной равенством из (2.7). Следовательно, существует такая функция $f_{0}(x) \in \mathbb{C}_{b}^{(1)}\left(\mathbb{R}^{3}\right)$, что

$$
\left|f_{n}-f_{0}\right|_{1}^{\prime} \rightarrow+0 \text { при } n \rightarrow+\infty \text {. }
$$

Из (2.4) вытекает, что для любого $\varepsilon>0$ найдется такое $N \in \mathbb{N}$, что для всех $n, m \geqslant N$ будет выполнено неравенство

$$
\rho^{\gamma_{2}}(x)\left|f_{n}(x)-f_{m}(x)\right|+\rho^{\gamma_{1}}(x) \sum_{j=1}^{3}\left|\frac{\partial f_{n}(x)}{\partial x_{j}}-\frac{\partial f_{m}(x)}{\partial x_{j}}\right| \leqslant\left|f_{n}-f_{m}\right|_{\gamma_{1}, \gamma_{2}} \leqslant \varepsilon .
$$

Перейдя в этом поточечном неравенстве к пределу при $m \rightarrow+\infty$, получим следующее неравенство:

$$
\rho^{\gamma_{2}}(x)\left|f_{n}(x)-f_{0}(x)\right|+\rho^{\gamma_{1}}(x) \sum_{j=1}^{3}\left|\frac{\partial f_{n}(x)}{\partial x_{j}}-\frac{\partial f_{0}(x)}{\partial x_{j}}\right| \leqslant \varepsilon .
$$

В силу (2.6) и (2.10) приходим к следующим неравенствам:

$$
\begin{gathered}
\left|f_{n}-f_{0}\right|_{\gamma_{1}, \gamma_{2}} \leqslant \varepsilon \\
\left|f_{0}\right|_{\gamma_{1}, \gamma_{2}} \leqslant\left|f_{n}-f_{0}\right|_{\gamma_{1}, \gamma_{2}}+\left|f_{n}\right|_{\gamma_{1}, \gamma_{2}} \leqslant \varepsilon+K<+\infty .
\end{gathered}
$$

Таким образом, из $(2.11)$ и (2.12) вытекает, что фундаментальная последовательность $\left\{f_{n}\right\}$ сходится сильно к некоторой функции $f_{0}(x) \in \mathbb{C}_{b}^{\gamma_{1}, \gamma_{2}}\left(\rho(x) ; \mathbb{R}^{3}\right)$ по норме $(2.3)$.

Важными для нас будут также следующие банаховы пространства. Во-первых, пространство

$$
\mathbb{C}_{b}^{1,0}\left(\left(1+|x|^{2}\right)^{1 / 2} ; \mathbb{R}^{3}\right)=\mathbb{C}_{b}^{\gamma_{1}, \gamma_{2}}\left(\rho(x) ; \mathbb{R}^{3}\right)
$$

при $\rho(x)=\left(1+|x|^{2}\right)^{1 / 2}$ и $\gamma_{1}=1$ и $\gamma_{2}=0$, норму которого будем обозначать символом $|\cdot|_{1}$ :

$$
|f(x)|_{1}:=|f(x)|_{0}+\sum_{j=1}^{3}\left|\left(1+|x|^{2}\right)^{1 / 2} \frac{\partial f(x)}{\partial x_{j}}\right|_{0} .
$$

Во-вторых, будем также использовать банахово пространство

$$
\mathbb{C}_{b}^{1,1 / 2}\left(\left(1+|x|^{2}\right) ; \mathbb{R}^{3}\right)=\mathbb{C}_{b}^{\gamma_{1}, \gamma_{2}}\left(\rho(x) ; \mathbb{R}^{3}\right)
$$

при $\rho(x)=1+|x|^{2}, \gamma_{1}=1$ и $\gamma_{2}=1 / 2$. Далее, будет встречаться банахово пространство $\mathbb{C}_{b}^{(2)}\left(\left(1+|x|^{2}\right)^{\beta} ; \mathbb{R}^{3}\right)$ при $\beta>0$, которое является линейным подпространством банахова пространства $\mathbb{C}_{b}^{(2)}\left(\mathbb{R}^{3}\right) \ni f(x)$, в котором конечна норма

$$
|f|_{2}:=\sup _{x \in \mathbb{R}^{3}}\left(1+|x|^{2}\right)^{1 / 2}|f(x)|+\sum_{j=1}^{3} \sup _{x \in \mathbb{R}^{3}}\left(1+|x|^{2}\right)\left|\frac{\partial f(x)}{\partial x_{j}}\right|+\sum_{j, k=1,1}^{3,3} \sup _{x \in \mathbb{R}^{3}}\left(1+|x|^{2}\right)^{\beta}\left|\frac{\partial^{2} f(x)}{\partial x_{j} \partial x_{k}}\right| .
$$

Символом $\mathbb{C}_{0}^{(2)}[0, T]$ обозначаем банахово пространство функций из $\mathbb{C}^{(2)}[0, T] \ni f(t)$, для которых $f(T)=f^{\prime}(T)=f^{\prime \prime}(T)=0$, относительно стандартной нормы

$$
\|f(t)\|:=|f(t)|_{0}+\left|f^{\prime}(t)\right|_{0}+\left|f^{\prime \prime}(t)\right|_{0},
$$

где норма $|\cdot|_{0}$ определяется формулой (2.1). Будем использовать следующие обозначения для норм специальных банаховых пространств:

$$
\begin{array}{ll}
\mathbb{C}\left([0, T] ; \mathbb{C}_{b}^{1,0}\left(\left(1+|x|^{2}\right)^{1 / 2} ; \mathbb{R}^{3}\right)\right): & \|f\|_{0, T}:=\sup _{t \in[0, T]}|f(x, t)|_{1}, \\
\mathbb{C}^{(1)}\left([0, T] ; \mathbb{C}_{b}^{1,0}\left(\left(1+|x|^{2}\right)^{1 / 2} ; \mathbb{R}^{3}\right)\right): & \|f\|_{1, T}:=\sup _{t \in[0, T]}\left[|f(x, t)|_{1}+\left|\frac{\partial f(t)}{\partial t}\right|_{1}\right], \\
\mathbb{C}^{(2)}\left([0, T] ; \mathbb{C}_{b}^{1,0}\left(\left(1+|x|^{2}\right)^{1 / 2} ; \mathbb{R}^{3}\right)\right): & \|f\|_{2, T}:=\sup _{t \in[0, T]}\left[|f(x, t)|_{1}+\left|\frac{\partial f(t)}{\partial t}\right|_{1}+\left|\frac{\partial^{2} f(t)}{\partial t^{2}}\right|_{1}\right],
\end{array}
$$


где норма $|\cdot|_{1}$ определена равенством (2.14). Символом $\mathbb{C}_{x, t}^{(0, n)}\left(\mathbb{R}^{3} \otimes[0, T]\right)$, где $n \in \mathbb{N} \cup\{0\}$, обозначаем пространство таких функций $f(x, t)$, что

$$
f(x, t), \quad \frac{\partial^{j} f(x, t)}{\partial t^{j}} \in \mathbb{C}\left(\mathbb{R}^{3} \otimes[0, T]\right) \quad \text { при } j=\overline{0, n} .
$$

Символами $\mathscr{D}\left(\mathbb{R}^{3}\right), \mathscr{D}(-\infty,+\infty), \mathscr{D}(-\infty, T), \mathscr{D}\left(\mathbb{R}^{3} \otimes(-\infty, T)\right)$ и $\mathscr{D}\left(\mathbb{R}^{3} \otimes(-\infty,+\infty)\right)$ обозначаем векторные топологические пространства основных функций с компактными носителями из соответствующих множеств, а через $\mathscr{D}^{\prime}\left(\mathbb{R}^{3}\right), \mathscr{D}^{\prime}(-\infty,+\infty), \mathscr{D}^{\prime}(-\infty, T), \mathscr{D}^{\prime}\left(\mathbb{R}^{3} \otimes(-\infty, T)\right)$ и $\mathscr{D}^{\prime}\left(\mathbb{R}^{3} \otimes(-\infty,+\infty)\right)$ - соответствующие пространства обобщенных функций.

Символ $W_{q, \mathrm{loc}}^{1,0}\left(\mathbb{R}^{3} \otimes[0, T]\right)$ обозначает пространство измеримых функций $f(x, t)$, для которых определены слабые частные производные, причем для любого компакта $K \in \mathbb{R}^{3}$ имеем

$$
f(x, t), \frac{\partial f(x, t)}{\partial x_{j}} \in L^{q}(K \otimes[0, T]) .
$$

Символ $W_{q, \mathrm{loc}}^{1,0}\left(\mathbb{R}^{3} \otimes[0,+\infty)\right)$ обозначает пространство измеримых функций $f(x, t)$, для которых определены слабые частные производные, причем для любого компакта $K \in \mathbb{R}^{3}$ и для любого $T>0$ имеем

$$
f(x, t), \frac{\partial f(x, t)}{\partial x_{j}} \in L^{q}(K \otimes[0, T]) .
$$

Символ $W_{q, \text { loc }}^{1,0}\left(\mathbb{R}^{3}\right)$ обозначает пространство таких измеримых функций $f(x)$, что существуют все слабые производные и для любого компакта $K \subset \mathbb{R}^{3}$ имеем

$$
f(x), \frac{\partial f(x)}{\partial x_{j}} \in L^{q}(K) .
$$

Символами $W_{q}^{1}(D)$ и $H^{2}\left(O\left(x_{0}, R_{0}\right)\right)$ будем обозначать классические пространства С. Л. Соболева, где $O\left(x_{0}, R_{0}\right)$ - открытый шар радиуса $R_{0}>0$ в $\mathbb{R}^{3}$ с центром в точке $x_{0} \in \mathbb{R}^{3}$.

Наконец, для функции $f(t) \in \mathbb{C}^{(n)}(-\infty, 0] \cup \mathbb{C}^{(n)}[0,+\infty)$, где $n \in \mathbb{N}$ и производные в точке $t=0$ понимаются в смысле односторонних пределов, будем использовать следующее обозначение:

$$
\left\{\frac{\partial^{j} f(t)}{\partial t^{j}}\right\}= \begin{cases}f^{(j)}(t), & \text { если }\{t>0\} \cup\{t<0\}, \quad j=\overline{1, n} . \\ \text { произвольно, } & \text { если } t=0,\end{cases}
$$

Пусть $f(t)$ - некоторая функция, определенная при $t \in[0, T]$; тогда символом $\widetilde{f}(t)$ будем обозначать продолжение нулем при $t<0$ :

$$
\widetilde{f}(t)= \begin{cases}f(t), & \text { если } t \in[0, T] \\ 0, & \text { если } t<0\end{cases}
$$

\section{3. ФУНДАМЕНТАЛЬНОЕ РЕШЕНИЕ}

Построим фундаментальное решение оператора

$$
\mathfrak{M}_{x, t}[u](x, t):=\frac{\partial^{2}}{\partial t^{2}} \Delta u(x, t)+\omega_{1}^{2} u_{x_{1} x_{1}}(x, t)+\omega_{2}^{2} u_{x_{2} x_{2}}(x, t)+\omega_{3}^{2} u_{x_{3} x_{3}}(x, t),
$$

где, вообще говоря, $\omega_{1}>0, \omega_{2}>0, \omega_{3}>0$ попарно различны. Рассмотрим следующее уравнение в смысле пространства $\mathscr{D}^{\prime}\left(\mathbb{R}^{4}\right)$ :

$$
\mathfrak{M}_{x, t}[\mathscr{E}](x, t)=\delta(x) \delta(t)
$$

Применяя преобразование Лапласа, получим следующее уравнение:

$$
\left(p^{2}+\omega_{1}^{2}\right) \overline{\mathscr{E}}_{x_{1} x_{1}}(x, p)+\left(p^{2}+\omega_{2}^{2}\right) \overline{\mathscr{E}}_{x_{2} x_{2}}(x, p)+\left(p^{2}+\omega_{3}^{2}\right) \overline{\mathscr{E}}_{x_{3} x_{3}}(x, p)=\delta\left(x_{1}\right) \delta\left(x_{2}\right) \delta\left(x_{3}\right) .
$$

Одним из решений этого уравнения является функция

$$
\overline{\mathscr{E}}(x, p)=-\frac{1}{4 \pi} \frac{1}{\sqrt{\left(p^{2}+\omega_{2}^{2}\right)\left(p^{2}+\omega_{3}^{3}\right) x_{1}^{2}+\left(p^{2}+\omega_{1}^{2}\right)\left(p^{2}+\omega_{3}^{3}\right) x_{2}^{2}+\left(p^{2}+\omega_{1}^{2}\right)\left(p^{2}+\omega_{2}^{3}\right) x_{3}^{2}}} .
$$


Последнее выражение можно преобразовать к виду

$$
\overline{\mathscr{E}}(x, p)=-\frac{1}{4 \pi|x|} \frac{1}{p^{2}}\left[1+\frac{\alpha_{1}(x)}{p^{2}}+\frac{\alpha_{2}(x)}{p^{4}}\right]^{-1 / 2},
$$

где

$$
\begin{aligned}
\alpha_{1}(x) & :=\frac{\left(\omega_{2}^{2}+\omega_{3}^{2}\right) x_{1}^{2}+\left(\omega_{1}^{2}+\omega_{3}^{2}\right) x_{2}^{2}+\left(\omega_{1}^{2}+\omega_{2}^{2}\right) x_{3}^{2}}{x_{1}^{2}+x_{2}^{2}+x_{3}^{2}}, \\
\alpha_{2}(x) & :=\frac{\omega_{2}^{2} \omega_{3}^{2} x_{1}^{2}+\omega_{1}^{2} \omega_{3}^{2} x_{2}^{2}+\omega_{1}^{2} \omega_{2}^{2} x_{3}^{2}}{x_{1}^{2}+x_{2}^{2}+x_{3}^{2}} .
\end{aligned}
$$

Предположим, что $\operatorname{Re} p=\sigma>R$ при достаточно большом $R>0$. Тогда $|p|>R$, и поэтому справедливо следующее разложение в ряд:

$$
\begin{aligned}
\frac{1}{p^{2}}\left[1+\frac{\alpha_{1}(x)}{p^{2}}+\frac{\alpha_{2}(x)}{p^{4}}\right]^{-1 / 2} & =\sum_{k=0}^{+\infty}\left(\begin{array}{c}
-1 / 2 \\
k
\end{array}\right) \frac{1}{p^{2 k+2}}\left(\alpha_{1}(x)+\frac{\alpha_{2}(x)}{p^{2}}\right)^{k}=\frac{1}{p^{2}}+\bar{\Phi}(x, p), \\
\bar{\Phi}(x, p) & =\sum_{k=1}^{+\infty}\left(\begin{array}{c}
-1 / 2 \\
k
\end{array}\right) \frac{1}{p^{2 k+2}}\left(\alpha_{1}(x)+\frac{\alpha_{2}(x)}{p^{2}}\right)^{k} .
\end{aligned}
$$

Поскольку $|p| \geqslant R>0$, то применяя обратное преобразование Лапласа к функции $\bar{\Phi}(x, p)$, получим выражение

$$
\Phi(x, t)=\theta(t) \sum_{k=1}^{+\infty}\left(\begin{array}{c}
-1 / 2 \\
k
\end{array}\right) \frac{1}{(2 k+1) !}\left(\alpha_{1}(x) \mathrm{id}+\alpha_{2}(x) t *\right)^{k} t^{2 k+1}
$$

где

$$
\left(\alpha_{1}(x) \mathrm{id}+\alpha_{2}(x) t *\right) \phi(t):=\alpha_{1}(x) \phi(t)+\alpha_{2}(x) \int_{0}^{t}(t-\tau) \phi(\tau) d \tau .
$$

Таким образом, для фундаментального решения справедливо равенство

$$
\mathscr{E}(x, t)=\frac{1}{2 \pi i} \int_{\sigma-i \infty}^{\sigma+i \infty} \overline{\mathscr{E}}(x, p) e^{p t} d p, \quad \sigma>R>0
$$

и с учетом равенства (3.10) имеем

$$
\mathscr{E}(x, t)=-\frac{t \theta(t)}{4 \pi|x|}-\frac{1}{4 \pi|x|} \Phi(x, t),
$$

где функция $\Phi(x, t)$ определена равенством (3.10). Фундаментальное решение обладает свойствами, которые мы сформулируем в виде следующей теоремы.

Теорема 3.1. Фундаментальное решение $\mathscr{E}(x, t)$ обладает следующими свойствами:

1. $\mathscr{E}(x, t) \in \mathbb{C}^{(\infty)}\left(\mathbb{R}^{3} \backslash\{0\} \otimes[0,+\infty)\right)$.

2. При $x \neq(0,0,0)$ u $t \in[0, T]$ справедливы следующие оценки:

$$
\begin{gathered}
\left|\frac{\partial^{k} \mathscr{E}}{\partial t^{k}}(x, t)\right| \leqslant \frac{K_{1}(T)}{|x|}, \quad\left|\frac{\partial^{k+1} \mathscr{E}}{\partial t^{k} \partial x_{j}}(x, t)\right| \leqslant \frac{K_{2}(T)}{|x|^{2}}, \\
\left|\frac{\partial^{k+2} \mathscr{E}}{\partial t^{k} \partial x_{j} \partial x_{i}}(x, t)\right| \leqslant \frac{K_{3}(T)}{|x|^{3}},
\end{gathered}
$$

где все смешанные производные перестановочны и $k \in \mathbb{N} \cup\{0\}, i, j=1,2,3$.

3. При $x \neq(0,0,0)$ u $t \geqslant 0$ справедливы следующие равенства:

$$
\frac{\partial^{l} \mathscr{E}}{\partial x_{j}^{l}}(x, 0)=0, \quad \frac{\partial^{l}}{\partial x_{j}^{l}} \frac{\partial \mathscr{E}}{\partial t}(x, 0)=-\frac{1}{4 \pi} \frac{\partial^{l}}{\partial x_{j}^{l}} \frac{1}{|x|}, \quad l=0,1 .
$$


4. При $x \neq(0,0,0)$ u $t \geqslant 0$ справедливо равенство

$$
\frac{\partial^{2+k} \mathscr{E}}{\partial t^{2} \partial x_{j}^{k}}(x, 0)=0, \quad k=0,1
$$

где все смешанные производные перестановочны.

Доказательство. Свойство 1 немедленно следует из явного вида (3.12), из которого видна бесконечная дифференцируемость при $x \neq(0,0,0)$ и $t \in[0, T]$.

Для доказательства свойства 2 прежде всего заметим, что фундаментальное решение при $x \neq(0,0,0)$ и $t \in[0, T]$ бесконечно дифференцируемо по $t \in[0, T]$ и все производные имеют особенность по $x \in \mathbb{R}^{3}$ вида

$$
\frac{\Psi(x, t)}{|x|}, \quad|\Psi(x, t)| \leqslant K_{1}(T)<+\infty, \quad t \in[0, T] .
$$

Теперь заметим, что

$$
\begin{gathered}
\frac{\partial \mathscr{E}}{\partial x_{j}}(x, t)=\frac{t}{4 \pi} \frac{x_{j}}{|x|^{3}}+\frac{\Phi(x, t)}{4 \pi} \frac{x_{j}}{|x|^{3}}-\frac{1}{4 \pi|x|} \frac{\partial \Phi(x, t)}{\partial x_{j}}, \\
\frac{\partial \Phi(x, t)}{\partial x_{j}}=\sum_{k=1}^{+\infty}\left(\begin{array}{c}
-1 / 2 \\
k
\end{array}\right) \frac{k}{(2 k+1) !}\left(\alpha_{1}(x) \mathrm{id}+\alpha_{2}(x) t *\right)^{k-1}\left(\frac{\partial \alpha_{1}(x)}{\partial x_{j}} \mathrm{id}+\frac{\partial \alpha_{2}(x)}{\partial x_{j}} t *\right) t^{2 k+1}, \\
0 \leqslant \alpha_{1}(x) \leqslant \omega_{1}^{2}+\omega_{2}^{2}+\omega_{3}^{2}, \quad 0 \leqslant \alpha_{2}(x) \leqslant \omega_{1}^{2} \omega_{2}^{2}+\omega_{2}^{2} \omega_{3}^{2}+\omega_{3}^{2} \omega_{1}^{2}, \\
\left|\frac{\partial \alpha_{1}(x)}{\partial x_{j}}\right| \leqslant \frac{c_{1}\left(\omega_{1}, \omega_{2}, \omega_{3}\right)}{|x|}, \quad\left|\frac{\partial \alpha_{2}(x)}{\partial x_{j}}\right| \leqslant \frac{c_{2}\left(\omega_{1}, \omega_{2}, \omega_{3}\right)}{|x|} .
\end{gathered}
$$

Таким образом, из (3.17)-(3.20) вытекает оценка

$$
\left|\frac{\partial \mathscr{E}(x, t)}{\partial x_{j}}\right| \leqslant \frac{K_{2}(T)}{|x|^{2}} .
$$

Аналогичным образом можно доказать оценку

$$
\left|\frac{\partial^{k+1} \mathscr{E}(x, t)}{\partial t^{k} \partial x_{j}}\right| \leqslant \frac{K_{2}(T)}{|x|^{2}}, \quad k \in \mathbb{N} .
$$

В силу свойства 1 все смешанные производные в левой части неравенства (3.22) перестановочны. Для доказательства неравенства (3.14) нужно установить оценки

$$
\left|\frac{\partial^{2} \alpha_{1}(x)}{\partial x_{j} \partial x_{i}}\right| \leqslant \frac{c_{3}\left(\omega_{1}, \omega_{2}, \omega_{3}\right)}{|x|^{2}}, \quad\left|\frac{\partial^{2} \alpha_{2}(x)}{\partial x_{j} \partial x_{i}}\right| \leqslant \frac{c_{4}\left(\omega_{1}, \omega_{2}, \omega_{3}\right)}{|x|^{2}} .
$$

Докажем свойство 3. Заметим, что, с одной стороны, при $x \neq(0,0,0)$ первое слагаемое

$$
J(x, t):=-\frac{t \theta(t)}{4 \pi|x|}
$$

в выражении (3.12) для фундаментального решения $\mathscr{E}(x, t)$ обладает при $t \geqslant 0$ следующими свойствами:

$$
\frac{\partial^{l}}{\partial x_{j}^{l}} J(x, 0)=0, \quad \frac{\partial^{l}}{\partial x_{j}^{l}} \frac{\partial J}{\partial t}(x, 0)=-\frac{1}{4 \pi} \frac{\partial}{\partial x_{j}} \frac{1}{|x|}, \quad l=0,1 .
$$

С другой стороны, в силу явного выражения (3.10) для функции $\Phi(x, t)$ при $x \neq(0,0,0)$ справедливы равенства

$$
\frac{\partial^{l}}{\partial x_{j}^{l}} \frac{\partial^{m}}{\partial t^{m}} \Phi(x, 0)=0, \quad l, m=0,1 .
$$

Поэтому из (3.24) и (3.25) вытекают равенства (3.15).

Свойство 4 является непосредственным следствием выражения (3.12) для фундаментального решения. 
В [10] были доказаны следующие оценки интегралов:

$$
\begin{aligned}
& \sup _{x \in \mathbb{R}^{3}} \int_{\mathbb{R}^{3}} \frac{\left(1+|x|^{2}\right)^{1 / 2}}{\left(1+|y|^{2}\right)^{\beta}} \frac{1}{|x-y|} d y=B_{1}(\beta)<+\infty, \quad \beta>\frac{3}{2}, \\
& \sup _{x \in \mathbb{R}^{3}} \int_{\mathbb{R}^{3}} \frac{1+|x|^{2}}{\left(1+|y|^{2}\right)^{\beta}} \frac{1}{|x-y|^{2}} d y=B_{2}(\beta)<+\infty, \quad \beta>\frac{3}{2} .
\end{aligned}
$$

Введем следующее обозначение:

$$
G_{\beta}(x, y, t):=\frac{\left(1+|x|^{2}\right)^{1 / 2}}{\left(1+|y|^{2}\right)^{\beta}} \mathscr{E}(x-y, t),
$$

где $\mathscr{E}(x, t)$ - фундаментальное решение, определенное формулой (3.11).

Теорема 3.2. Справедливы следующие оченки:

$$
\begin{gathered}
\sup _{(x, t) \in \mathbb{R}^{3} \otimes[0, T]} \int_{\mathbb{R}^{3}}\left|\frac{\partial^{k} G_{\beta}(x, y, t)}{\partial t^{k}}\right| d y=B_{3}(\beta, T)<+\infty, \\
\sup _{(x, t) \in \mathbb{R}^{3} \otimes[0, T]}\left(1+|x|^{2}\right)^{1 / 2} \int_{\mathbb{R}^{3}}\left|\frac{\partial^{k+1} G_{\beta}(x, y, t)}{\partial t^{k} \partial x_{j}}\right| d y=B_{4}(\beta, T)<+\infty, \quad j=1,2,3,
\end{gathered}
$$

при $\beta>3 / 2$ u $k=0,1,2,3$.

Доказательство теоремы 3.2 основано на применении оценок (3.13), (3.26) и (3.27).

\section{4. ВТОРАЯ ФОРМУЛА ГРИНА}

Пусть $u(x, t), v(x, t) \in \mathbb{C}^{(2)}\left([0, T] ; \mathbb{C}^{(2)}(\bar{\Omega})\right)$. Тогда справедливы следующие равенства:

$$
\begin{array}{r}
v \frac{\partial^{2}}{\partial t^{2}} \Delta u=\frac{\partial}{\partial t}\left(v \Delta \frac{\partial u}{\partial t}\right)-\frac{\partial}{\partial t}\left(\Delta u \frac{\partial v}{\partial t}\right)+\operatorname{div}\left(\frac{\partial^{2} v}{\partial t^{2}} \nabla u\right)-\operatorname{div}\left(u \nabla \frac{\partial^{2} v}{\partial t^{2}}\right)+u \Delta \frac{\partial^{2} v}{\partial t^{2}}= \\
=\frac{\partial}{\partial t}\left(v \Delta \frac{\partial u}{\partial t}\right)-\frac{\partial}{\partial t}\left(\Delta u \frac{\partial v}{\partial t}\right)+\operatorname{div}\left(v \nabla \frac{\partial^{2} u}{\partial t^{2}}\right)-\operatorname{div}\left(u \nabla \frac{\partial^{2} v}{\partial t^{2}}\right)+\frac{\partial}{\partial t} \operatorname{div}\left(\frac{\partial v}{\partial t} \nabla u\right)- \\
-\frac{\partial}{\partial t} \operatorname{div}\left(v \nabla \frac{\partial u}{\partial t}\right)+u \Delta \frac{\partial^{2} v}{\partial t^{2}}, \\
v \frac{\partial^{2} u}{\partial x_{j}^{2}}=\frac{\partial}{\partial x_{j}}\left(v \frac{\partial u}{\partial x_{j}}\right)-\frac{\partial}{\partial x_{j}}\left(u \frac{\partial v}{\partial x_{j}}\right)+u \frac{\partial^{2} v}{\partial x_{j}^{2}} \quad \text { при } \quad j=1,2,3 .
\end{array}
$$

Отметим, что в данном случае формально сопряженный оператор имеет следующий вид:

$$
\mathfrak{M}_{x, t}^{t}[u](x, t)=\mathfrak{M}_{x, t}[u](x, t) .
$$

Из равенств (4.1) и (4.2) вытекает вторая формула Грина:

$$
\begin{gathered}
\int_{0}^{t} \int_{\Omega}\left[v(\xi, \tau) \mathfrak{M}_{\xi, \tau}[u](\xi, \tau)-u(\xi, \tau) \mathfrak{M}_{\xi, \tau}^{t}[v](\xi, \tau)\right] d \xi d \tau= \\
=\int_{0}^{t} \int_{\partial \Omega}\left[v(\xi, \tau) \mathfrak{N}_{\xi, \tau}[u](\xi, \tau)-u(\xi, \tau) \mathfrak{N}^{t}[v](\xi, \tau)\right] d S_{\xi} d \tau+ \\
+\int_{\Omega}\left[v(\xi, t) \Delta \frac{\partial u}{\partial \tau}(\xi, t)-v(\xi, 0) \Delta \frac{\partial u}{\partial \tau}(\xi, 0)\right] d \xi-\int_{\Omega}\left[\frac{\partial v}{\partial \tau}(\xi, t) \Delta u(\xi, t)-\frac{\partial v}{\partial \tau}(\xi, 0) \Delta u(\xi, 0)\right] d \xi+
\end{gathered}
$$




$$
+\int_{\partial \Omega}\left[\frac{\partial v}{\partial \tau}(\xi, t) \frac{\partial u}{\partial n_{\xi}}(\xi, t)-\frac{\partial v}{\partial \tau}(\xi, 0) \frac{\partial u}{\partial n_{\xi}}(\xi, 0)\right] d S_{\xi}-\int_{\partial \Omega}\left[v(\xi, t) \frac{\partial^{2} u}{\partial \tau \partial n_{\xi}}(\xi, t)-v(\xi, 0) \frac{\partial^{2} u}{\partial \tau \partial n_{\xi}}(\xi, 0)\right] d S_{\xi}
$$

где введены обозначения

$$
\begin{aligned}
\mathfrak{N}_{\xi, \tau}[u](\xi, \tau)=\mathfrak{N}_{\xi, \tau}^{t}[u] & (\xi, \tau)= \\
= & \frac{\partial^{2}}{\partial \tau^{2}} \frac{\partial u}{\partial n_{\xi}}+\omega_{1}^{2} \frac{\partial u}{\partial \xi_{1}} \cos \left(n_{\xi}, e_{1}\right)+\omega_{2}^{2} \frac{\partial u}{\partial \xi_{2}} \cos \left(n_{\xi}, e_{2}\right)+\omega_{3}^{2} \frac{\partial u}{\partial \xi_{3}} \cos \left(n_{\xi}, e_{3}\right) .
\end{aligned}
$$

Сформулируем этот результат в виде теоремы.

Теорема 4.1. Для любых функций $u(x, t), v(x, t) \in \mathbb{C}^{(2)}\left([0, T] ; \mathbb{C}^{(2)}(\bar{\Omega})\right)$ nри $t \in[0, T]$ справедливо равенство (4.4).

\section{5. ТРЕТЬЯ ФОРМУЛА ГРИНА В ОГРАНИЧЕННОЙ ОБЛАСТИ}

Прежде всего докажем следующее утверждение.

Лемма 5.1. Пусть

$$
\begin{aligned}
f(x, t):=\int_{\partial O(x, 1)}\left[\frac{\partial^{2}}{\partial t^{2}} \frac{\partial \mathscr{E}}{\partial n_{\xi}}(x-\right. & \xi, t)+\omega_{1}^{2} \frac{\partial \mathscr{E}}{\partial \xi_{1}}(x-\xi, t) \cos \left(n_{\xi}, e_{1}\right)+ \\
& \left.+\omega_{2}^{2} \frac{\partial \mathscr{E}}{\partial \xi_{1}}(x-\xi, t) \cos \left(n_{\xi}, e_{2}\right)+\omega_{3}^{2} \frac{\partial \mathscr{E}}{\partial \xi_{3}}(x-\xi, t) \cos \left(n_{\xi}, e_{3}\right)\right] d S_{\xi},
\end{aligned}
$$

где $\mathscr{E}(x, t)$ - фундаментальное решение оператора $\mathfrak{M}_{x, t}$, определенное равенством (3.12). Тогда $f(x, t)=0$ для всех $t \geqslant 0$.

Доказательство. Для определенности будем считать, что $n_{\xi}$ - внутренняя нормаль в точке $\xi \in$ $\partial O(x, 1)$. Отметим, что в силу результатов п. 3 теоремы 3.1 справедливы следующие равенства:

$$
\mathscr{E}(x, 0)=0, \quad \frac{\partial \mathscr{E}(x, 0)}{\partial t}=-\frac{1}{4 \pi|x|}, \quad t \geqslant 0 .
$$

Если при этом $\xi \in \partial O(x, 1)$, то $\xi \neq x$ и потому

$$
\frac{\partial \mathscr{E}}{\partial n_{\xi}}(x-\xi, 0)=0, \quad \frac{\partial}{\partial t} \frac{\partial \mathscr{E}}{\partial n_{\xi}}(x-\xi, 0)=-\frac{1}{4 \pi} \frac{\partial}{\partial n_{\xi}} \frac{1}{|x-\xi|}, \quad t \geqslant 0 .
$$

Применяя преобразование Лапласа по $t$ к функции $f(x, t)$ и принимая во внимание (5.3), получим равенство

$$
\begin{array}{r}
\bar{f}(x, p)=\frac{1}{4 \pi} \int_{\partial O(x, 1)} \frac{\partial}{\partial n_{\xi}} \frac{1}{|x-\xi|} d S_{\xi}+\int_{\partial O(x, 1)}\left[p^{2} \frac{\partial \overline{\mathscr{E}}}{\partial n_{\xi}}(x-\xi, p)+\omega_{1}^{2} \frac{\partial \overline{\mathscr{E}}}{\partial \xi_{1}}(x-\xi, p) \cos \left(n_{\xi}, e_{1}\right)+\right. \\
\left.+\omega_{2}^{2} \frac{\partial \overline{\mathscr{E}}}{\partial \xi_{1}}(x-\xi, p) \cos \left(n_{\xi}, e_{2}\right)+\omega_{3}^{2} \frac{\partial \overline{\mathscr{E}}}{\partial \xi_{3}}(x-\xi, p) \cos \left(n_{\xi}, e_{3}\right)\right] d S_{\xi}
\end{array}
$$

Отметим, что в силу равенства (3.4) справедливы следующие формулы:

$$
\begin{aligned}
& \frac{\partial \overline{\mathscr{E}}}{\partial \xi_{1}}(z, p)=-\frac{1}{4 \pi} \frac{\left(p^{2}+\omega_{2}^{2}\right)\left(p^{2}+\omega_{3}^{2}\right) z_{1}}{\beta(z, p)}, \\
& \frac{\partial \overline{\mathscr{E}}}{\partial \xi_{2}}(z, p)=-\frac{1}{4 \pi} \frac{\left(p^{2}+\omega_{1}^{2}\right)\left(p^{2}+\omega_{3}^{2}\right) z_{2}}{\beta(z, p)}, \\
& \frac{\partial \overline{\mathscr{E}}}{\partial \xi_{3}}(z, p)=-\frac{1}{4 \pi} \frac{\left(p^{2}+\omega_{1}^{2}\right)\left(p^{2}+\omega_{2}^{2}\right) z_{3}}{\beta(z, p)},
\end{aligned}
$$


где $z_{j}=x_{j}-\xi_{j}, j=1,2,3$, и

$$
\beta(z, p):=\left(\left(p^{2}+\omega_{2}^{2}\right)\left(p^{2}+\omega_{3}^{3}\right) z_{1}^{2}+\left(p^{2}+\omega_{1}^{2}\right)\left(p^{2}+\omega_{3}^{3}\right) z_{2}^{2}+\left(p^{2}+\omega_{1}^{2}\right)\left(p^{2}+\omega_{2}^{3}\right) z_{3}^{2}\right)^{3 / 2} .
$$

Но тогда справедливо равенство

$$
\sum_{j=1}^{3}\left(p^{2}+\omega_{j}^{2}\right) \frac{\partial \overline{\mathscr{E}}(z, p)}{\partial \xi_{j}}=-\frac{1}{4 \pi}\left(p^{2}+\omega_{1}^{2}\right)\left(p^{2}+\omega_{2}^{2}\right)\left(p^{2}+\omega_{3}^{2}\right) \frac{|x-\xi|}{\beta(z, p)},
$$

где мы воспользовались тем, что

$$
\sum_{j=1}^{3}\left(x_{j}-\xi_{j}\right) \cos \left(n_{\xi}, e_{j}\right)=|x-\xi| .
$$

Заметим, что $|z|=|x-\xi|=1$ при $\xi \in \partial O(x, 1)$. Вычислим следующий интеграл:

$$
I(x, p):=-\int_{\partial O(x, 1)} \frac{1}{\beta(z, p)} d S_{\xi} .
$$

Перейдем к сферической системе координат с центром в точке $x$. Тогда интеграл $I(x, p)$ примет вид

$$
I(x, p):=-\int_{0}^{2 \pi} \int_{0}^{\pi} \frac{\sin \theta}{\left(\delta_{1}^{2} \sin ^{2} \theta+\delta_{2}^{2} \cos ^{2} \theta\right)^{3 / 2}} d \theta d \phi
$$

где

$$
\delta_{1}^{2}=\left(p^{2}+\omega_{3}^{2}\right)\left(\left(p^{2}+\omega_{2}^{2}\right) \cos ^{2} \phi+\left(p^{2}+\omega_{1}^{2}\right) \sin ^{2} \phi\right), \quad \delta_{2}^{2}=\left(p^{2}+\omega_{1}^{2}\right)\left(p^{2}+\omega_{2}^{2}\right) .
$$

Вычислим сначала внутренний интеграл в (5.12). Справедлива следующая цепочка равенств:

$$
I_{1}(p, \phi):=\int_{0}^{\pi} \frac{\sin \theta}{\left(\delta_{1}^{2} \sin ^{2} \theta+\delta_{2}^{2} \cos ^{2} \theta\right)^{3 / 2}} d \theta=\int_{-1}^{1} \frac{d t}{\left(\delta_{1}^{2}+\left(\delta_{2}^{2}-\delta_{1}^{2}\right) t^{2}\right)^{3 / 2}}=\frac{2}{\delta_{1}^{2} \delta_{2}},
$$

где использованы подстановка $t=-\cos \theta$ и формула 380.003 из [5]. Итак,

$$
\begin{aligned}
& I(x, p)=-\frac{2}{\left(p^{2}+\omega_{3}^{2}\right) \sqrt{\left(p^{2}+\omega_{1}^{2}\right)\left(p^{2}+\omega_{2}^{2}\right)}} \int_{0}^{2 \pi} \frac{1}{\left(p^{2}+\omega_{2}^{2}\right) \cos ^{2} \phi+\left(p^{2}+\omega_{1}^{2}\right) \sin ^{2} \phi} d \phi= \\
& =-\frac{2}{\left(p^{2}+\omega_{3}^{2}\right) \sqrt{\left(p^{2}+\omega_{1}^{2}\right)\left(p^{2}+\omega_{2}^{2}\right)}} \frac{2 \pi}{\sqrt{\left(p^{2}+\omega_{1}^{2}\right)\left(p^{2}+\omega_{2}^{2}\right)}}=-\frac{4 \pi}{\left(p^{2}+\omega_{1}^{2}\right)\left(p^{2}+\omega_{2}^{2}\right)\left(p^{2}+\omega_{3}^{2}\right)} .
\end{aligned}
$$

Из (5.4) получаем равенство

$$
\bar{f}(x, p)=\frac{1}{4 \pi} \int_{\partial O(x, 1)} \frac{\partial}{\partial n_{\xi}} \frac{1}{|x-\xi|} d S_{\xi}-1 .
$$

Заметим, что

$$
\begin{aligned}
& \int_{\partial O(x, 1)} \frac{\partial}{\partial n_{\xi}} \frac{1}{|x-\xi|} d S_{\xi}= \\
&=\int_{\partial O(x, 1)} \frac{\left(x_{1}-\xi_{1}\right) \cos \left(n_{\xi}, e_{1}\right)+\left(x_{2}-\xi_{2}\right) \cos \left(n_{\xi}, e_{2}\right)+\left(x_{3}-\xi_{3}\right) \cos \left(n_{\xi}, e_{3}\right)}{|x-\xi|^{3}} d S_{\xi}= \\
& \quad=\int_{\partial O(x, 1)} \frac{|x-\xi|}{|x-\xi|^{3}} d S_{\xi}=\int_{\partial O(x, 1)} d S_{\xi}=4 \pi .
\end{aligned}
$$


Из равенств (5.16) и (5.17) вытекает равенство $\bar{f}(x, p)=0$. Следовательно, $f(x, t)=0$ для всех $t \geqslant 0$. Лемма доказана.

Непосредственным следствием этой леммы является следующее утверждение.

Лемма 5.2. Для любого $\varepsilon>0$, для любого $x \in \mathbb{R}^{3} u$ любого $t>0$ справедливо следующее равенство:

$$
\int_{\partial O(x, \varepsilon)} \mathfrak{N}_{\xi, \tau}^{t}[\mathscr{E}(x-\xi, t-\tau)](\xi, \tau) d S_{\xi}=0, \quad \tau \in[0, t]
$$

Доказательство. Докажем утверждение для случая $\varepsilon \in(0,1)$. Отметим, что фундаментальное решение $\mathscr{E}(x-\xi, t-\tau)$ удовлетворяет уравнению

$$
\mathfrak{M}_{\xi, \tau}^{t}[\mathscr{E}(x-\xi, t-\tau)](\xi, \tau)=0, \quad \xi \neq x, \quad \tau \in[0, t] .
$$

Интегрируя равенство (5.19) по шаровому слою $O(x, 1) \backslash O(x, \varepsilon)$, получим равенство

$$
\int_{\partial O(x, \varepsilon)} \mathfrak{N}_{\xi, \tau}^{t}[\mathscr{E}(x-\xi, t-\tau)](\xi, \tau) d S_{\xi}=\int_{\partial O(x, 1)} \mathfrak{N}_{\xi, \tau}^{t}[\mathscr{E}(x-\xi, t-\tau)](\xi, \tau) d S_{\xi}=0,
$$

где последнее равенство справедливо в силу леммы 5.1 .

Пусть $x \in \Omega$ и $t \in(0, T]$. Теперь применим вторую формулу Грина (4.4) к функциям $u(\xi, \tau) \in \mathbb{C}^{(2)}\left([0, T] ; \mathbb{C}^{(2)}(\bar{\Omega})\right)$ и $v(\xi, \tau)=\mathscr{E}(x-\xi, t-\tau)$. Поскольку $x \in \Omega$, то найдется такое $\varepsilon \in(0,1)$, что $O(x, \varepsilon) \subset \Omega$. Поэтому $v(\xi, \tau)=\mathscr{E}(x-\xi, t-\tau) \in \mathbb{C}^{(2)}\left([0, T] ; \mathbb{C}^{(2)}(\bar{\Omega} \backslash O(x, \varepsilon))\right)$. Поэтому вторая формула Грина (4.4), примененная к указанным функциям в области $\Omega \backslash \overline{O(x, \varepsilon)}$, примет следующий вид:

$$
\begin{aligned}
\int_{0}^{t} \int_{\Omega \backslash \overline{O(x, \varepsilon)}}\left[v(\xi, \tau) \mathfrak{M}_{\xi, \tau}[u](\xi, \tau)-u(\xi, \tau) \mathfrak{M}_{\xi, \tau}^{t}[v](\xi, \tau)\right] d \xi d \tau= \\
=\int_{0}^{t} \int_{\partial \Omega \cup \partial O(x, \varepsilon)}\left[v(\xi, \tau) \mathfrak{N}_{\xi, \tau}[u](\xi, \tau)-u(\xi, \tau) \mathfrak{N}^{t}[v](\xi, \tau)\right] d S_{\xi} d \tau+ \\
+\int_{\Omega \backslash \overline{O(x, \varepsilon)}}\left[v(\xi, t) \Delta \frac{\partial u}{\partial \tau}(\xi, t)-v(\xi, 0) \Delta \frac{\partial u}{\partial \tau}(\xi, 0)\right] d \xi- \\
\quad-\int_{\Omega \backslash O(x, \varepsilon)}\left[\frac{\partial v}{\partial \tau}(\xi, t) \Delta u(\xi, t)-\frac{\partial v}{\partial \tau}(\xi, 0) \Delta u(\xi, 0)\right] d \xi+ \\
+\int_{\partial \Omega \cup \partial O(x, \varepsilon)}\left[\frac{\partial v}{\partial \tau}(\xi, t) \frac{\partial u}{\partial n_{\xi}}(\xi, t)-\frac{\partial v}{\partial \tau}(\xi, 0) \frac{\partial u}{\partial n_{\xi}}(\xi, 0)\right] d S_{\xi}- \\
-\int_{\partial \Omega \cup \partial(x, \varepsilon)}\left[v(\xi, t) \frac{\partial^{2} u}{\partial \tau \partial n_{\xi}}(\xi, t)-v(\xi, 0) \frac{\partial^{2} u}{\partial \tau \partial n_{\xi}}(\xi, 0)\right] d S_{\xi} .
\end{aligned}
$$

Рассмотрим последовательно все объемные и поверхностные интегралы по $O(x, \varepsilon)$ и по $\partial O(x, \varepsilon)$. Справедлива следующая оценка:

$$
\begin{array}{r}
\left|\int_{0}^{t} \int_{O(x, \varepsilon)} \mathscr{E}(x-\xi, t-\tau) \mathfrak{M}_{\xi, \tau}[u](\xi, \tau) d \xi d \tau\right| \leqslant \sup _{(\xi, \tau) \in \overline{O(x, \varepsilon) \otimes[0, T]}}\left|\mathfrak{M}_{\xi, \tau}[u](\xi, \tau)\right| T \int_{O(x, \varepsilon)} \frac{K_{1}(T)}{|x-\xi|} d \xi \leqslant \\
\leqslant M_{1}(T) T \frac{4 \pi}{3} \varepsilon^{3} \frac{K_{1}(T)}{\varepsilon} \rightarrow+0
\end{array}
$$


при $\varepsilon \rightarrow+0$. Поэтому

$$
\int_{0}^{t} \int_{\Omega \backslash \overline{O(x, \varepsilon)}} \mathscr{E}(x-\xi, t-\tau) \mathfrak{M}_{\xi, \tau}[u](\xi, \tau) d \xi d \tau \rightarrow \int_{0}^{t} \int_{\Omega} \mathscr{E}(x-\xi, t-\tau) \mathfrak{M}_{\xi, \tau}[u](\xi, \tau) d \xi d \tau
$$

при $\varepsilon \rightarrow+0$. В силу (5.19) имеет место равенство

$$
\int_{0}^{t} \int_{\Omega \backslash O(x, \varepsilon)} u(\xi, \tau) \mathfrak{M}_{\xi, \tau}^{t}[v](\xi, \tau) d \xi d \tau=0
$$

Справедлива оценка

$$
\begin{aligned}
\left|\int_{0}^{t} \int_{\partial O(x, \varepsilon)} \mathscr{E}(x-\xi, t-\tau) \mathfrak{N}_{\xi, \tau}[u](\xi, \tau) d \tau d S_{\xi}\right| & \leqslant \\
& \leqslant \sup _{(\xi, \tau) \in \partial O(x, \varepsilon) \otimes[0, T]}\left|\mathfrak{N}_{\xi, \tau}[u](\xi, \tau)\right| \frac{K_{1}(T)}{\varepsilon} 4 \pi \varepsilon^{2} \rightarrow+0
\end{aligned}
$$

при $\varepsilon \rightarrow+0$. Поэтому

$$
\int_{0}^{t} \int_{\partial \Omega \cup \partial O(x, \varepsilon)} \mathscr{E}(x-\xi, t-\tau) \mathfrak{N}_{\xi, \tau}[u](\xi, \tau) d \tau d S_{\xi} \rightarrow \int_{0}^{t} \int_{\partial \Omega} \mathscr{E}(x-\xi, t-\tau) \mathfrak{N}_{\xi, \tau}[u](\xi, \tau) d \tau d S_{\xi}
$$

при $\varepsilon \rightarrow+0$. Имеем следующую цепочку неравенств:

$$
\begin{aligned}
& \left|\int_{0}^{t} \int_{\partial O(x, \varepsilon)} u(\xi, \tau) \mathfrak{N}^{t}[\mathscr{E}(x-\xi, t-\tau)](\xi, \tau) d S_{\xi} d \tau\right| \leqslant \\
& \leqslant \int_{0}^{t} \int_{\partial O(x, \varepsilon)}|u(\xi, \tau)-u(x, \tau)|\left|\mathfrak{N}^{t}[\mathscr{E}(x-\xi, t-\tau)](\xi, \tau)\right| d S_{\xi} d \tau+ \\
& +\left|\int_{0}^{t} u(x, \tau) \int_{\partial O(x, \varepsilon)} \mathfrak{N}^{t}[\mathscr{E}(x-\xi, t-\tau)](\xi, \tau) d S_{\xi} d \tau\right|:=J_{1}+J_{2} .
\end{aligned}
$$

В силу леммы 5.2 имеет место равенство $J_{2}=0$. Для $J_{1}$ справедлива следующая оценка:

$$
J_{1} \leqslant \sup _{(\xi, \tau) \in \partial O(x, \varepsilon) \otimes[0, T]}\left|u_{\xi}^{\prime}(\xi, \tau)\right| \varepsilon T \frac{K_{2}(T)}{\varepsilon^{2}} 4 \pi \varepsilon^{2} \rightarrow+0 \quad \text { при } \varepsilon \rightarrow+0 .
$$

Итак, справедливо предельное свойство

$$
\begin{aligned}
\int_{0}^{t} \int_{\partial \Omega \cup \partial O(x, \varepsilon)} u(\xi, \tau) \mathfrak{N}^{t}[\mathscr{E}(x-\xi, t-\tau)](\xi, \tau) d S_{\xi} d \tau \rightarrow & \\
& \rightarrow \int_{0}^{t} \int_{\partial \Omega} u(\xi, \tau) \mathfrak{N}^{t}[\mathscr{E}(x-\xi, t-\tau)](\xi, \tau) d S_{\xi} d \tau \quad \text { при } \varepsilon \rightarrow+0 .
\end{aligned}
$$

В силу равенств (3.15) теоремы 3.1 имеет место равенство

$$
\int_{O(x, \varepsilon)}\left[\mathscr{E}(x-\xi, 0) \Delta \frac{\partial u}{\partial \tau}(\xi, t)-\mathscr{E}(x-\xi, t) \Delta \frac{\partial u}{\partial \tau}(\xi, 0)\right] d \xi=-\int_{O(x, \varepsilon)} \mathscr{E}(x-\xi, t) \Delta u_{1}(\xi) d \xi,
$$


где $u_{1}(\xi):=\frac{\partial u}{\partial \tau}(\xi, 0)$, причем

$$
\left|\int_{O(x, \varepsilon)} \mathscr{E}(x-\xi, t) \Delta u_{1}(\xi) d \xi\right| \leqslant \sup _{\xi \in O(x, \varepsilon)}\left|\Delta u_{1}(\xi)\right| \frac{4 \pi}{3} \varepsilon^{3} \frac{K_{1}(T)}{\varepsilon} \rightarrow+0 \quad \text { при } \varepsilon \rightarrow+0 .
$$

Поэтому

$$
\int_{\Omega \backslash O(x, \varepsilon)}\left[\mathscr{E}(x-\xi, 0) \Delta \frac{\partial u}{\partial \tau}(\xi, t)-\mathscr{E}(x-\xi, t) \Delta \frac{\partial u}{\partial \tau}(\xi, 0)\right] d \xi \rightarrow-\int_{\Omega} \mathscr{E}(x-\xi, t) \Delta u_{1}(\xi) d \xi
$$

при $\varepsilon \rightarrow+0$. Справедливо следующее соотношение:

$$
\begin{aligned}
-\int_{\Omega \backslash O(x, \varepsilon)}\left[\frac{\partial v}{\partial \tau}(\xi, t) \Delta u(\xi, t)-\frac{\partial v}{\partial \tau}(\xi, 0) \Delta u(\xi, 0)\right] d \xi & = \\
= & \int_{\Omega \backslash O(x, \varepsilon)}\left[\frac{\partial \mathscr{E}}{\partial t}(x-\xi, 0) \Delta u(\xi, t)-\frac{\partial \mathscr{E}}{\partial t}(x-\xi, t) \Delta u_{0}(\xi)\right] d \xi \rightarrow \\
& \rightarrow \int_{\Omega}\left[-\frac{1}{4 \pi|x-\xi|} \Delta u(\xi, t)-\frac{\partial \mathscr{E}}{\partial t}(x-\xi, t) \Delta u_{0}(\xi)\right] d \xi \quad \text { при } \varepsilon \rightarrow+0 ;
\end{aligned}
$$

здесь мы воспользовались теоремой 3.1. Заметим, что в рассматриваемом классе функций $u(x, t)$ справедливо следующее равенство:

$$
u(x, t)=-\int_{\Omega} \frac{\Delta u(\xi, t)}{4 \pi|x-\xi|} d \xi+\int_{\partial \Omega}\left[\frac{1}{4 \pi|x-\xi|} \frac{\partial u(\xi, t)}{\partial n_{\xi}}-u(\xi, t) \frac{\partial}{\partial n_{\xi}} \frac{1}{4 \pi|x-\xi|}\right] d S_{\xi} .
$$

В силу теоремы 3.1 справедливо предельное равенство

$$
\begin{aligned}
\int_{\partial \Omega \cup \partial O(x, \varepsilon)}\left[\frac{\partial v}{\partial \tau}(\xi, t) \frac{\partial u}{\partial n_{\xi}}(\xi, t)-\frac{\partial v}{\partial \tau}(\xi, 0) \frac{\partial u}{\partial n_{\xi}}(\xi, 0)\right] d S_{\xi} \rightarrow \\
\rightarrow \int_{\partial \Omega}\left[-\frac{\partial \mathscr{E}}{\partial t}(x-\xi, 0) \frac{\partial u}{\partial n_{\xi}}(\xi, t)+\frac{\partial \mathscr{E}(x-\xi, t)}{\partial t} \frac{\partial u_{0}(\xi)}{\partial n_{\xi}}\right] d S_{\xi}= \\
=\int_{\partial \Omega}\left[\frac{1}{4 \pi|x-\xi|} \frac{\partial u(\xi, t)}{\partial n_{\xi}}+\frac{\partial \mathscr{E}(x-\xi, t)}{\partial t} \frac{\partial u_{0}(\xi)}{\partial n_{\xi}}\right] d S_{\xi} \quad \text { при } \varepsilon \rightarrow+0 .
\end{aligned}
$$

Наконец, в силу теоремы 3.1 имеет место следующее предельное свойство:

$$
-\int_{\partial \Omega \cup \partial O(x, \varepsilon)}\left[v(\xi, t) \frac{\partial^{2} u}{\partial \tau \partial n_{\xi}}(\xi, t)-v(\xi, 0) \frac{\partial^{2} u}{\partial \tau \partial n_{\xi}}(\xi, 0)\right] d S_{\xi} \rightarrow \int_{\partial \Omega} \mathscr{E}(x-\xi, t) \frac{\partial u_{1}(\xi)}{\partial n_{\xi}} d S_{\xi}
$$

при $\varepsilon \rightarrow+0$. Таким образом, из (5.23), (5.24), (5.26), (5.29), (5.32), (5.33), (5.35) и (5.36) получим следующее равенство:

$$
\begin{aligned}
\int_{0}^{t} \int_{\Omega} \mathscr{E}(x-\xi, t-\tau) \mathfrak{M}_{\xi, \tau}[u](\xi, \tau) d \xi d \tau= \\
\quad=\int_{0}^{t} \int_{\partial \Omega}\left[\mathscr{E}(x-\xi, t-\tau) \mathfrak{N}_{\xi, \tau}[u](\xi, \tau)-u(\xi, \tau) \mathfrak{N}^{t}[\mathscr{E}](x-\xi, t-\tau)\right] d S_{\xi} d \tau-
\end{aligned}
$$




$$
\begin{aligned}
& -\int_{\Omega} \mathscr{E}(x-\xi, t) \Delta u_{1}(\xi) d \xi-\int_{\Omega}\left[\frac{1}{4 \pi|x-\xi|} \Delta u(\xi, t)+\frac{\partial \mathscr{E}(x-\xi, t)}{\partial t} \Delta u_{0}(\xi)\right] d \xi+ \\
& \quad+\int_{\partial \Omega}\left[\frac{1}{4 \pi|x-\xi|} \frac{\partial u(\xi, t)}{\partial n_{\xi}}+\frac{\partial \mathscr{E}(x-\xi, t)}{\partial t} \frac{\partial u_{0}(\xi)}{\partial n_{\xi}}\right] d S_{\xi}+\int_{\partial \Omega} \mathscr{E}(x-\xi, t) \frac{\partial u_{1}(\xi)}{\partial n_{\xi}} d S_{\xi},
\end{aligned}
$$

из которого с учетом равенства (5.34) приходим к соотношению

$$
\begin{aligned}
u(x, t)= & \int_{0}^{t} \int_{\Omega} \mathscr{E}(x-\xi, t-\tau) \mathfrak{M}_{\xi, \tau}[u](\xi, \tau) d \xi d \tau+ \\
& +\int_{0}^{t} \int_{\partial \Omega}\left[u(\xi, \tau) \mathfrak{N}_{\xi, \tau}^{t}[\mathscr{E}](x-\xi, t-\tau)-\mathscr{E}(x-\xi, t-\tau) \mathfrak{N}_{\xi, \tau}[u](\xi, \tau)\right] d S_{\xi} d \tau- \\
- & \int_{\partial \Omega} u(\xi, t) \frac{\partial}{\partial n_{\xi}} \frac{1}{4 \pi|x-\xi|} d S_{\xi}+\int_{\Omega}\left[\mathscr{E}(x-\xi, t) \Delta u_{1}(\xi)+\frac{\partial \mathscr{E}(x-\xi, t)}{\partial t} \Delta u_{0}(\xi)\right] d \xi- \\
& -\int_{\partial \Omega}\left[\mathscr{E}(x-\xi, t) \frac{\partial u_{1}(\xi)}{\partial n_{\xi}}+\frac{\partial \mathscr{E}(x-\xi, t)}{\partial t} \frac{\partial u_{0}(\xi)}{\partial n_{\xi}}\right] d S_{\xi},
\end{aligned}
$$

где операторы $\mathfrak{N}_{\xi, \tau}$ и $\mathfrak{N}_{\xi, \tau}^{t}$ определены равенством (4.5), а оператор $\mathfrak{M}_{x, t}$ равенством (3.1). Таким образом, справедлива следующая теорема.

Теорема 5.1. Для любой функи,ии $u(x, t) \in \mathbb{C}^{(2)}\left([0, T] ; \mathbb{C}^{(2)}(\bar{\Omega})\right), \Omega \subset \mathbb{R}^{3}$-ограниченная область, справедливо равенство (5.38) для $(x, t) \in \Omega \otimes[0, T]$.

\section{6. ТРЕТЬЯ ФОРМУЛА ГРИНА ВО ВСЕМ ПРОСТРАНСТВЕ}

Определение 6.1. Функция $u(x, t) \in \mathbb{C}^{(2)}\left([0, T] ; \mathbb{C}_{b}^{(2)}\left(\mathbb{R}^{3}\right)\right)$ называется регулярной в окрестности бесконечно удаленной точки, если выполнены следующие неравенства:

$$
\begin{gathered}
\left|\frac{\partial^{k} u(x, t)}{\partial t^{k}}\right| \leqslant \frac{M_{1}(T)}{|x|}, \quad\left|\frac{\partial^{k+1} u(x, t)}{\partial t^{k} \partial x_{j}}\right| \leqslant \frac{M_{2}(T)}{|x|^{2}}, \quad k=0,1,2, \quad j=1,2,3, \\
\left|\mathfrak{M}_{x, t}[u](x, t)\right| \leqslant \frac{M_{3}(T)}{|x|^{3}}, \quad \max \left\{\left|\Delta u_{0}(x)\right|,\left|\Delta u_{1}(x)\right|\right\} \leqslant \frac{M_{4}(T)}{|x|^{3}}
\end{gathered}
$$

для всех $t \in[0, T]$ и при $|x| \geqslant R$, где $R>0$ достаточно велико, а постоянные $M_{m}(T)>0$ зависят только от $T>0$ при $m=1,2,3,4$, где использованы обозначения $u_{0}(x):=u(x, 0)$ и $u_{1}(x):=u^{\prime}(x, 0)$.

Справедливо следующее утверждение, которое по своей сути является утверждением о виде третьей формулой Грина во всем пространстве $\mathbb{R}^{3}$ для регулярных функций.

Теорема 6.1. Для любой функиии $и(x, t) \in \mathbb{C}^{(2)}\left([0, T] ; \mathbb{C}_{b}^{(2)}\left(\mathbb{R}^{3}\right)\right)$, регулярной в окрестности бесконечно удаленной точки в смысле определения 6.1 , справедливо равенство

$$
u(x, t)=\int_{0}^{t} \int_{\mathbb{R}^{3}} \mathscr{E}(x-\xi, t-\tau) \mathfrak{M}_{\xi, \tau}[u](\xi, \tau) d \xi d \tau+\int_{\mathbb{R}^{3}}\left[\mathscr{E}(x-\xi, t) \Delta u_{1}(\xi)+\frac{\partial \mathscr{E}(x-\xi, t)}{\partial t} \Delta u_{0}(\xi)\right] d \xi .
$$

Доказательство. Пусть $(x, t) \in \mathbb{R}^{3} \otimes[0, T]$ - произвольная фиксированная точка. Применяя третью формулу Грина $(5.38)$ к ограниченной области $\Omega=O(x, 2 R)$ при $R>0$ и функции $u(x, t) \in \mathbb{C}^{(2)}\left([0, T] ; \mathbb{C}_{b}^{(2)}\left(\mathbb{R}^{3}\right)\right)$, удовлетворяющей условию регулярности в окрестности бесконечно удаленной точки, получим следующую формулу: 


$$
\begin{aligned}
u(x, t)= & \int_{0}^{t} \int_{O(x, 2 R)} \mathscr{E}(x-\xi, t-\tau) \mathfrak{M}_{\xi, \tau}[u](\xi, \tau) d \xi d \tau+ \\
& +\int_{0}^{t} \int_{\partial O(x, 2 R)}\left[u(\xi, \tau) \mathfrak{N}_{\xi, \tau}^{t}[\mathscr{E}](x-\xi, t-\tau)-\mathscr{E}(x-\xi, t-\tau) \mathfrak{N}_{\xi, \tau}[u](\xi, \tau)\right] d S_{\xi} d \tau- \\
- & \int_{\partial O(x, 2 R)} u(\xi, t) \frac{\partial}{\partial n_{\xi}} \frac{1}{4 \pi|x-\xi|} d S_{\xi}+\int_{O(x, 2 R)}\left[\mathscr{E}(x-\xi, t) \Delta u_{1}(\xi)+\frac{\partial \mathscr{E}(x-\xi, t)}{\partial t} \Delta u_{0}(\xi)\right] d \xi- \\
& -\int_{\partial O(x, 2 R)}\left[\mathscr{E}(x-\xi, t) \frac{\partial u_{1}(\xi)}{\partial n_{\xi}}+\frac{\partial \mathscr{E}(x-\xi, t)}{\partial t} \frac{\partial u_{0}(\xi)}{\partial n_{\xi}}\right] d S_{\xi}, \quad(6.4)
\end{aligned}
$$

где $R>0$ таково, что $x \in O(0, R)$. Последовательно рассмотрим все слагаемые в правой части равенства (6.4). Заметим, что в силу (3.13) и (6.2)

$$
\left|\int_{0}^{t} \int_{\mathbb{R}^{3} \backslash O(x, 2 R)} \mathscr{E}(x-\xi, t-\tau) \mathfrak{M}_{\xi, \tau}[u](\xi, \tau) d \xi d \tau\right| \leqslant T \int_{\mathbb{R}^{3} \backslash O(x, 2 R)} \frac{K_{1}(T)}{|x-\xi|} \frac{M_{3}(T)}{|\xi|^{3}} d \xi:=I_{1}(R),
$$

поскольку при $\xi \in \mathbb{R}^{3} \backslash O(x, 2 R)$ и $x \in O(0, R)$ имеем $|\xi| \geqslant|x-\xi|-|x| \geqslant 2 R-R=R$. После замены $\xi_{j}-x_{j}=y_{j}$ получим следующее выражение для $I_{1}(R)$ :

$$
I_{1}(R):=T K_{1}(T) M_{3}(T) \int_{\mathbb{R}^{3} \backslash O(0,2 R)} \frac{1}{|x+y|^{3}|y|} d y .
$$

Для оценки этого интеграла заметим, что в силу нашего выбора справедливы следующие неравенства:

$$
|x| \leqslant R \leqslant \frac{|y|}{2} \Rightarrow|x+y| \geqslant|y|-|x| \geqslant \frac{|y|}{2} .
$$

Поэтому справедлива следующая оценка:

$$
I_{1}(R) \leqslant K_{4}(T) \int_{\mathbb{R}^{3} \backslash O(0,2 R)} \frac{1}{|y|^{4}} d y=K_{4}(T) 4 \pi \int_{2 R}^{+\infty} \frac{1}{r^{2}} d r \rightarrow+0 \quad \text { при } R \rightarrow+\infty .
$$

Таким образом,

$$
\int_{0}^{t} \int_{O(x, 2 R)} \mathscr{E}(x-\xi, t-\tau) \mathfrak{M}_{\xi, \tau}[u](\xi, \tau) d \xi d \tau \rightarrow \int_{0}^{t} \int_{\mathbb{R}^{3}} \mathscr{E}(x-\xi, t-\tau) \mathfrak{M}_{\xi, \tau}[u](\xi, \tau) d \xi d \tau
$$

при $R \rightarrow+\infty$. Справедлива следующая оценка:

$$
\begin{aligned}
\left|\int_{0}^{t} \int_{\partial O(x, 2 R)} u(\xi, \tau) \mathfrak{N}_{\xi, \tau}^{t}[\mathscr{E}](x-\xi, t-\tau) d S_{\xi} d \tau\right| \leqslant T & \int_{|x-\xi|=2 R} \frac{M_{1}(T)}{|\xi|} \max \left\{1, \omega_{1}^{2}, \omega_{2}^{2}, \omega_{3}^{2}\right\} \frac{4 K_{2}(T)}{|x-\xi|^{2}} d \xi \leqslant \\
& \leqslant \frac{K_{5}(T)}{R^{3}} 4 \pi R^{2} \rightarrow+0 \quad \text { при } R \rightarrow+\infty, \quad \text { (6.10) }
\end{aligned}
$$

где мы воспользовались неравенствами $|\xi| \geqslant|x-\xi|-|x| \geqslant 2 R-R=R$. Аналогичным образом доказывается, что

$$
\int_{0}^{t} \int_{\partial O(x, 2 R)} \mathscr{E}(x-\xi, t-\tau) \mathfrak{N}_{\xi, \tau}[u](\xi, \tau) d S_{\xi} d \tau \rightarrow 0 \quad \text { при } R \rightarrow+\infty .
$$


Поскольку справедлива оценка

$$
\left|\frac{\partial}{\partial n_{\xi}} \frac{1}{|x-\xi|}\right| \leqslant \frac{c_{1}}{|x-\xi|^{2}}, \quad \xi \neq x
$$

то имеет место предельное свойство

$$
\int_{\partial O(x, 2 R)} u(\xi, t) \frac{\partial}{\partial n_{\xi}} \frac{1}{4 \pi|x-\xi|} d S_{\xi} \rightarrow 0 \quad \text { при } R \rightarrow+\infty .
$$

Точно так же, как при выводе предельного свойства (6.9), с учетом свойств (3.13) и (6.2) можно доказать, что

$$
\begin{aligned}
& \int_{O(x, 2 R)}\left[\mathscr{E}(x-\xi, t) \Delta u_{1}(\xi)\right.\left.+\frac{\partial \mathscr{E}(x-\xi, t)}{\partial t} \Delta u_{0}(\xi)\right] d \xi \rightarrow \\
& \rightarrow \int_{\mathbb{R}^{3}}\left[\mathscr{E}(x-\xi, t) \Delta u_{1}(\xi)+\frac{\partial \mathscr{E}(x-\xi, t)}{\partial t} \Delta u_{0}(\xi)\right] d \xi \quad \text { при } R \rightarrow+\infty .
\end{aligned}
$$

Наконец, нетрудно доказать, что

$$
\int_{\partial O(x, 2 R)}\left[\mathscr{E}(x-\xi, t) \frac{\partial u_{1}(\xi)}{\partial n_{\xi}}+\frac{\partial \mathscr{E}(x-\xi, t)}{\partial t} \frac{\partial u_{0}(\xi)}{\partial n_{\xi}}\right] d S_{\xi} \rightarrow 0 \quad \text { при } R \rightarrow+\infty .
$$

Таким образом, при $R \rightarrow+\infty$ из (6.4) получим равенство (6.3). Теорема 6.1 доказана.

\section{7. СЛАБЫЕ РЕШЕНИЯ ЗАДАЧ КОШИ}

В этом разделе мы приведем дадим слабые формулировки следующих задач Коши:

$$
\begin{gathered}
\frac{\partial^{2}}{\partial t^{2}} \Delta u+\omega_{1}^{2} u_{x_{1} x_{1}}+\omega_{2}^{2} u_{x_{2} x_{2}}+\omega_{3}^{2} u_{x_{3} x_{3}}+|\nabla u|^{q}=0, \quad(x, t) \in \mathbb{R}^{3} \otimes(0, T], \\
u(x, 0)=u_{0}(x), \quad u^{\prime}(x, 0)=u_{1}(x), \quad x \in \mathbb{R}^{3} ; \\
\frac{\partial^{2}}{\partial t^{2}} \Delta u+\omega_{1}^{2} u_{x_{1} x_{1}}+\omega_{2}^{2} u_{x_{2} x_{2}}+\omega_{3}^{2} u_{x_{3} x_{3}}-\frac{\partial|\nabla u|^{q}}{\partial t}=0, \quad(x, t) \in \mathbb{R}^{3} \otimes(0, T], \\
u(x, 0)=u_{0}(x), \quad u^{\prime}(x, 0)=u_{1}(x), \quad x \in \mathbb{R}^{3} ; \\
\frac{\partial^{2}}{\partial t^{2}} \Delta u+\omega_{1}^{2} u_{x_{1} x_{1}}+\omega_{2}^{2} u_{x_{2} x_{2}}+\omega_{3}^{2} u_{x_{3} x_{3}}+\frac{\partial^{2}|\nabla u|^{q}}{\partial t^{2}}=0, \quad(x, t) \in \mathbb{R}^{3} \otimes(0, T], \\
u(x, 0)=u_{0}(x), \quad u^{\prime}(x, 0)=u_{1}(x), \quad x \in \mathbb{R}^{3} .
\end{gathered}
$$

Определение 7.1. Слабым локальным во времени решением задачи Коши (7.1), (7.2) называется такая функция $u(x, t) \in W_{q, \mathrm{loc}}^{1,0}\left(\mathbb{R}^{3} \otimes[0, T]\right)$, что для любой функции $\phi(x, t) \in \mathscr{D}\left(\mathbb{R}^{3} \otimes(-\infty, T)\right)$ справедливо следующее равенство:

$$
\begin{aligned}
& \int_{0}^{T} \int_{\mathbb{R}^{3}}\left[\left(\nabla u(x, t), \nabla \frac{\partial^{2} \phi(x, t)}{\partial t^{2}}\right)+\sum_{j=1}^{3} \omega_{j}^{2} u_{x_{j}}(x, t) \phi_{x_{j}}(x, t)\right] d x d t+ \\
& \quad+\int_{\mathbb{R}^{3}}\left[\left(\nabla \frac{\partial \phi}{\partial t}(x, 0), \nabla u_{0}(x)\right)-\left(\nabla \phi(x, 0), \nabla u_{1}(x)\right)\right] d x=\int_{0}^{T} \int_{\mathbb{R}^{3}} \phi(x, t)|\nabla u(x, t)|^{q} d x d t,
\end{aligned}
$$

причем $u_{0}(x), u_{1}(x) \in W_{1, \mathrm{loc}}^{1,0}\left(\mathbb{R}^{3}\right)$. 
Определение 7.2. Слабым глобальным во времени решением задачи Коши (7.1), (7.2) называется такая функция $u(x, t) \in W_{q, \mathrm{loc}}^{1,0}\left(\mathbb{R}^{3} \otimes[0,+\infty)\right)$, что для любой функции $\phi(x, t) \in$ $\mathscr{D}\left(\mathbb{R}^{3} \otimes(-\infty,+\infty)\right)$ справедливо следующее равенство:

$$
\begin{aligned}
& \int_{0}^{+\infty} \int_{\mathbb{R}^{3}} {\left[\left(\nabla u(x, t), \nabla \frac{\partial^{2} \phi(x, t)}{\partial t^{2}}\right)+\sum_{j=1}^{3} \omega_{j}^{2} u_{x_{j}}(x, t) \phi_{x_{j}}(x, t)\right] d x d t+} \\
&+\int_{\mathbb{R}^{3}}\left[\left(\nabla \frac{\partial \phi}{\partial t}(x, 0), \nabla u_{0}(x)\right)-\left(\nabla \phi(x, 0), \nabla u_{1}(x)\right)\right] d x=\int_{0}^{+\infty} \int_{\mathbb{R}^{3}} \phi(x, t)|\nabla u(x, t)|^{q} d x d t,
\end{aligned}
$$

причем $u_{0}(x), u_{1}(x) \in W_{1, \mathrm{loc}}^{1,0}\left(\mathbb{R}^{3}\right)$.

Определение 7.3. Слабым локальным во времени решением задачи Коши (7.3), (7.4) называется такая функция $u(x, t) \in W_{q, \mathrm{loc}}^{1,0}\left(\mathbb{R}^{3} \otimes[0, T]\right)$, что для любой функции $\phi(x, t) \in \mathscr{D}\left(\mathbb{R}^{3} \otimes(-\infty, T)\right)$ справедливо следующее равенство:

$$
\begin{gathered}
\int_{0}^{T} \int_{\mathbb{R}^{3}}\left[\left(\nabla u(x, t), \nabla \frac{\partial^{2} \phi(x, t)}{\partial t^{2}}\right)+\sum_{j=1}^{3} \omega_{j}^{2} u_{x_{j}}(x, t) \phi_{x_{j}}(x, t)\right] d x d t+\int_{\mathbb{R}^{3}}\left[\left(\nabla \frac{\partial \phi}{\partial t}(x, 0), \nabla u_{0}(x)\right)-\right. \\
\left.-\left(\nabla \phi(x, 0), \nabla u_{1}(x)\right)-\phi(x, 0)\left|\nabla u_{0}(x)\right|^{q}\right] d x=\int_{0}^{T} \int_{\mathbb{R}^{3}} \frac{\partial \phi(x, t)}{\partial t}|\nabla u(x, t)|^{q} d x d t
\end{gathered}
$$

причем $u_{0}(x) \in W_{q, \mathrm{loc}}^{1,0}\left(\mathbb{R}^{3}\right), u_{1}(x) \in W_{1, \mathrm{loc}}^{1,0}\left(\mathbb{R}^{3}\right)$.

Определение 7.4. Слабым глобальным во времени решением задачи Коши (7.3), (7.4) называется такая функция $u(x, t) \in W_{q, \text { loc }}^{1,0}\left(\mathbb{R}^{3} \otimes[0,+\infty)\right)$, что для любой функции $\phi(x, t) \in$ $\mathscr{D}\left(\mathbb{R}^{3} \otimes(-\infty,+\infty)\right)$ справедливо следующее равенство:

$$
\begin{array}{r}
\int_{0}^{+\infty} \int_{\mathbb{R}^{3}}\left[\left(\nabla u(x, t), \nabla \frac{\partial^{2} \phi(x, t)}{\partial t^{2}}\right)+\sum_{j=1}^{3} \omega_{j}^{2} u_{x_{j}}(x, t) \phi_{x_{j}}(x, t)\right] d x d t+\int_{\mathbb{R}^{3}}\left[\left(\nabla \frac{\partial \phi}{\partial t}(x, 0), \nabla u_{0}(x)\right)-\right. \\
\left.-\left(\nabla \phi(x, 0), \nabla u_{1}(x)\right)-\phi(x, 0)\left|\nabla u_{0}(x)\right|^{q}\right] d x=\int_{0}^{+\infty} \int_{\mathbb{R}^{3}} \frac{\partial \phi(x, t)}{\partial t}|\nabla u(x, t)|^{q} d x d t, \quad(7.10
\end{array}
$$

причем $u_{0}(x) \in W_{q, \mathrm{loc}}^{1,0}\left(\mathbb{R}^{3}\right), u_{1}(x) \in W_{1, \mathrm{loc}}^{1,0}\left(\mathbb{R}^{3}\right)$.

Определение 7.5. Слабым локальным во времени решением задачи Коши (7.5), (7.6) называется такая функция $u(x, t) \in W_{q, \text { loc }}^{1,0}\left(\mathbb{R}^{3} \otimes[0, T]\right)$, что для любой функции $\phi(x, t) \in \mathscr{D}\left(\mathbb{R}^{3} \otimes(-\infty, T)\right)$ справедливо следующее равенство:

$$
\begin{array}{r}
\int_{0}^{T} \int_{\mathbb{R}^{3}}\left[\left(\nabla u(x, t), \nabla \frac{\partial^{2} \phi(x, t)}{\partial t^{2}}\right)+\sum_{j=1}^{3} \omega_{j}^{2} u_{x_{j}}(x, t) \phi_{x_{j}}(x, t)\right] d x d t+\int_{\mathbb{R}^{3}}\left[\left(\nabla \frac{\partial \phi}{\partial t}(x, 0), \nabla u_{0}(x)\right)-\right. \\
-\left(\nabla \phi(x, 0), \nabla u_{1}(x)\right)-\frac{\partial \phi}{\partial t}(x, 0)\left|\nabla u_{0}(x)\right|^{q}+\phi(x, 0) q \\
\left.\left(\nabla u_{1}(x),\left|\nabla u_{0}(x)\right|^{q-2} \nabla u_{0}(x)\right)\right] d x= \\
=\int_{0}^{T} \int_{\mathbb{R}^{3}} \frac{\partial^{2} \phi(x, t)}{\partial t^{2}}|\nabla u(x, t)|^{q} d x d t,
\end{array}
$$

причем $u_{0}(x), u_{1}(x) \in W_{q, \mathrm{loc}}^{1,0}\left(\mathbb{R}^{3}\right)$. 
Определение 7.6. Слабым глобальным во времени решением задачи Коши (7.5), (7.6) называется такая функция $u(x, t) \in W_{q, \text { loc }}^{1,0}\left(\mathbb{R}^{3} \otimes[0,+\infty)\right)$, что для любой функции $\phi(x, t) \in$ $\mathscr{D}\left(\mathbb{R}^{3} \otimes(-\infty,+\infty)\right)$ справедливо следующее равенство:

$$
\begin{aligned}
& \int_{0}^{+\infty} \int_{\mathbb{R}^{3}}\left[\left(\nabla u(x, t), \nabla \frac{\partial^{2} \phi(x, t)}{\partial t^{2}}\right)+\sum_{j=1}^{3} \omega_{j}^{2} u_{x_{j}}(x, t) \phi_{x_{j}}(x, t)\right] d x d t+ \\
&+\int_{\mathbb{R}^{3}}\left[\left(\nabla \frac{\partial \phi}{\partial t}(x, 0), \nabla u_{0}(x)\right)-\left(\nabla \phi(x, 0), \nabla u_{1}(x)\right)-\right. \\
&\left.-\frac{\partial \phi}{\partial t}(x, 0)\left|\nabla u_{0}(x)\right|^{q}+\phi(x, 0) q\left(\nabla u_{1}(x),\left|\nabla u_{0}(x)\right|^{q-2} \nabla u_{0}(x)\right)\right] d x= \\
& \quad=\int_{0}^{+\infty} \int_{\mathbb{R}^{3}} \frac{\partial^{2} \phi(x, t)}{\partial t^{2}}|\nabla u(x, t)|^{q} d x d t,
\end{aligned}
$$

причем $u_{0}(x), u_{1}(x) \in W_{q, \text { loc }}^{1,0}\left(\mathbb{R}^{3}\right)$.

Заметим, что имеется связь между введенными локальными во времени слабыми решениями в смысле определений $7.1,7.3,7.5$ и глобальными во времени слабыми решениями в смысле определений $7.2,7.4,7.6$, соответственно.

Лемма 7.1. Всякие глобальные во времени слабые решения в смысле определений 7.2, 7.4, 7.6 являются локальными во времени слабыми решениями в смысле определений 7.1, 7.3, 7.5 соответственно.

Доказательство основано на том, что

$$
W_{q, \text { loc }}^{1,0}\left(\mathbb{R}^{3} \otimes[0,+\infty)\right) \subset W_{q, \text { loc }}^{1,0}\left(\mathbb{R}^{3} \otimes[0, T]\right)
$$

для любого $T>0$ и, кроме того,

$$
\mathscr{D}\left(\mathbb{R}^{3} \otimes(-\infty, T)\right) \subset \mathscr{D}\left(\mathbb{R}^{3} \otimes(-\infty,+\infty)\right),
$$

если функции из $\mathscr{D}\left(\mathbb{R}^{3} \otimes(-\infty, T)\right)$ продолжить нулем при $t \geqslant T$.

Теорема 7.1. Пусть и $(x, t)$ - слабое решение задачи Коши (7.1), (7.2) в смысле определения 7.1. Тогда в классе таких функиий $u(x, t), u_{0}(x)$ и $u_{1}(x)$, что существуют свертки

$$
\begin{aligned}
U_{1}(x, t) & :=-\int_{-\infty} \int_{\mathbb{R}^{3}} \mathscr{E}(x-y, t-\tau)|\nabla \widetilde{u}(y, \tau)|^{q} d y d \tau \in L_{\mathrm{loc}}^{1}\left(\mathbb{R}^{3} \otimes(-\infty, T)\right), \\
V(x, t) & :=\int_{\mathbb{R}^{3}} \mathscr{E}(x-y, t) \Delta u_{1}(y) d y \in L_{\mathrm{loc}}^{1}\left(\mathbb{R}^{3} \otimes(-\infty, T)\right), \\
W(x, t) & :=\int_{\mathbb{R}^{3}} \frac{\partial \mathscr{E}(x-y, t)}{\partial t} \Delta u_{0}(y) d y \in L_{\mathrm{loc}}^{1}\left(\mathbb{R}^{3} \otimes(-\infty, T)\right),
\end{aligned}
$$

справедливо следующее представление в виде суммы трех потени,илов:

$$
\widetilde{u}(x, t)=U_{1}(x, t)+V(x, t)+W(x, t) \quad \text { для п.в. }(x, t) \in \mathbb{R}^{3} \otimes(-\infty, T),
$$

где $\mathscr{E}(x, t)$ - фундаментальное решение оператора $\mathfrak{M}_{x, t}$, определенное равенством (3.12). 
Доказательство. Пусть $u(x, t)$ - локальное во времени слабое решение задачи Коши (7.1), (7.2), а $\phi(x, t) \in \mathscr{D}\left(\mathbb{R}^{3} \otimes(-\infty, T)\right)$ - произвольная функция. Рассмотрим продолжение этой функции

$$
\widetilde{u}(x, t)= \begin{cases}u(x, t), & \text { если } t \geqslant 0, \\ 0, & \text { если } t<0 .\end{cases}
$$

Отметим, что тогда

$$
\widetilde{|\nabla u|^{q}}(x, t)=\left\{\begin{array}{ll}
|\nabla u(x, t)|^{q}, & \text { если } t \geqslant 0, \\
0, & \text { если } t<0 .
\end{array}=|\nabla \widetilde{u}|^{q} .\right.
$$

Справедлива следующая цепочка равенств:

$$
\begin{gathered}
\left\langle\mathfrak{M}_{x, t}[\widetilde{u}](x, t), \phi(x, t)\right\rangle=\left\langle\widetilde{u}(x, t), \mathfrak{M}_{x, t}[\phi(x, t)]\right\rangle=\int_{0}^{T} \int_{\mathbb{R}^{3}} u(x, t) \mathfrak{M}_{x, t}[\phi](x, t) d x d t= \\
=-\int_{0} \int_{\mathbb{R}^{3}}\left[\left(\nabla u(x, t), \nabla \frac{\partial^{2} \phi(x, t)}{\partial t^{2}}\right)+\sum_{j=1}^{3} \omega_{j}^{2} u_{x_{j}}(x, t) \phi_{x_{j}}(x, t)\right] d x d t= \\
=\int_{\mathbb{R}^{3}}\left[\left(\nabla \frac{\partial \phi}{\partial t}(x, 0), \nabla u_{0}(x)\right)-\left(\nabla \phi(x, 0), \nabla u_{1}(x)\right)\right] d x-\int_{0}^{T} \int_{\mathbb{R}^{3}} \phi(x, t)|\nabla u(x, t)|^{q} d x d t= \\
=\int_{\mathbb{R}^{3}} u_{1}(x) \Delta \phi(x, 0) d x-\int_{\mathbb{R}^{3}} u_{0}(x) \Delta \frac{\partial \phi}{\partial t}(x, 0) d x-\int_{-\infty}^{T} \int_{\mathbb{R}^{3}} \phi(x, t)|\nabla \widetilde{u}(x, t)|^{q} d x d t= \\
=\left\langle u_{1}(x) \delta(t), \Delta \phi(x, t)\right\rangle+\left\langle u_{0}(x) \delta^{\prime}(t), \Delta \phi(x, t)\right\rangle-\left\langle|\nabla \widetilde{u}(x, t)|^{q}, \phi(x, t)\right\rangle= \\
=\left\langle\delta(t) \Delta u_{1}(x)+\delta^{\prime}(t) \Delta u_{0}(x)-|\nabla \widetilde{u}(x, t)|^{q}, \phi(x, t)\right\rangle .
\end{gathered}
$$

Итак, справедливо равенство

$$
\left\langle\mathfrak{M}_{x, t}[\widetilde{u}](x, t), \phi(x, t)\right\rangle=\left\langle\delta(t) \Delta u_{1}(x)+\delta^{\prime}(t) \Delta u_{0}(x)-|\nabla \widetilde{u}(x, t)|^{q}, \phi(x, t)\right\rangle,
$$

в котором все производные понимаются в смысле обобщенных функций из $\mathscr{D}^{\prime}\left(\mathbb{R}^{3} \otimes(-\infty, T)\right)$. Таким образом, мы приходим к следующему равенству, понимаемому в смысле обобщенных функций:

$$
\mathfrak{M}_{x, t}[\widetilde{u}](x, t)=\delta(t) \Delta u_{1}(x)+\delta^{\prime}(t) \Delta u_{0}(x)-|\nabla \widetilde{u}(x, t)|^{q} .
$$

В силу [1, теорема 11.3$]$ приходим к утверждению теоремы.

Теорема 7.2. Пусть и $(x, t)$ - слабое решение задачи Коши (7.3), (7.4) в смысле определения 7.3. Тогда в классе $|\nabla u(x, t)|^{q} \in \mathbb{C}_{x, t}^{(0,1)}\left(\mathbb{R}^{3} \otimes[0, T]\right)$ таких функиий $u(x, t), u_{0}(x)$ и $u_{1}(x)$, что существуют свертки

$$
\begin{aligned}
U_{2}(x, t) & :=\int_{-\infty}^{t} \int_{\mathbb{R}^{3}} \mathscr{E}(x-y, t-\tau)\left\{\frac{\partial|\nabla \widetilde{u}(y, \tau)|^{q}}{\partial \tau}\right\} d y d \tau \in L_{\mathrm{loc}}^{1}\left(\mathbb{R}^{3} \otimes(-\infty, T)\right), \\
V(x, t) & :=\int_{\mathbb{R}^{3}} \mathscr{E}(x-y, t) \Delta u_{1}(y) d y \in L_{\mathrm{loc}}^{1}\left(\mathbb{R}^{3} \otimes(-\infty, T)\right), \\
W(x, t) & :=\int_{\mathbb{R}^{3}} \frac{\partial \mathscr{E}(x-y, t)}{\partial t} \Delta u_{0}(y) d y \in L_{\mathrm{loc}}^{1}\left(\mathbb{R}^{3} \otimes(-\infty, T)\right),
\end{aligned}
$$


справедливо следующее представление в виде суммы трех потенциалов:

$$
\widetilde{u}(x, t)=U_{2}(x, t)+V(x, t)+W(x, t) \quad \text { для n.в. }(x, t) \in \mathbb{R}^{3} \otimes(-\infty, T),
$$

где $\mathscr{E}(x, t)$ - фундаментальное решение оператора $\mathfrak{M}_{x, t}$, определенное равенством (3.12).

Доказательство. Справедлива следующая цепочка равенств:

$$
\begin{aligned}
& \left\langle\mathfrak{M}_{x, t}[\widetilde{u}](x, t), \phi(x, t)\right\rangle=\left\langle\widetilde{u}, \mathfrak{M}_{x, t}[\phi](x, t)\right\rangle= \\
& =-\int_{0} \int_{\mathbb{R}^{3}}\left[\left(\nabla u(x, t), \nabla \frac{\partial^{2} \phi(x, t)}{\partial t^{2}}\right)+\sum_{j=1}^{3} \omega_{j}^{2} u_{x_{j}}(x, t) \phi_{x_{j}}(x, t)\right] d x d t= \\
& =-\int_{0}^{T} \int_{\mathbb{R}^{3}} \frac{\partial \phi(x, t)}{\partial t}|\nabla u(x, t)|^{q} d x d t+\int_{\mathbb{R}^{3}}\left[\left(\nabla \frac{\partial \phi}{\partial t}(x, 0), \nabla u_{0}(x)\right)-\right. \\
& =-\int_{-\infty}^{T} \frac{\partial \phi(x, t)}{\partial t}|\nabla \widetilde{u}(x, t)|^{q} d x d t+\int_{\mathbb{R}^{3}}\left[\left(\nabla \frac{\partial \phi}{\partial t}(x, 0), \nabla u_{0}(x)\right)-\right. \\
& \left.=-\left\langle|\nabla \widetilde{u}(x, t)|^{q}, \frac{\partial \phi(x, t)}{\partial t}\right\rangle+\left\langle\Delta u_{1}(x)\right)-\phi(x, 0)\left|\nabla u_{0}(x)\right|^{q}\right] d x= \\
& =\left\langle\frac{d}{d t}|\nabla \widetilde{u}(x, t)|^{q}, \phi(x, t)\right\rangle-\left\langle\left|\nabla u_{0}(x)\right|^{q} \delta(t), \phi(x, t)\right\rangle+\left\langle\Delta u_{0}(x) \delta^{\prime}(t)+\Delta u_{1}(x) \delta^{\prime}(t), \phi(x, t)\right\rangle .
\end{aligned}
$$

Предположим, что $|\nabla u(x, t)|^{q} \in \mathbb{C}_{x, t}^{(0,1)}\left(\mathbb{R}^{3} \otimes[0, T]\right)$. Тогда справедлива следующая цепочка равенств:

$$
\begin{gathered}
\left\langle\frac{d}{d t}|\nabla \widetilde{u}(x, t)|^{q}, \phi(x, t)\right\rangle=-\left\langle|\nabla \widetilde{u}(x, t)|^{q}, \frac{\partial \phi(x, t)}{\partial t}\right\rangle=-\int_{0}^{T} \int_{\mathbb{R}^{3}}|\nabla u(x, t)|^{q} \frac{\partial \phi(x, t)}{\partial t} d x d t= \\
=\int_{\mathbb{R}^{3}}\left|\nabla u_{0}(x)\right|^{q} \phi(x, 0) d x+\int_{0}^{T} \int_{\mathbb{R}^{3}}^{T}\left\{\frac{\partial}{\partial t}|\nabla u(x, t)|^{q}\right\} \phi(x, t) d x d t= \\
=\int_{\mathbb{R}^{3}}\left|\nabla u_{0}(x)\right|^{q} \phi(x, 0) d x+\int_{-\infty}^{T} \int_{\mathbb{R}^{3}}\left\{\frac{\partial}{\partial t}|\nabla \widetilde{u}(x, t)|^{q}\right\} \phi(x, t) d x d t= \\
=\left\langle\left|\nabla u_{0}(x)\right|^{q} \delta(t), \phi(x, t)\right\rangle+\left\langle\left\{\frac{\partial}{\partial t}|\nabla \widetilde{u}(x, t)|^{q}\right\}, \phi(x, t)\right\rangle .
\end{gathered}
$$

Тогда из равенств (7.26) и (7.27) вытекает следующее равенство:

$$
\left\langle\mathfrak{M}_{x, t}[\widetilde{u}](x, t), \phi(x, t)\right\rangle=\left\langle\left\{\frac{\partial}{\partial t}|\nabla \widetilde{u}(x, t)|^{q}\right\}+\delta^{\prime}(t) \Delta u_{0}(x)+\delta(t) \Delta u_{1}(x), \phi(x, t)\right\rangle
$$

для любых функций $\phi(x, t) \in \mathscr{D}\left(\mathbb{R}^{3} \otimes(-\infty, T)\right)$. Таким образом, всякое слабое решение задачи Коши (7.3), (7.4) в смысле определения 7.3 удовлетворяет следующему равенству в смысле 
пространства обобщенных функций $\mathscr{D}^{\prime}\left(\mathbb{R}^{3} \otimes(-\infty, T)\right)$ :

$$
\mathfrak{M}_{x, t}[\widetilde{u}](x, t)=\left\{\frac{\partial}{\partial t}|\nabla \widetilde{u}(x, t)|^{q}\right\}+\delta^{\prime}(t) \Delta u_{0}(x)+\delta(t) \Delta u_{1}(x),
$$

где все производные понимаются в смысле обобщенных функций из $\mathscr{D}^{\prime}\left(\mathbb{R}^{3} \otimes(-\infty, T)\right)$. Применяя теорему 11.3 из [1], приходим к утверждению теоремы.

Аналогичным образом можно доказать следующее утверждение.

Теорема 7.3. Пусть $и(x, t)$ - слабое решение задачи Коши (7.5), (7.6) в смысле определения 7.5. Тогда в классе $|\nabla u(x, t)|^{q} \in \mathbb{C}_{x, t}^{(0,2)}\left(\mathbb{R}^{3} \otimes[0, T]\right)$ таких функиий $u(x, t), u_{0}(x)$ и $u_{1}(x)$, что существуют свертки

$$
\begin{aligned}
U_{3}(x, t) & :=-\int_{-\infty}^{t} \int_{\mathbb{R}^{3}} \mathscr{E}(x-y, t-\tau)\left\{\frac{\partial^{2}|\nabla \widetilde{u}(y, \tau)|^{q}}{\partial \tau^{2}}\right\} d y d \tau \in L_{\mathrm{loc}}^{1}\left(\mathbb{R}^{3} \otimes(-\infty, T)\right), \\
V(x, t) & :=\int_{\mathbb{R}^{3}} \mathscr{E}(x-y, t) \Delta u_{1}(y) d y \in L_{\mathrm{loc}}^{1}\left(\mathbb{R}^{3} \otimes(-\infty, T)\right), \\
W(x, t) & :=\int_{\mathbb{R}^{3}} \frac{\partial \mathscr{E}(x-y, t)}{\partial t} \Delta u_{0}(y) d y \in L_{\mathrm{loc}}^{1}\left(\mathbb{R}^{3} \otimes(-\infty, T)\right),
\end{aligned}
$$

справедливо следующее представление в виде суммы трех потени,иалов:

$$
\widetilde{u}(x, t)=U_{3}(x, t)+V(x, t)+W(x, t) \quad \text { для n.в. }(x, t) \in \mathbb{R}^{3} \otimes(-\infty, T),
$$

где $\mathscr{E}(x, t)$ - фундаментальное решение оператора $\mathfrak{M}_{x, t}$, определенное равенством (3.12).

\section{8. СВОЙСТВА ОБЪЕМНОГО И ПОВЕРХНОСТНЫХ ПОТЕНЦИАЛОВ}

Рассмотрим следующие объемный и поверхностные потенциалы, имеющие важную роль при исследовании локальной во времени разрешимости поставленных в предыдущем разделе задач Коши:

$$
\begin{aligned}
U(x, t) & :=U[\rho](x, t):=\int_{0} \int_{\mathbb{R}^{3}} G_{\beta}(x, y, t-\tau) \rho(y, \tau) d y d \tau, \\
V(x, t) & :=V[\mu](x, t):=\int_{\mathbb{R}^{3}} G_{\beta}(x, y, t) \mu(y) d y \\
W(x, t) & :=W[\sigma](x, t):=\int_{\mathbb{R}^{3}} \frac{\partial G_{\beta}(x, y, t)}{\partial t} \sigma(y) d y,
\end{aligned}
$$

где

$$
G_{\beta}(x, y, t):=\frac{\left(1+|x|^{2}\right)^{1 / 2}}{\left(1+|y|^{2}\right)^{\beta}} \mathscr{E}(x-y, t), \quad \beta>\frac{3}{2},
$$

а $\mathscr{E}(x, t)$ - фундаментальное решение, определенное равенством (3.12). Сначала рассмотрим объемный потенциал $U(x, t)$, определенный равенством (8.1). Отметим, что его можно записать в виде

$$
U(x, t)=\int_{0}^{t} H(x, t, \tau) d \tau, \quad H(x, t, \tau):=\int_{\mathbb{R}^{3}} G_{\beta}(x, y, t-\tau) \rho(y, \tau) d y .
$$

Справедлива следующая теорема.

Теорема 8.1. Если $\rho(x, t) \in \mathbb{C}\left([0, T] ; \mathbb{C}_{b}\left(\mathbb{R}^{3}\right)\right)$, то при условии $\beta>3 / 2$ оббемный потенииал $U(x, t)$ принадлежит классу $\mathbb{C}^{(2)}\left([0, T] ; \mathbb{C}_{b}^{1,0}\left(\left(1+|x|^{2}\right)^{1 / 2} ; \mathbb{R}^{3}\right)\right)$. 
Доказательство. Шаг 1. Поскольку $\rho(x, t) \in \mathbb{C}\left([0, T] ; \mathbb{C}_{b}\left(\mathbb{R}^{3}\right)\right)$, то справедливо неравенство

$$
\sup _{(x, t) \in \mathbb{R}^{3} \otimes[0, T]}|\rho(x, t)|<+\infty .
$$

Докажем, что $H(x, t, \tau) \in \mathbb{C}_{b}\left(\mathbb{R}^{3} \otimes[0, t]\right)$ для любого $t \in(0, T]$. Действительно, пусть $\left(x^{1}, \tau^{1}\right),\left(x^{2}, \tau^{2}\right) \in \mathbb{R}^{3} \otimes[0, t]$. Заметим, что для любых $\varepsilon_{0}>0$ и $R_{0}>\varepsilon_{0}$ справедливо равенство

$$
H\left(x^{j}, t, \tau^{j}\right)=H_{1}\left(x^{j}, t, \tau^{j}\right)+H_{2}\left(x^{j}, t, \tau^{j}\right)+H_{3}\left(x^{j}, t, \tau^{j}\right)
$$

где

$$
\begin{aligned}
& H_{1}\left(x^{j}, t, \tau^{j}\right)=\int_{\mathbb{R}^{3} \backslash O\left(x^{j}, R_{0}\right)} G_{\beta}\left(x^{j}, y, t-\tau^{j}\right) \rho\left(y, \tau^{j}\right) d y, \\
& H_{2}\left(x^{j}, t, \tau^{j}\right)= \\
& \int_{3}\left(x^{j}, t, \tau^{j}\right)=\int_{O\left(x^{j}, R_{0}\right) \backslash O\left(x^{j}, \varepsilon_{0}\right)} G_{\beta}\left(x^{j}, y, t-\tau^{j}\right) \rho\left(y, \tau^{j}\right) d y, \\
& \int_{\beta}\left(x^{j}, \varepsilon_{0}\right)
\end{aligned}
$$

Тогда имеет место следующее неравенство:

$$
\begin{aligned}
& \mid H\left(x^{1}, t, \tau^{1}\right)- H\left(x^{2}, t, \tau^{2}\right) \mid \leqslant \\
& \leqslant\left|H_{1}\left(x^{1}, t, \tau^{1}\right)\right|+\left|H_{1}\left(x^{2}, t, \tau^{2}\right)\right|+\left|H_{3}\left(x^{1}, t, \tau^{1}\right)\right|+\left|H_{3}\left(x^{2}, t, \tau^{2}\right)\right|+ \\
&+
\end{aligned}
$$

Пусть $R>0$ настолько велико, что $x^{1}, x^{2} \in O(0, R)$. Пусть $\varepsilon>0$ - произвольное фиксированное число. Тогда найдется настолько большое $R_{0}>0$, что в силу оценки (3.13) теоремы 3.1 будет выполнена следующая цепочка неравенств:

$$
\begin{aligned}
&\left|H_{1}\left(x^{j}, t, \tau^{j}\right)\right| \leqslant\left(1+R^{2}\right)^{1 / 2} \sup _{(y, \tau) \in \mathbb{R}^{3} \otimes[0, T]}|\rho(y, \tau)| \int_{\mathbb{R}^{3} \backslash O\left(x^{j}, R_{0}\right)} \frac{1}{\left(1+|y|^{2}\right)^{\beta}} \frac{K_{1}(T)}{\left|x^{j}-y\right|} d y \leqslant \\
& \leqslant\left(1+R^{2}\right)^{1 / 2} \sup _{(y, \tau) \in \mathbb{R}^{3} \otimes[0, T]}|\rho(y, \tau)| \frac{K_{1}(T)}{R_{0}} \int_{\mathbb{R}^{3}} \frac{1}{\left(1+|y|^{2}\right)^{\beta}} d y<\frac{\varepsilon}{5}
\end{aligned}
$$

при $\beta>3 / 2$. Теперь выберем настолько малое $\varepsilon_{0} \in\left(0, R_{0}\right)$, что в силу оценки (3.13) теоремы 3.1 будет выполнена следующая цепочка неравенств:

$$
\begin{aligned}
\left|H_{3}\left(x^{j}, t, \tau^{j}\right)\right| \leqslant\left(1+R^{2}\right)^{1 / 2} \sup _{(y, \tau) \in \mathbb{R}^{3} \otimes[0, T]}|\rho(y, \tau)| & \int_{O\left(x^{j}, \varepsilon_{0}\right)} \frac{K_{1}(T)}{\left|x^{j}-y\right|} d y= \\
& =\left(1+R^{2}\right)^{1 / 2} \sup _{(y, \tau) \in \mathbb{R}^{3} \otimes[0, T]}|\rho(y, \tau)| 4 \pi K_{1}(T)\left(\frac{\varepsilon_{0}}{2}\right)^{2}<\frac{\varepsilon}{5} .
\end{aligned}
$$

Теперь фиксируем найденные $R_{0}>\varepsilon_{0}>0$. Тогда найдется такое $\delta=\delta\left(\varepsilon_{0}, R_{0}, \varepsilon\right)>0$, что при

$$
\left|\left(x^{1}, \tau^{1}\right)-\left(x^{2}, \tau^{2}\right)\right|=\left(\sum_{j=1}^{3}\left(x_{j}^{1}-x_{j}^{2}\right)^{2}+\left(\tau^{1}-\tau^{2}\right)^{2}\right)^{1 / 2}<\delta
$$


будет справедлива следующая цепочка неравенств:

$$
\begin{aligned}
& \left|H_{2}\left(x^{1}, t, \tau^{1}\right)-H_{2}\left(x^{2}, t, \tau^{2}\right)\right| \leqslant \\
& \leqslant \int_{O\left(0, R_{0}\right) \backslash O\left(0, \varepsilon_{0}\right)} \mid \frac{\left(1+\left|x^{1}\right|^{2}\right)^{1 / 2} \rho\left(x^{1}+z, \tau^{1}\right)}{\left(1+\left|x^{1}+z\right|^{2}\right)^{\beta}} \mathscr{E}\left(z, t-\tau^{1}\right)- \\
& \quad-\frac{\left(1+\left|x^{2}\right|^{2}\right)^{1 / 2} \rho\left(x^{2}+z, \tau^{2}\right)}{\left(1+\left|x^{2}+z\right|^{2}\right)^{\beta}} \mathscr{E}\left(z, t-\tau^{2}\right) \mid d z<\frac{\varepsilon}{5}
\end{aligned}
$$

Таким образом, из (8.11)-(8.15) вытекает, что $H(x, t, \tau) \in \mathbb{C}\left(\mathbb{R}^{3} \otimes[0, t]\right)$. Докажем, что $H(x, t, \tau) \in$ $\mathbb{C}_{b}\left(\mathbb{R}^{3} \otimes[0, t]\right)$. С этой целью воспользуемся следующей оценкой:

$$
\sup _{(x, t) \in \mathbb{R}^{3} \otimes[0, T]} \int_{\mathbb{R}^{3}}\left|G_{\beta}(x, y, t)\right| d y \leqslant B_{3}(\beta, T)<+\infty,
$$

которая является следствием неравенства (3.29) теоремы 3.2. Поэтому из (8.16) вытекает следующая оценка:

$$
\sup _{(x, t, \tau) \in \mathbb{R}^{3} \otimes[0, T] \otimes[0, t]}|H(x, t, \tau)| \leqslant B_{11}(T)<+\infty \quad \text { для любого } t \in[0, T] .
$$

Таким образом, из (8.17) вытекает, что

$$
H(x, t, \tau) \in \mathbb{C}_{b}\left(\mathbb{R}^{3} \otimes[0, t]\right) \quad \text { для любого } t \in[0, T] .
$$

Шаг 2. Заметим, что с учетом теоремы из [1, раздел 1.6] и оценок (3.13) теоремы 3.1 справедливы поточечные равенства

$$
\begin{aligned}
& \frac{\partial H(x, t, \tau)}{\partial t}=\int_{\mathbb{R}^{3}} \frac{\partial G_{\beta}(x, y, t-\tau)}{\partial t} \rho(y, \tau) d y \\
& \frac{\partial H(x, t, \tau)}{\partial x_{j}}=\int_{\mathbb{R}^{3}} \frac{\partial G_{\beta}(x, y, t-\tau)}{\partial x_{j}} \rho(y, \tau) d y
\end{aligned}
$$

для всех $(x, t, \tau) \in \mathbb{R}^{3} \otimes[0, T] \otimes[0, t]$. Более того, с учетом оценок (3.13) точно так же, как при рассмотрении функции $H(x, t, \tau)$, можно доказать, что для каждого $t \in[0, T]$

$$
\frac{\partial H(x, t, \tau)}{\partial t} \in \mathbb{C}\left(\mathbb{R}^{3} \otimes[0, t]\right), \quad \frac{\partial H(x, t, \tau)}{\partial x_{j}} \in \mathbb{C}\left(\mathbb{R}^{3} \otimes[0, t]\right), \quad j=1,2,3 .
$$

Докажем, что на самом деле

$$
\frac{\partial H(x, t, \tau)}{\partial t} \in \mathbb{C}_{b}\left(\mathbb{R}^{3} \otimes[0, t]\right), \quad\left(1+|x|^{2}\right)^{1 / 2} \frac{\partial H(x, t, \tau)}{\partial x_{j}} \in \mathbb{C}_{b}\left(\mathbb{R}^{3} \otimes[0, t]\right)
$$

при $j=1,2,3$. Прежде всего заметим, что в силу оценки (3.29) из теоремы 3.2 имеет место неравенство

$$
\sup _{(x, t) \in \mathbb{R}^{3} \otimes[0, T]} \int_{\mathbb{R}^{3}}\left|\frac{\partial G_{\beta}(x, y, t)}{\partial t}\right| d y \leqslant B_{3}(\beta, T)<+\infty \quad \text { при } \quad \beta>\frac{3}{2} .
$$

С учетом оценки (8.23) из равенства (8.19) вытекает, что справедливо неравенство

$$
\sup _{(x, t, \tau) \in \mathbb{R}^{3} \otimes[0, T] \otimes[0, t]}\left|\frac{\partial H(x, t, \tau)}{\partial t}\right| \leqslant B_{1}(T, \beta) \sup _{(y, \tau) \in \mathbb{R}^{3} \otimes[0, T]}|\rho(y, \tau)|<+\infty,
$$

т.е доказано первое соотношение из (8.22). При $x \neq y$ справедливо следующее равенство:

$$
\left(1+|x|^{2}\right)^{1 / 2} \frac{\partial G_{\beta}(x, y, t)}{\partial x_{j}}=\frac{1+|x|^{2}}{\left(1+|y|^{2}\right)^{\beta}} \frac{\partial \mathscr{E}(x-y, t)}{\partial x_{j}}+\frac{x_{j}}{\left(1+|y|^{2}\right)^{\beta}} \mathscr{E}(x-y, t)
$$


С учетом оценок (3.13) из теоремы 3.1 имеет место неравенство

$$
\left|\left(1+|x|^{2}\right)^{1 / 2} \frac{\partial G_{\beta}(x, y, t)}{\partial x_{j}}\right| \leqslant \frac{1+|x|^{2}}{\left(1+|y|^{2}\right)^{\beta}} \frac{K_{1}(T)}{|x-y|^{2}}+\frac{\left(1+|x|^{2}\right)^{1 / 2}}{\left(1+|y|^{2}\right)^{\beta}} \frac{K_{1}(T)}{|x-y|} .
$$

Из этой оценки с учетом формул (3.30) вытекает неравенство

$$
\sup _{(x, t) \in \mathbb{R}^{3} \otimes[0, T]} \int\left|\left(1+|x|^{2}\right)^{1 / 2} \frac{\partial G_{\beta}(x, y, t)}{\partial x_{j}}\right| d y \leqslant B_{2}(T, \beta)<+\infty, \quad \beta>\frac{3}{2},
$$

из которого и из (8.20) получаем оценку

$$
\sup _{(x, t, \tau) \in \mathbb{R}^{3} \otimes[0, T] \otimes[0, t]}\left(1+|x|^{2}\right)^{1 / 2}\left|\frac{\partial H(x, t, \tau)}{\partial x_{j}}\right| \leqslant B_{2}(T, \beta) \sup _{(y, \tau) \in \mathbb{R}^{3} \otimes[0, T]}|\rho(y, \tau)|<+\infty .
$$

Итак, доказано второе соотношение из (8.22).

Аналогичным образом при помощи оценок (3.13) теоремы 3.1 с учетом формул (3.30) можно доказать, что

$$
\frac{\partial^{2} H(x, t, \tau)}{\partial x_{j} \partial t}=\frac{\partial^{2} H(x, t, \tau)}{\partial t \partial x_{j}} \in \mathbb{C}\left(\mathbb{R}^{3} \otimes[0, t]\right) \quad \text { для каждого } t \in[0, T]
$$

и следующую оценку:

$$
\sup _{(x, t, \tau) \in \mathbb{R}^{3} \otimes[0, T] \otimes[0, t]}\left(1+|x|^{2}\right)^{1 / 2}\left|\frac{\partial^{2} H(x, t, \tau)}{\partial t \partial x_{j}}\right| \leqslant B_{2}(T, \beta) \sup _{(y, \tau) \in \mathbb{R}^{3} \otimes[0, T]}|\rho(y, \tau)|<+\infty .
$$

Таким образом, из (8.5), (8.18) и (8.22) получаем, что для каждого $t \in[0, T]$

$$
U(x, t) \in \mathbb{C}_{b}^{1,0}\left(\left(1+|x|^{2}\right)^{1 / 2} ; \mathbb{R}^{3}\right) .
$$

Кроме того, справедливы равенства

$$
\frac{\partial U(x, t)}{\partial t}=H(x, t, t)+\int_{0}^{t} \frac{\partial H(x, t, \tau)}{\partial t} d \tau=\int_{0}^{t} \frac{\partial H(x, t, \tau)}{\partial t} d \tau \quad \text { для всех }(x, t) \in \mathbb{R}^{3} \otimes[0, T],
$$

из которых и из $(8.22),(8.30)$ вытекает следующая оценка:

$$
\sup _{t \in[0, T]}\left|\frac{\partial U(x, t)}{\partial t}\right|_{1} \leqslant T \sup _{(x, t, \tau) \in \mathbb{R}^{3} \otimes[0, T] \otimes[0, t]}\left|\frac{\partial H(x, t, \tau)}{\partial t}\right|_{1} \leqslant B_{3}(T, \beta)<+\infty \quad \text { при } \quad \beta>\frac{3}{2} .
$$

Заметим, что справедливо следующее равенство:

$$
U\left(x, t_{2}\right)-U\left(x, t_{1}\right)=\int_{t_{1}}^{t_{2}} \frac{\partial U(x, t)}{\partial t} d t
$$

из которого и из (8.33) вытекает следующая оценка:

$$
\left|U\left(x, t_{2}\right)-U\left(x, t_{1}\right)\right|_{1} \leqslant \sup _{t \in[0, T]}\left|\frac{\partial U(x, t)}{\partial t}\right|_{1}\left|t_{2}-t_{1}\right| \leqslant B_{3}(T, \beta)\left|t_{2}-t_{1}\right| .
$$

Из (8.31) и (8.35) приходим к выводу о том, что

$$
U(x, t) \in \mathbb{C}\left([0, T] ; \mathbb{C}_{b}^{1,0}\left(\left(1+|x|^{2}\right)^{1 / 2} ; \mathbb{R}^{3}\right)\right) \quad \text { при } \quad \beta>\frac{3}{2} .
$$

Шаг 3. Докажем теперь, что

$$
\frac{\partial U(x, t)}{\partial t} \in \mathbb{C}\left([0, T] ; \mathbb{C}_{b}^{1,0}\left(\left(1+|x|^{2}\right)^{1 / 2} ; \mathbb{R}^{3}\right)\right) \quad \text { при } \quad \beta>\frac{3}{2} .
$$

Прежде всего заметим, что при помощи оценок (3.13) и равенств (3.15) теоремы 3.1 можно доказать, что 


$$
\begin{aligned}
& \frac{\partial^{2} U(x, t)}{\partial t^{2}}=-\int_{\mathbb{R}^{3}} \frac{\left(1+|x|^{2}\right)^{1 / 2}}{\left(1+|y|^{2}\right)^{\beta}} \frac{\rho(y, t)}{|x-y|} d y+ \\
& \quad+\int_{0}^{t} \int_{\mathbb{R}^{3}} \frac{\partial^{2} G_{\beta}(x, y, t-\tau)}{\partial t^{2}} \rho(y, \tau) d y d \tau \in \mathbb{C}_{b}\left(\mathbb{R}^{3} \otimes[0, T]\right) .
\end{aligned}
$$

Действительно, это следствие оценки

$$
\sup _{(x, t) \in \mathbb{R}^{3} \otimes[0, T]} \int_{\mathbb{R}^{3}}\left|\frac{\partial^{2} G_{\beta}(x, y, t)}{\partial t^{2}}\right| d y \leqslant B_{3}(\beta, T)<+\infty \quad \text { при } \quad \beta>\frac{3}{2},
$$

справедливой в силу оценок $(3.29)$, а также того факта, что $\rho(x, t) \in \mathbb{C}\left([0, T] ; \mathbb{C}_{b}\left(\mathbb{R}^{3}\right)\right)$. Из теоремы в [1, раздел 1.6] и оценок (3.15) теоремы 3.1 вытекает, что

$$
\begin{aligned}
\frac{\partial^{3} U(x, t)}{\partial x_{j} \partial t^{2}}=-\int_{\mathbb{R}^{3}} \frac{\partial}{\partial x_{j}}\left(\frac{\left(1+|x|^{2}\right)^{1 / 2}}{\left(1+|y|^{2}\right)^{\beta}}\right. & \left.\frac{1}{|x-y|}\right) \rho(y, t) d y+ \\
& +\int_{0}^{t} \int_{\mathbb{R}^{3}} \frac{\partial^{3} G_{\beta}(x, y, t-\tau)}{\partial x_{j} \partial t^{2}} \rho(y, \tau) d y d \tau \in \mathbb{C}\left(\mathbb{R}^{3} \otimes[0, T]\right) .
\end{aligned}
$$

Из формул (3.30) получаем оценку

$$
\sup _{(x, t) \in \mathbb{R}^{3} \otimes[0, T]} \int_{\mathbb{R}^{3}}\left|\left(1+|x|^{2}\right)^{1 / 2} \frac{\partial^{3} G_{\beta}(x, y, t)}{\partial x_{j} \partial t^{2}}\right| d y \leqslant B_{3}(T, \beta)<+\infty \quad \text { при } \quad \beta>\frac{3}{2} .
$$

Кроме того, нетрудно получить оценку

$$
\sup _{x \in \mathbb{R}^{3}}\left(1+|x|^{2}\right)^{1 / 2} \int_{\mathbb{R}^{3}}\left|\frac{\partial}{\partial x_{j}}\left(\frac{\left(1+|x|^{2}\right)^{1 / 2}}{\left(1+|y|^{2}\right)^{\beta}} \frac{1}{|x-y|}\right)\right| d y=B_{4}(\beta)<+\infty \quad \text { при } \quad \beta>\frac{3}{2},
$$

поскольку при $x \neq y$ справедливо соотношение

$$
\left(1+|x|^{2}\right)^{1 / 2} \frac{\partial}{\partial x_{j}}\left(\frac{\left(1+|x|^{2}\right)^{1 / 2}}{\left(1+|y|^{2}\right)^{\beta}} \frac{1}{|x-y|}\right)=\frac{1}{\left(1+|y|^{2}\right)^{\beta}} \frac{x_{j}}{|x-y|}-\frac{1+|x|^{2}}{\left(1+|y|^{2}\right)^{\beta}} \frac{x_{j}-y_{j}}{|x-y|^{3}},
$$

из которого вытекает неравенство

$$
\begin{aligned}
\mid\left(1+|x|^{2}\right)^{1 / 2} \frac{\partial}{\partial x_{j}}\left(\frac{\left(1+|x|^{2}\right)^{1 / 2}}{\left(1+|y|^{2}\right)^{\beta}}\right. & \left.\frac{1}{|x-y|}\right) \mid \leqslant \\
& \leqslant \frac{\left(1+|x|^{2}\right)^{1 / 2}}{\left(1+|y|^{2}\right)^{\beta}} \frac{1}{|x-y|}+\frac{1+|x|^{2}}{\left(1+|y|^{2}\right)^{\beta}} \frac{1}{|x-y|^{2}} \quad \text { при } \quad x \neq y .
\end{aligned}
$$

Теперь осталось воспользоваться оценками (3.27) и (3.28) и доказать оценку (8.42). Таким образом, из (8.40) с учетом оценок (8.41) и (8.42) вытекает оценка

$$
\sup _{(x, t) \in \mathbb{R}^{3} \otimes[0, T]}\left|\left(1+|x|^{2}\right)^{1 / 2} \frac{\partial^{3} U(x, t)}{\partial x_{j} \partial t^{2}}\right| \leqslant B_{5}(\beta, T) \sup _{(y, \tau) \in \mathbb{R}^{3} \otimes[0, T]}|\rho(y, \tau)|<+\infty \quad \text { при } \quad \beta>\frac{3}{2} .
$$

Из (8.38) и (8.45) приходим к следующей оценке:

$$
\sup _{(x, t) \in \mathbb{R}^{3} \otimes[0, T]}\left|\frac{\partial^{2} U(x, t)}{\partial t^{2}}\right|_{1} \leqslant B_{6}(T, \beta) \sup _{(y, \tau) \in \mathbb{R}^{3} \otimes[0, T]}|\rho(y, \tau)|<+\infty \quad \text { при } \quad \beta>\frac{3}{2} .
$$

Заметим, что ранее (см. оценку (8.33)) было доказано, что

$$
\frac{\partial U(x, t)}{\partial t} \in \mathbb{C}_{b}^{1,0}\left(\left(1+|x|^{2}\right)^{1 / 2} ; \mathbb{R}^{3}\right) \quad \text { для всех } t \in[0, T] .
$$


Кроме того, справедливо равенство

$$
\frac{\partial U\left(x, t_{2}\right)}{\partial t}-\frac{\partial U\left(x, t_{1}\right)}{\partial t}=\int_{t_{1}}^{t_{2}} \frac{\partial^{2} U(x, t)}{\partial t^{2}} d t
$$

из которого с учетом оценки (8.46) вытекает оценка

$$
\left|\frac{\partial U\left(x, t_{2}\right)}{\partial t}-\frac{\partial U\left(x, t_{1}\right)}{\partial t}\right|_{1} \leqslant \int_{t_{1}}^{t_{2}}\left|\frac{\partial^{2} U(x, t)}{\partial t^{2}}\right|_{1} d t \leqslant B_{6}(T, \beta) \sup _{(y, \tau) \in \mathbb{R}^{3} \otimes[0, T]}|\rho(y, \tau)|\left|t_{2}-t_{1}\right| .
$$

Из (8.47) и (8.49) получаем, что

$$
\frac{\partial U(x, t)}{\partial t} \in \mathbb{C}\left([0, T] ; \mathbb{C}_{b}^{1,0}\left(\left(1+|x|^{2}\right)^{1 / 2} ; \mathbb{R}^{3}\right)\right) .
$$

Докажем, что сильная производная по времени от функции $U(x, t)$ в смысле банахова пространства $\mathbb{C}\left([0, T] ; \mathbb{C}_{b}^{1,0}\left(\left(1+|x|^{2}\right)^{1 / 2} ; \mathbb{R}^{3}\right)\right)$ совпадает с частной производной

$$
\frac{d U(x)(t)}{d t}=\frac{\partial U(x, t)}{\partial t} \in \mathbb{C}\left([0, T] ; \mathbb{C}_{b}^{1,0}\left(\left(1+|x|^{2}\right)^{1 / 2} ; \mathbb{R}^{3}\right)\right) .
$$

Прежде всего докажем, что справедливо поточечное равенство

$$
\frac{\partial^{2} U(x, t)}{\partial t \partial x_{j}}=\frac{\partial^{2} U(x, t)}{\partial x_{j} \partial t} \quad \text { для всех } \quad(x, t) \in \mathbb{R}^{3} \otimes[0, T] .
$$

Действительно, имеют место равенства

$$
\frac{\partial^{2} U(x, t)}{\partial t \partial x_{j}}=\int_{0}^{t} \frac{\partial^{2} H(x, t, \tau)}{\partial t \partial x_{j}} d \tau=\int_{0}^{t} \frac{\partial^{2} H(x, t, \tau)}{\partial x_{j} \partial t} d \tau=\frac{\partial^{2} U(x, t)}{\partial x_{j} \partial t},
$$

где мы воспользовались свойством коммутативности (8.29). Заметим, что справедливы соотношения

$$
\begin{aligned}
& \left|\frac{U(x)(t+\Delta t)-U(x)(t)}{\Delta t}-\frac{\partial U(x, t)}{\partial t}\right|_{1}= \\
& =\left|\frac{\partial U\left(x, t^{*}\right)}{\partial t}-\frac{\partial U(x, t)}{\partial t}\right|_{0}+\sum_{j=1}^{3}\left|\left(1+|x|^{2}\right)^{1 / 2}\left(\frac{\partial^{2} U\left(x, t^{*}\right)}{\partial t \partial x_{j}}-\frac{\partial U(x, t)}{\partial x_{j} \partial t}\right)\right|_{0} \leqslant \\
& \quad \leqslant \sup _{s \in[t, t+\Delta t]}\left|\frac{\partial U(x, s)}{\partial t}-\frac{\partial U(x, t)}{\partial t}\right|_{0}+ \\
& +\sup _{s \in[t, t+\Delta t]} \sum_{j=1}^{3}\left|\left(1+|x|^{2}\right)^{1 / 2}\left(\frac{\partial^{2} U(x, s)}{\partial t \partial x_{j}}-\frac{\partial U(x, t)}{\partial t \partial x_{j}}\right)\right|_{0} \rightarrow+0 \quad \text { при }|\Delta t| \rightarrow+0,
\end{aligned}
$$

где $t^{*}=t^{*}(x) \in(t, t+\Delta t)$, и мы воспользовались свойством (8.50), а также тем фактом, что если $f(x, t) \in \mathbb{C}\left([0, T] ; \mathbb{C}_{b}\left(\left(1+|x|^{2}\right)^{1 / 2} ; \mathbb{R}^{3}\right)\right)$, то

$$
g(s, t):=\sup _{x \in \mathbb{R}^{3}}|f(x, s)-f(x, t)|_{1} \in \mathbb{C}([0, T] \otimes[0, T])
$$

и поэтому

$$
\sup _{s \in[t, t+\Delta t]} g(s, t) \rightarrow+0 \quad \text { при }|\Delta t| \rightarrow+0 \text { для каждого } t \in[0, T] .
$$

Таким образом, на данный момент доказано, что

$$
U(x, t) \in \mathbb{C}^{(1)}\left([0, T] ; \mathbb{C}_{b}^{1,0}\left(\left(1+|x|^{2}\right)^{1 / 2} ; \mathbb{R}^{3}\right)\right) .
$$

Шаг 4. Теперь наша задача доказать, что

$$
U(x, t) \in \mathbb{C}^{(2)}\left([0, T] ; \mathbb{C}_{b}^{1,0}\left(\left(1+|x|^{2}\right)^{1 / 2} ; \mathbb{R}^{3}\right)\right) .
$$


С этой целью рассмотрим потенциал

$$
V_{0}(x, t):=-\int_{\mathbb{R}^{3}} \frac{\left(1+|x|^{2}\right)^{1 / 2}}{\left(1+|y|^{2}\right)^{\beta}} \frac{\rho(y, t)}{4 \pi|x-y|} d y .
$$

Лемма 8.1. Для любой функиии $\rho(x, t) \in \mathbb{C}\left([0, T] ; \mathbb{C}_{b}\left(\mathbb{R}^{3}\right)\right)$ потенииал $V_{0}(x, t)$ принадлежит классу $\mathbb{C}\left([0, T] ; \mathbb{C}_{b}^{1,0}\left(\left(1+|x|^{2}\right)^{1 / 2} ; \mathbb{R}^{3}\right)\right)$ при $\beta>3 / 2$.

Доказательство. При помощи оценок (3.26) и (3.27), справедливых при условии $\beta>3 / 2$, несложно доказать, что $V_{0}(x, t) \in \mathbb{C}_{b}^{1,0}\left(\left(1+|x|^{2}\right)^{1 / 2} ; \mathbb{R}^{3}\right)$ для каждого $t \in[0, T]$, причем справедлива оценка

$$
\left|V_{0}\left(x, t_{2}\right)-V_{0}\left(x, t_{1}\right)\right|_{1} \leqslant B_{7}(\beta)\left|\rho\left(x, t_{2}\right)-\rho\left(x, t_{1}\right)\right|_{0} \rightarrow+0 \quad \text { при } \quad\left|t_{2}-t_{1}\right| \rightarrow+0 .
$$

Лемма доказана.

Рассмотрим теперь следующий вспомогательный потенциал:

$$
U_{0}(x, t):=\int_{0}^{t} \int_{\mathbb{R}^{3}} \frac{\partial^{2} G_{\beta}(x, y, t-\tau)}{\partial t^{2}} \rho(y, \tau) d y d \tau .
$$

Лемма 8.2. Если функиия $\rho(x, t)$ принадлежит классу $\mathbb{C}\left([0, T] ; \mathbb{C}_{b}\left(\mathbb{R}^{3}\right)\right)$ и $\beta>3 / 2$, то потенииал $U_{0}(x, t)$ принадлежст классу $\mathbb{C}\left([0, T] ; \mathbb{C}_{b}^{1,0}\left(\left(1+|x|^{2}\right)^{1 / 2} ; \mathbb{R}^{3}\right)\right)$.

Доказательство. С учетом оценок (3.29) и (3.30) стандартным образом можно доказать, что для каждого $t \in[0, T]$ потенциал $U_{0}(x, t) \in \mathbb{C}_{b}^{1,0}\left(\left(1+|x|^{2}\right)^{1 / 2} ; \mathbb{R}^{3}\right)$. С учетом равенства (3.16) можно доказать равенство

$$
\begin{aligned}
\frac{\partial U_{0}(x, t)}{\partial t}=\int_{\mathbb{R}^{3}} & \frac{\partial^{2} G_{\beta}(x, y, 0)}{\partial t^{2}} \rho(y, t) d y+ \\
& \quad+\int_{0}^{t} \int_{\mathbb{R}^{3}} \frac{\partial^{3} G_{\beta}(x, y, t-\tau)}{\partial t^{3}} \rho(y, \tau) d y d \tau=\int_{0}^{t} \int_{\mathbb{R}^{3}} \frac{\partial^{3} G_{\beta}(x, y, t-\tau)}{\partial t^{3}} \rho(y, \tau) d y d \tau,
\end{aligned}
$$

причем с учетом оценки (3.29) заключаем, что

$$
\frac{\partial U_{0}(x, t)}{\partial t} \in \mathbb{C}_{b}\left(\mathbb{R}^{3} \otimes[0, T]\right) .
$$

Далее, используя оценки (3.29) и (3.30), можно получить оценку

$$
\left|\frac{\partial U_{0}(x, t)}{\partial t}\right|_{1} \leqslant B_{8}(T, \beta) \sup _{(y, \tau) \in \mathbb{R}^{3} \otimes[0, T]}|\rho(y, \tau)| \quad \text { при } \quad \beta>\frac{3}{2} .
$$

Таким образом, приходим к следующей оценке:

$$
\left|U_{0}\left(x, t_{2}\right)-U_{0}\left(x, t_{1}\right)\right|_{1} \leqslant \sup _{t \in[0, T]}\left|\frac{\partial U_{0}(x, t)}{\partial t}\right|_{1}\left|t_{2}-t_{1}\right| \leqslant B_{8}(T, \beta) \sup _{(y, \tau) \in \mathbb{R}^{3} \otimes[0, T]}|\rho(y, \tau)|\left|t_{2}-t_{1}\right| .
$$

Лемма доказана.

Таким образом, из (8.38) с учетом лемм 8.1 и 8.2 приходим к выводу о том, что

$$
\frac{\partial^{2} U(x, t)}{\partial t^{2}}=V_{0}(x, t)+U_{0}(x, t) \in \mathbb{C}\left([0, T] ; \mathbb{C}_{b}^{1,0}\left(\left(1+|x|^{2}\right)^{1 / 2} ; \mathbb{R}^{3}\right)\right)
$$

Нам осталось доказать, что

$$
\frac{d}{d t} \frac{\partial U(x, t)}{\partial t}=\frac{\partial^{2} U(x, t)}{\partial t^{2}}
$$


в смысле банахова пространства $\mathbb{C}\left([0, T] ; \mathbb{C}_{b}^{1,0}\left(\left(1+|x|^{2}\right)^{1 / 2} ; \mathbb{R}^{3}\right)\right)$. Прежде всего заметим, что точно так же, как равенство (8.52) (и с учетом этого равенства), можно доказать следующие коммутационные равенства:

$$
\frac{\partial^{3} U(x, t)}{\partial x_{j} \partial t \partial t}=\frac{\partial^{3} U(x, t)}{\partial t \partial x_{j} \partial t}=\frac{\partial^{3} U(x, t)}{\partial t \partial t \partial x_{j}} \quad \text { для всех } \quad(x, t) \in \mathbb{R}^{3} \otimes[0, T] .
$$

Тогда справедливы следующие соотношения:

$$
\begin{aligned}
& \left|\frac{1}{\Delta t}\left[\frac{\partial U(x, t+\Delta t)}{\partial t}-\frac{\partial U(x, t)}{\partial t}\right]-\frac{\partial^{2} U(x, t)}{\partial t^{2}}\right|_{1}= \\
& =\left|\frac{\partial^{2} U\left(x, t^{*}\right)}{\partial t^{2}}-\frac{\partial^{2} U(x, t)}{\partial t^{2}}\right|_{0}+\sum_{j=1}^{3}\left|\left(1+|x|^{2}\right)^{1 / 2}\left(\frac{\partial^{3} U\left(x, t^{*}\right)}{\partial t \partial x_{j} \partial t}-\frac{\partial^{3} U(x, t)}{\partial x_{j} \partial t^{2}}\right)\right|_{0} \leqslant \\
& \leqslant \sup _{s \in[t, t+\Delta t]}\left|\frac{\partial^{2} U(x, s)}{\partial t^{2}}-\frac{\partial^{2} U(x, t)}{\partial t^{2}}\right|_{0}+ \\
& \quad+\sup _{s \in[t, t+\Delta t]} \sum_{j=1}^{3}\left|\left(1+|x|^{2}\right)^{1 / 2}\left(\frac{\partial^{3} U(x, s)}{\partial t \partial x_{j} \partial t}-\frac{\partial^{3} U(x, t)}{\partial x_{j} \partial t^{2}}\right)\right|_{0} \rightarrow+0
\end{aligned}
$$

при $|\Delta t| \rightarrow+0$, где $t^{*}=t^{*}(x) \in(t, t+\Delta t)$, и мы воспользовались коммутационными соотношениями (8.66) и свойством (8.64). Таким образом, вложение (8.56) доказано.

Рассмотрим теперь следующие два объемных потенциала:

$$
\begin{aligned}
& U_{0}^{(1)}(x, t)=\int_{0}^{t} \int_{\mathbb{R}^{3}} \frac{\partial G_{\beta}(x, y, t-\tau)}{\partial t} \rho(y, \tau) d y d \tau, \\
& U_{0}^{(2)}(x, t)=\int_{0}^{t} \int_{\mathbb{R}^{3}} \frac{\partial^{2} G_{\beta}(x, y, t-\tau)}{\partial t^{2}} \rho(y, \tau) d y d \tau,
\end{aligned}
$$

где функция $G_{\beta}(x, y, t)$ определена равенством (8.4). При доказательстве теоремы 8.1 фактически было доказано следующее утверждение.

Теорема 8.2. Если $\beta>3 / 2$ u $\rho(x, t) \in \mathbb{C}\left([0, T] ; \mathbb{C}_{b}\left(\mathbb{R}^{3}\right)\right)$, mo

$$
\begin{gathered}
U_{0}^{(1)}(x, t) \in \mathbb{C}^{(1)}\left([0, T] ; \mathbb{C}_{b}^{1,0}\left(\left(1+|x|^{2}\right)^{1 / 2} ; \mathbb{R}^{3}\right)\right), \\
U_{0}^{(2)}(x, t) \in \mathbb{C}\left([0, T] ; \mathbb{C}_{b}^{1,0}\left(\left(1+|x|^{2}\right)^{1 / 2} ; \mathbb{R}^{3}\right)\right) .
\end{gathered}
$$

Теорема 8.3. Пусть $\mu(x), \sigma(x) \in \mathbb{C}_{b}\left(\mathbb{R}^{3}\right)$ и $\beta>3 / 2$. Тогда для произвольного $T>0$ поверхностные потенииалы $V(x, t), W(x, t)$ принадлежсат классу $\mathbb{C}^{(2)}\left([0, T] ; \mathbb{C}_{b}^{1,0}\left(\left(1+|x|^{2}\right)^{1 / 2} ; \mathbb{R}^{3}\right)\right)$.

Доказательство. Доказательство основано на той же технике, что и доказательство теоремы 8.1. При этом существенно используются оценки (3.29), (3.30) теоремы 3.2.

Сформулируем и докажем теорему о свойствах объемного и поверхностных потенциалов (без веса) следующего вида:

$$
\begin{aligned}
U^{1}(x, t) & :=\int_{0}^{t} \int_{\mathbb{R}^{3}} \mathscr{E}(x-y, t-\tau) \rho_{1}(y, \tau) d y d \tau, \\
V^{1}(x, t) & :=\int_{\mathbb{R}^{3}} \mathscr{E}(x-y, t) \mu_{1}(y) d y \\
W^{1}(x, t) & :=\int_{\mathbb{R}^{3}} \frac{\mathscr{E}(x-y, t)}{\partial t} \sigma_{1}(y) d y
\end{aligned}
$$


где $\mathscr{E}(x, t)$ - фундаментальное решение, определенное равенством (3.12). Справедлива следующая теорема о гладкости этих потенциалов.

Теорема 8.4. Пусть $\rho_{1}(x, t) \in \mathbb{C}\left([0, T] ; \mathbb{C}_{b}\left(\left(1+|x|^{2}\right)^{\beta} ; \mathbb{R}^{3}\right)\right) u \mu_{1}(x), \sigma_{1}(x) \in \mathbb{C}_{b}\left(\left(1+|x|^{2}\right)^{\beta} ; \mathbb{R}^{3}\right)$. Тогда при $\beta>3 / 2$

$$
\begin{gathered}
U^{1}(x, t) \in \mathbb{C}^{(2)}\left([0, T] ; \mathbb{C}_{b}^{1,1 / 2}\left(\left(1+|x|^{2}\right) ; \mathbb{R}^{3}\right)\right), \\
V^{1}(x, t), W^{1}(x, t) \in \mathbb{C}^{(2)}\left(\left[0, T_{1}\right] ; \mathbb{C}_{b}^{1,1 / 2}\left(\left(1+|x|^{2}\right) ; \mathbb{R}^{3}\right)\right) \quad \text { для любого } \quad T_{1}>0 .
\end{gathered}
$$

Доказательство. Заметим, что справедливы равенства

$$
U^{1}(x, t)=\frac{U(x, t)}{\left(1+|x|^{2}\right)^{1 / 2}}, \quad V^{1}(x, t)=\frac{V(x, t)}{\left(1+|x|^{2}\right)^{1 / 2}}, \quad W^{1}(x, t)=\frac{W(x, t)}{\left(1+|x|^{2}\right)^{1 / 2}},
$$

в которых

$$
\rho(x, t)=\left(1+|x|^{2}\right)^{\beta} \rho_{1}(x, t), \quad \mu(x)=\left(1+|x|^{2}\right)^{\beta} \mu_{1}(x), \quad \sigma(x)=\left(1+|x|^{2}\right)^{\beta} \sigma_{1}(x) .
$$

Осталось воспользоваться теоремами 8.1 и 8.3, а также определениями банаховых пространств $\mathbb{C}_{b}^{1,0}\left(\left(1+|x|^{2}\right)^{1 / 2} ; \mathbb{R}^{3}\right)$ и $\mathbb{C}_{b}^{1,1 / 2}\left(\left(1+|x|^{2}\right) ; \mathbb{R}^{3}\right)$. Теорема доказана.

Справедлива следующая лемма (доказательство см., например, в [10]).

Лемма 8.3. Пусть $\rho_{0}(x) \in \mathbb{C}_{b}\left(\left(1+|x|^{2}\right)^{\beta} ; \mathbb{R}^{3}\right)$ nри $\beta>3 / 2$. Тогда классический обдемный нвютоновский потенциал

$$
W_{0}(x)=-\int_{\mathbb{R}^{3}} \frac{1}{4 \pi|x-y|} \rho_{0}(y) d y
$$

удовлетворяет равенству

$$
\left\langle\Delta_{x} W_{0}(x), \phi(x)\right\rangle=\left\langle\rho_{0}(x), \phi(x)\right\rangle \quad \text { для каждого } t \in[0, T]
$$

и для любых $\phi(x) \in \mathscr{D}\left(\mathbb{R}^{3}\right)$, где $\langle\cdot, \cdot\rangle$ - скобки двойственности между $\mathscr{D}\left(\mathbb{R}^{3}\right)$ и $\mathscr{D}^{\prime}\left(\mathbb{R}^{3}\right)$, оператор $\Delta_{x}$ - трехмерный оператор Лапласа, понимаемый в смысле производных обобщенных функиий.

Приступим к доказательству ряда утверждений относительно объемного и поверхностных потенциалов, определенных равенствами (8.70) и (8.71), (8.72).

Лемма 8.4. Пусть $\rho_{1}(x, t) \in \mathbb{C}\left([0, T] ; \mathbb{C}_{b}\left(\left(1+|x|^{2}\right)^{\beta} ; \mathbb{R}^{3}\right)\right)$ nри $\beta>3 / 2$. Тогда справедливо равенство

$$
\left\langle\mathfrak{M}_{x, t}\left[U^{1}\right](x, t), \phi(x)\right\rangle=\left\langle\rho_{1}(x, t), \phi(x)\right\rangle \quad \text { для всех } t \in[0, T]
$$

и для всех $\phi(x) \in \mathscr{D}\left(\mathbb{R}^{3}\right)$, где $\langle\cdot, \cdot\rangle$ - скобки двойственности между $\mathscr{D}\left(\mathbb{R}^{3}\right)$ и $\mathscr{D}^{\prime}\left(\mathbb{R}^{3}\right)$, оператор $\mathfrak{M}_{x, t}$ определен равенством

$$
\mathfrak{M}_{x, t}[w](x, t):=\Delta_{x} \frac{\partial^{2} w(x, t)}{\partial t^{2}}+\sum_{j=1}^{3} \omega_{j}^{2} w_{x_{j} x_{j}}(x, t),
$$

в котором производные по координатам понимаются в смысле обобщенных функций из $\mathscr{D}^{\prime}\left(\mathbb{R}^{3}\right)$. Кроме того,

$$
U^{1}(x, 0)=\frac{\partial U^{1}}{\partial t}(x, 0)=0 \quad \text { для всех } x \in \mathbb{R}^{3} .
$$

Доказательство. В силу теорем 3.1 и 8.4 справедливо следующее поточечное равенство:

$$
\begin{aligned}
& \frac{\partial^{2} U^{1}(x, t)}{\partial t^{2}}=-\int_{\mathbb{R}^{3}} \frac{1}{4 \pi|x-y|} \rho_{1}(y, t) d y+ \\
& \quad+\int_{0}^{t} \int_{\mathbb{R}^{3}} \mathscr{E}_{2}(x-y, t-\tau) \rho_{1}(y, \tau) d y d \tau=: W_{0}(x, t)+U^{2}(x, t),
\end{aligned}
$$


где

$$
\mathscr{E}_{2}(x-y, t-\tau):=\frac{\partial^{2} \mathscr{E}(x-y, t-\tau)}{\partial t^{2}} .
$$

В силу леммы 8.3 справедливо равенство

$$
\left\langle\Delta_{x} W_{0}(x, t), \phi(x)\right\rangle=\left\langle\rho_{1}(x, t), \phi(x)\right\rangle \quad \text { для каждого } t \in[0, T]
$$

и для любых пробных функций $\phi(x) \in \mathscr{D}\left(\mathbb{R}^{3}\right)$. С учетом введенных обозначений справедливо следующее равенство:

$$
\begin{aligned}
&\left\langle\Delta_{x} U^{2}(x, t)+\sum_{j=1}^{3} \omega_{j}^{2} U_{x_{j} x_{j}}^{1}(x, t), \phi(x)\right\rangle= \\
&=\left\langle U^{2}(x, t), \Delta_{x} \phi(x)\right\rangle+\sum_{j=1}^{3} \omega_{j}^{2}\left\langle U^{1}(x, t), \phi_{x_{j} x_{j}}(x)\right\rangle:=J_{1}+J_{2}
\end{aligned}
$$

для любой $\phi(x) \in \mathscr{D}\left(\mathbb{R}^{3}\right)$. Теперь фиксируем произвольным образом пробную функцию $\phi(x) \in$ $\mathscr{D}\left(\mathbb{R}^{3}\right)$. Найдется такое число $R=R(\phi)>0$, что $\operatorname{supp} \phi(x) \subset O(0, R)$. Рассмотрим сначала слагаемое $J_{1}$ из равенства (8.85). Справедливы равенства

$$
\begin{aligned}
J_{1}=\int_{O(0, R)} \Delta_{x} \phi(x) & \int_{0}^{t} \int_{\mathbb{R}^{3}} \mathscr{E}_{2}(x-y, t-\tau) \rho_{1}(y, \tau) d y d \tau d x= \\
& =\int_{0}^{t} \int_{O(0, R)} \Delta_{x} \phi(x) \int_{O(0,2 R)} \mathscr{E}_{2}(x-y, t-\tau) \rho_{1}(y, \tau) d y d x d \tau+ \\
& +\int_{0}^{t} \int_{O(0, R)} \Delta_{x} \phi(x) \int_{\mathbb{R}^{3} \backslash O(0,2 R)} \mathscr{E}_{2}(x-y, t-\tau) \rho_{1}(y, \tau) d y d x d \tau:=J_{11}+J_{12} .
\end{aligned}
$$

Заметим, что при $|y| \geqslant 2 R$ и $|x| \leqslant R$ справедливо неравенство

$$
|y-x| \geqslant|y|-|x| \geqslant 2 R-R=R>0 .
$$

Интегрируя по частям в выражении для $J_{12}$, получим равенство

$$
J_{12}=\int_{0}^{t} \int_{O(0, R)} \phi(x) \int_{\mathbb{R}^{3} \backslash O(0,2 R)} \Delta_{x} \mathscr{E}_{2}(x-y, t-\tau) \rho_{1}(y, \tau) d y d x d \tau .
$$

Рассмотрим теперь выражение для $J_{2}$ из формулы (8.85). Справедлива следующая цепочка равенств:

$$
\begin{aligned}
& J_{2}=\sum_{j=1}^{3} \omega_{j}^{2}\left\langle U^{1}(x, t), \phi_{x_{j} x_{j}}(x)\right\rangle= \\
&=\int_{O(0, R)} d x \sum_{j=1}^{3} \omega_{j}^{2} \phi_{x_{j} x_{j}}(x) \int_{0}^{t} \int_{\mathbb{R}^{3}} \mathscr{E}(x-y, t-\tau) \rho_{1}(y, \tau) d y d \tau d x= \\
&=\int_{O(0, R)} d x \sum_{j=1}^{3} \omega_{j}^{2} \phi_{x_{j} x_{j}}(x) \int_{0}^{t} \int_{O(0,2 R)} \mathscr{E}(x-y, t-\tau) \rho_{1}(y, \tau) d y d \tau d x+ \\
&+\int_{O(0, R)} d x \sum_{j=1}^{3} \omega_{j}^{2} \phi_{x_{j} x_{j}}(x) \int_{0}^{t} \int_{\mathbb{R}^{3} \backslash O(0,2 R)} \mathscr{E}(x-y, t-\tau) \rho_{1}(y, \tau) d y d \tau d x:=J_{21}+J_{22} .
\end{aligned}
$$


В силу (8.87) интегрированием по частям в выражении для $J_{22}$ можно получить равенство

$$
J_{22}=\int_{0}^{t} \int_{O(0, R)} \phi(x) \int_{\mathbb{R}^{3} \backslash O(0,2 R)} \sum_{j=1}^{3} \omega_{j}^{2} \mathscr{E}_{x_{j} x_{j}}(x-y, t-\tau) \rho_{1}(y, \tau) d y d x d \tau .
$$

Из выражений $(8.88),(8.90)$ и определения фундаментального решения $\mathscr{E}(x, t)$ получаем

$$
\begin{array}{r}
J_{12}+J_{22}=\int_{0}^{t} \int_{O(0, R)} \phi(x) \int_{\mathbb{R}^{3} \backslash O(0,2 R)}\left[\Delta_{x} \mathscr{E}_{2}(x-y, t-\tau)+\sum_{j=1}^{3} \omega_{j}^{2} \mathscr{E}_{x_{j} x_{j}}(x-y, t-\tau)\right] \times \\
\times \rho_{1}(y, \tau) d y d x d \tau=0 .
\end{array}
$$

Из равенств (8.86) и (8.89) вытекает следующая цепочка равенств:

$$
\begin{aligned}
& J_{11}+J_{21}=\int_{O(0, R)} \Delta_{x} \phi(x) \int_{0}^{t} \int_{O(0,2 R)} \mathscr{E}_{2}(x-y, t-\tau) \rho_{1}(y, \tau) d y d x d \tau+ \\
& \quad+\int_{O(0, R)} d x \sum_{j=1}^{3} \omega_{j}^{2} \phi_{x_{j} x_{j}}(x) \int_{0}^{t} \int_{O(0,2 R)} \mathscr{E}(x-y, t-\tau) \rho_{1}(y, \tau) d y d \tau d x= \\
& =\int_{0}^{t} \int_{O(0,2 R)} \rho_{1}(y, \tau) \int_{O(0,3 R)}\left[\frac{\partial^{2} \mathscr{E}(x-y, t-\tau)}{\partial t^{2}} \Delta_{x} \phi(x)+\mathscr{E}(x-y, t-\tau) \sum_{j=1}^{3} \omega_{j}^{2} \phi_{x_{j} x_{j}}(x)\right] d x d y d \tau,
\end{aligned}
$$

где последнее равенство имеет место, поскольку $\operatorname{supp} \phi(x) \subset O(0, R) \subset O(0,3 R)$. Отдельно рассмотрим интеграл

$$
K:=\int_{O(0,3 R)}\left[\frac{\partial^{2} \mathscr{E}(x-y, t-\tau)}{\partial t^{2}} \Delta_{x} \phi(x)+\mathscr{E}(x-y, t-\tau) \sum_{j=1}^{3} \omega_{j}^{2} \phi_{x_{j} x_{j}}(x)\right] d x d y d \tau,
$$

при $y \in O(0,2 R)$. В силу оценок (3.13) справедливо предельное равенство

$$
K=\lim _{\varepsilon \rightarrow+0} K^{\varepsilon},
$$

где

$$
K^{\varepsilon}:=\int_{O(0,3 R) \backslash O(y, \varepsilon)}\left[\frac{\partial^{2} \mathscr{E}(x-y, t-\tau)}{\partial t^{2}} \Delta_{x} \phi(x)+\mathscr{E}(x-y, t-\tau) \sum_{j=1}^{3} \omega_{j}^{2} \phi_{x_{j} x_{j}}(x)\right] d x d y d \tau,
$$

при $\varepsilon \in(0, R / 2)$ и для любого $y \in O(0,2 R)$. Теперь мы проведем интегрирование по частям в выражении (8.95) и получим равенство

$$
K^{\varepsilon}=K_{1}^{\varepsilon}+K_{2}^{\varepsilon}+K_{3}^{\varepsilon},
$$

где

$$
\begin{aligned}
& K_{1}^{\varepsilon}=\int_{\partial O(0,3 R) \cup \partial O(y, \varepsilon)}\left[\frac{\partial^{2} \mathscr{E}(x-y, t-\tau)}{\partial t^{2}} \frac{\partial \phi(x)}{\partial n_{x}}+\mathscr{E}(x-y, t-\tau) \sum_{j=1}^{3} \omega_{j}^{2} \frac{\partial \phi(x)}{\partial x_{j}} \cos \left(n_{x}, e_{j}\right)\right] d S_{x}, \\
& K_{2}^{\varepsilon}=-\int_{\partial O(0,3 R) \cup \partial O(y, \varepsilon)}\left[\frac{\partial^{3} \mathscr{E}(x-y, t-\tau)}{\partial t^{2} \partial n_{x}}+\sum_{j=1}^{3} \omega_{j}^{2} \frac{\partial \mathscr{E}(x-y, t-\tau)}{\partial x_{j}} \cos \left(n_{x}, e_{j}\right)\right] \phi(x) d S_{x},
\end{aligned}
$$




$$
K_{3}^{\varepsilon}=\int_{O(0,3 R) \backslash O(y, \varepsilon)} \phi(x) \mathfrak{M}_{x, t}[\mathscr{E}](x-y, t-\tau) d x=0,
$$

поскольку согласно определению фундаментального решения $\mathscr{E}(x-y, t-\tau)$ имеет место равенство

$$
\mathfrak{M}_{x, t}[\mathscr{E}](x-y, t)=0 \quad \text { для всех } \quad(x, t) \in(O(0,3 R) \backslash O(y, \varepsilon)) \times[0, T] .
$$

Кроме того,

$$
\int_{\partial O(0,3 R)}\left[\frac{\partial^{2} \mathscr{E}(x-y, t-\tau)}{\partial t^{2}} \frac{\partial \phi(x)}{\partial n_{x}}++\mathscr{E}(x-y, t-\tau) \sum_{j=1}^{3} \omega_{j}^{2} \frac{\partial \phi(x)}{\partial x_{j}} \cos \left(n_{x}, e_{j}\right)\right] d S_{x}=0
$$

поскольку $\operatorname{supp} \phi(x) \subset O(0, R)$. Далее,

$$
\int_{\partial O(y, \varepsilon)}\left[\frac{\partial^{2} \mathscr{E}(x-y, t-\tau)}{\partial t^{2}} \frac{\partial \phi(x)}{\partial n_{x}}+\mathscr{E}(x-y, t-\tau) \sum_{j=1}^{3} \omega_{j}^{2} \frac{\partial \phi(x)}{\partial x_{j}} \cos \left(n_{x}, e_{j}\right)\right] d S_{x} \rightarrow 0
$$

при $\varepsilon \rightarrow+0$ для каждого фиксированного $y \in O(0,2 R)$, поскольку $\phi(x) \in \mathbb{C}_{0}^{\infty}(O(0,3 R))$ и справедливы оценки (3.13) фундаментального решения $\mathscr{E}(x, t)$, а площадь сферы $\partial O(y, \varepsilon)$ равна $2 \pi \varepsilon^{2}$. Следовательно, имеем

$$
\lim _{\varepsilon \rightarrow+0} K_{1}^{\varepsilon}=0 .
$$

Наконец, поскольку $\phi(x)=0$ на $\partial O(0,3 R)$, то от выражения для $K_{2}^{\varepsilon}$ остается только следующий интеграл:

$$
K_{2}^{\varepsilon}=-\int_{\partial O(y, \varepsilon)}\left[\frac{\partial^{3} \mathscr{E}(x-y, t-\tau)}{\partial t^{2} \partial n_{x}}+\sum_{j=1}^{3} \omega_{j}^{2} \frac{\partial \mathscr{E}(x-y, t-\tau)}{\partial x_{j}} \cos \left(n_{x}, e_{j}\right)\right] \phi(x) d S_{x},
$$

который перепишем в виде

$$
\begin{aligned}
& K_{2}^{\varepsilon}=-\phi(y) \int_{\partial O(y, \varepsilon)}\left[\frac{\partial^{3} \mathscr{E}(x-y, t-\tau)}{\partial t^{2} \partial n_{x}}+\sum_{j=1}^{3} \omega_{j}^{2} \frac{\partial \mathscr{E}(x-y, t-\tau)}{\partial x_{j}} \cos \left(n_{x}, e_{j}\right)\right] d S_{x}+ \\
&+\int_{\partial O(y, \varepsilon)}\left[\frac{\partial^{3} \mathscr{E}(x-y, t-\tau)}{\partial t^{2} \partial n_{x}}+\sum_{j=1}^{3} \omega_{j}^{2} \frac{\partial \mathscr{E}(x-y, t-\tau)}{\partial x_{j}} \cos \left(n_{x}, e_{j}\right)\right][\phi(y)-\phi(x)] d S_{x}= \\
&:=K_{21}^{\varepsilon}+K_{22}^{\varepsilon}
\end{aligned}
$$

Заметим, что

$$
|\phi(x)-\phi(y)| \leqslant a(y, \varepsilon)|x-y| \quad \text { для всех } \quad x \in O(y, \varepsilon),
$$

и поэтому в силу оценок (3.13) фундаментального решения $\mathscr{E}(x, t)$ приходим к предельному свойству

$$
\lim _{\varepsilon \rightarrow+0} K_{22}^{\varepsilon}=0
$$

Осталось заметить, что

$$
\int_{\partial O(y, \varepsilon)}\left[\frac{\partial^{3} \mathscr{E}(x-y, t-\tau)}{\partial t^{2} \partial n_{x}}+\sum_{j=1}^{3} \omega_{j}^{2} \frac{\partial \mathscr{E}(x-y, t-\tau)}{\partial x_{j}} \cos \left(n_{x}, e_{j}\right)\right] d S_{x}=0
$$

силу полученного ранее равенства (5.18) леммы 5.2. Таким образом, из (8.104)-(8.106) вытекает предельное свойство

$$
\lim _{\varepsilon \rightarrow+0} K_{2}^{\varepsilon}=0 .
$$


Следовательно, из (8.96), (8.102) и (8.107) выводим, что $K=0$. Поэтому из (8.92) и (8.93) следует, что $J_{11}+J_{21}=0$. Отсюда и из $(8.85)-(8.92)$ получаем, что

$$
\left\langle\Delta_{x} U^{2}(x, t)+\sum_{j=1}^{3} \omega_{j}^{2} U_{x_{j} x_{j}}^{1}(x, t), \phi(x)\right\rangle=0
$$

для любой $\phi(x) \in \mathscr{D}\left(\mathbb{R}^{3}\right)$. Отсюда и из (8.84) с учетом (8.82) вытекает утверждение леммы.

Аналогичным образом доказывается следующее утверждение.

Лемма 8.5. Пусть $\mu_{1}(x), \sigma_{1}(x) \in \mathbb{C}_{b}\left(\left(1+|x|^{2}\right)^{\beta} ; \mathbb{R}^{3}\right)$ при $\beta>3 / 2$. Тогда справедливы следующие равенства:

$$
\left\langle\mathfrak{M}_{x, t}\left[V^{1}\right](x, t), \phi(x)\right\rangle=0, \quad\left\langle\mathfrak{M}_{x, t}\left[W^{1}\right](x, t), \phi(x)\right\rangle=0 \quad n p u \quad t \in[0,+\infty)
$$

и для всех $\phi(x) \in \mathscr{D}\left(\mathbb{R}^{3}\right)$, а оператор $\mathfrak{M}_{x, t}$ определен равенством (8.80).

Лемма 8.6. Пусть $u_{0}(x), u_{1}(x) \in \mathbb{C}_{b}^{(2)}\left(\left(1+|x|^{2}\right)^{\beta} ; \mathbb{R}^{3}\right)$. Тогда справедливы следующие равенства:

$$
\begin{aligned}
& W^{1}\left[\Delta u_{0}\right](x, 0)=-\int_{\mathbb{R}^{3}} \frac{1}{4 \pi|x-y|} \Delta u_{0}(y) d y=u_{0}(x), \\
& \frac{\partial V^{1}\left[\Delta u_{1}\right]}{\partial t}(x, 0)=-\int_{\mathbb{R}^{3}} \frac{1}{4 \pi|x-y|} \Delta u_{1}(y) d y=u_{1}(x)
\end{aligned}
$$

для всех $x \in \mathbb{R}^{3}$. Кроме того, для всех $\mu_{1}(x), \sigma_{1}(x) \in \mathbb{C}_{b}\left(\left(1+|x|^{2}\right)^{\beta} ; \mathbb{R}^{3}\right)$ при $\beta>3 / 2$ справедливы следующие равенства:

$$
V^{1}\left[\mu_{1}\right](x, 0)=0, \quad \frac{\partial W^{1}\left[\sigma_{1}\right]}{\partial t}(x, 0)=0 \quad \text { для всех } x \in \mathbb{R}^{3} .
$$

Доказательство. Равенства (8.110) и (8.111) доказаны в [10]. Равенства (8.112) являются следствиями свойств фундаментального решения (3.16).

Введем обозначение

$$
L(x, t):=U^{1}\left[\rho_{1}\right](x, t)+V^{1}\left[\Delta u_{1}\right](x, t)+W^{1}\left[\Delta u_{0}\right](x, t) .
$$

Из теоремы 8.4 и лемм 8.4-8.6 вытекает следующее утверждение.

Теорема 8.5. Пусть $\rho_{1}(x, t) \in \mathbb{C}\left([0, T] ; \mathbb{C}_{b}\left(\left(1+|x|^{2}\right)^{\beta} ; \mathbb{R}^{3}\right)\right), u_{0}(x) \in \mathbb{C}_{b}^{(2)}\left(\left(1+|x|^{2}\right)^{\beta_{1}} ; \mathbb{R}^{3}\right) u$ $u_{1}(x) \in \mathbb{C}_{b}^{(2)}\left(\left(1+|x|^{2}\right)^{\beta_{2}} ; \mathbb{R}^{3}\right)$ nри $\beta>3 / 2, \beta_{1}>3 / 2 u \beta_{2}>3 / 2$. Тогда

$$
L(x, t) \in \mathbb{C}^{(2)}\left([0, T] ; \mathbb{C}_{b}^{1,1 / 2}\left(\left(1+|x|^{2}\right) ; \mathbb{R}^{3}\right)\right),
$$

справедливо равенство

$$
\left\langle\mathfrak{M}_{x, t}[L](x, t), \phi(x)\right\rangle=\left\langle\rho_{1}(x, t), \phi(x)\right\rangle \quad \text { для всех } t \in[0, T]
$$

и для любых $\phi(x) \in \mathscr{D}\left(\mathbb{R}^{3}\right)$, где $\langle\cdot, \cdot\rangle$ - скобки двойственности между $\mathscr{D}\left(\mathbb{R}^{3}\right)$ и $\mathscr{D}^{\prime}\left(\mathbb{R}^{3}\right)$, а оператор $\mathfrak{M}_{x, t}$ определен равенством

$$
\mathfrak{M}_{x, t}[w](x, t):=\Delta_{x} \frac{\partial^{2} w(x, t)}{\partial t^{2}}+\sum_{j=1}^{3} \omega_{j}^{2} w_{x_{j} x_{j}}(x, t),
$$

в котором производные по координатам понимаются в смысле обобщенных функиий из $\mathscr{D}^{\prime}\left(\mathbb{R}^{3}\right)$. Кроме того, выполнены следующие начальные условия:

$$
L(x, 0)=u_{0}(x), \quad \frac{\partial L}{\partial t}(x, 0)=u_{1}(x) \quad \text { для всех } x \in \mathbb{R}^{3} .
$$




\section{9. ЗАДАЧА КОШИ (7.1), (7.2): СУЩЕСТВОВАНИЕ ЛОКАЛЬНЫХ} И ОТСУТСТВИЕ ЛОКАЛЬНЫХ И ГЛОБАЛЬНЫХ ВО ВРЕМЕНИ СЛАБЫХ РЕШЕНИЙ

Рассмотрим следующее вспомогательное интегральное уравнение:

$$
\begin{aligned}
u(x, t)=-\int_{0}^{t} \int_{\mathbb{R}^{3}} \mathscr{E}(x-y, t-\tau)|\nabla u(y, \tau)|^{q} d y d \tau+ & \\
& +\int_{\mathbb{R}^{3}} \frac{\partial \mathscr{E}(x-y, t)}{\partial t} \Delta u_{0}(y) d y+\int_{\mathbb{R}^{3}} \mathscr{E}(x-y, t) \Delta u_{1}(y) d y .
\end{aligned}
$$

Перейдем в этом интегральном уравнении к новой функции

$$
v(x, t)=\left(1+|x|^{2}\right)^{1 / 2} u(x, t)
$$

и учтем следующее равенство в классе дифференцируемых функций:

$$
\begin{aligned}
|\nabla u(x, t)|^{q}=\left|\nabla \frac{v(x, t)}{\left(1+|x|^{2}\right)^{1 / 2}}\right|^{q}= & \left|\frac{1}{\left(1+|x|^{2}\right)^{1 / 2}} \nabla v(x, t)-\frac{x}{\left(1+|x|^{2}\right)^{3 / 2}} v(x, t)\right|^{q}= \\
& =\frac{1}{\left(1+|x|^{2}\right)^{q}}\left|\left(1+|x|^{2}\right)^{1 / 2} \nabla v(x, t)-\frac{x}{\left(1+|x|^{2}\right)^{1 / 2}} v(x, t)\right|^{q} .
\end{aligned}
$$

После подстановки выражений (9.2) и (9.3) в интегральное уравнение (9.1) получим следующее интегральное уравнение относительно новой функции $v(x, t)$ :

$$
v(x, t)=\int_{0}^{t} \int_{\mathbb{R}^{3}} G_{q}(x, y, t-\tau) \rho(y, \tau) d y d \tau+\int_{\mathbb{R}^{3}} \frac{\partial G_{\alpha}(x, y, t)}{\partial t} \mu_{\alpha}(y) d y+\int_{\mathbb{R}^{3}} G_{\beta}(x, y, t) \sigma_{\beta}(y) d y,
$$

где

$$
\begin{gathered}
G_{\gamma}(x, y, t):=\frac{\left(1+|x|^{2}\right)^{1 / 2}}{\left(1+|y|^{2}\right)^{\gamma}} \mathscr{E}(x-y, t), \\
\rho(x, t):=-\left|\left(1+|x|^{2}\right)^{1 / 2} \nabla v(x, t)-\frac{x}{\left(1+|x|^{2}\right)^{1 / 2}} v(x, t)\right|^{q}, \\
\mu_{\alpha}(x):=\left(1+|x|^{2}\right)^{\alpha} \Delta u_{0}(x), \quad \sigma_{\beta}(x):=\left(1+|x|^{2}\right)^{\beta} \Delta u_{1}(x) .
\end{gathered}
$$

Справедлива следующая лемма.

Лемма 9.1. Пусть $q>1 u v(x, t) \in \mathbb{C}\left([0, T] ; \mathbb{C}_{b}^{1,0}\left(\left(1+|x|^{2}\right)^{1 / 2} ; \mathbb{R}^{3}\right)\right)$. Тогда функиия $\rho(x, t)$ принадлежит классу $\mathbb{C}\left([0, T] ; \mathbb{C}_{b}\left(\mathbb{R}^{3}\right)\right)$ и справедлива следующая оченка:

$$
\begin{gathered}
\sup _{t \in[0, T]}\left|\rho^{1}(x, t)-\rho^{2}(x, t)\right|_{0} \leqslant q \max \left\{\left\|v_{1}\right\|_{0, T}^{q-1},\left\|v_{2}\right\|_{0, T}^{q-1}\right\}\left\|v_{1}-v_{2}\right\|_{0, T}, \\
\rho^{k}(x, t):=\left|\left(1+|x|^{2}\right)^{1 / 2} \nabla v_{k}(x, t)-\frac{x}{\left(1+|x|^{2}\right)^{1 / 2}} v_{k}(x, t)\right|^{q}, \quad k=1,2, \\
\|v\|_{0, T}:=\sup _{t \in[0, T]}|v(x, t)|_{1} .
\end{gathered}
$$

Доказательство. Прежде всего докажем, что в условиях леммы $\rho(x, t) \in \mathbb{C}\left([0, T] ; \mathbb{C}_{b}\left(\mathbb{R}^{3}\right)\right)$. С одной стороны, справедлива цепочка неравенств

$$
\sup _{(x, t) \in \mathbb{R}^{3} \otimes[0, T]}|\rho(x, t)| \leqslant c_{1}(q) \sup _{(x, t) \in \mathbb{R}^{3} \otimes[0, T]}\left[\left(1+|x|^{2}\right)^{q}|\nabla v(x, t)|^{q}+|v(x, t)|^{q}\right]=A(q, T)<+\infty .
$$

Пусть $\left(x_{1}, t_{1}\right),\left(x_{2}, t_{2}\right) \in \mathbb{R}^{3} \otimes[0, T]$. Тогда справедливо поточечное неравенство 


$$
\begin{aligned}
& \left|\rho\left(x_{1}, t_{1}\right)-\rho\left(x_{2}, t_{2}\right)\right| \leqslant \\
\leqslant & q \max \left\{J_{1}^{q-1}, J_{2}^{q-1}\right\}\left[\left|\left(1+\left|x_{1}\right|^{2}\right)^{1 / 2} \nabla v\left(x_{1}, t_{1}\right)-\left(1+\left|x_{2}\right|^{2}\right)^{1 / 2} \nabla v\left(x_{2}, t_{2}\right)\right|+\left|v\left(x_{1}, t_{1}\right)-v\left(x_{2}, t_{2}\right)\right|\right],
\end{aligned}
$$

где

$$
J_{k}:=\left|\left(1+\left|x_{k}\right|^{2}\right)^{1 / 2} \nabla v\left(x_{k}, t_{k}\right)-\frac{x_{k}}{\left(1+\left|x_{k}\right|^{2}\right)^{1 / 2}} v\left(x_{k}, t_{k}\right)\right|, \quad k=1,2 .
$$

Поскольку $v(x, t) \in \mathbb{C}\left([0, T] ; \mathbb{C}_{b}^{1,0}\left(\left(1+|x|^{2}\right)^{1 / 2} ; \mathbb{R}^{3}\right)\right)$, то при $\left|\left(x_{1}, t_{1}\right)-\left(x_{2}, t_{2}\right)\right| \rightarrow+0$ правая часть (9.12) стремится к нулю. В сочетании с (9.11) это приводит к выводу о том, что $\rho(x, t) \in$ $\mathbb{C}_{b}\left(\mathbb{R}^{3} \otimes[0, T]\right)$.

Теперь положим в неравенстве (9.12) $x_{1}=x_{2}=x$ и возьмем supremum по $x \in \mathbb{R}^{3}$. В результате получим следующее неравенство:

$$
\sup _{x \in \mathbb{R}^{3}}\left|\rho\left(x, t_{1}\right)-\rho\left(x, t_{2}\right)\right| \leqslant q A^{(q-1) / q}(q, T) \sup _{x \in \mathbb{R}^{3}}\left|v\left(x, t_{1}\right)-v\left(x, t_{2}\right)\right|_{1} \rightarrow+0
$$

при $\left|t_{1}-t_{2}\right| \rightarrow+0$. Таким образом, $\rho(x, t) \in \mathbb{C}\left([0, T] ; \mathbb{C}_{b}\left(\mathbb{R}^{3}\right)\right)$. Неравенство (9.8) вытекает из оценки вида (9.12).

Перепишем интегральное уравнение (9.4) в следующем операторном виде:

$$
v(x, t)=Q(v)(x, t)=h_{0}(x, t)+H(v)(x, t),
$$

где

$$
\begin{gathered}
H(v)(x, t)=\int_{0}^{t} \int_{\mathbb{R}^{3}} G_{q}(x, y, t-\tau) \rho(y, \tau) d y d \tau, \\
h_{0}(x, t)=\int_{\mathbb{R}^{3}} \frac{\partial G_{\alpha}(x, y, t)}{\partial t} \mu_{\alpha}(y) d y+\int_{\mathbb{R}^{3}} G_{\beta}(x, y, t) \sigma_{\beta}(y) d y .
\end{gathered}
$$

Отметим, что из теорем 8.1, 8.3 и леммы 9.1 при условиях $q>3 / 2, \alpha>3 / 2$ и $\beta>3 / 2$ вытекает, что

$$
\begin{gathered}
H(\cdot): \mathbb{C}\left([0, T] ; \mathbb{C}_{b}^{1,0}\left(\left(1+|x|^{2}\right)^{1 / 2} ; \mathbb{R}^{3}\right)\right) \rightarrow \mathbb{C}\left([0, T] ; \mathbb{C}_{b}^{1,0}\left(\left(1+|x|^{2}\right)^{1 / 2} ; \mathbb{R}^{3}\right)\right), \\
h_{0}(x, t) \in \mathbb{C}\left([0, T] ; \mathbb{C}_{b}^{1,0}\left(\left(1+|x|^{2}\right)^{1 / 2} ; \mathbb{R}^{3}\right)\right)
\end{gathered}
$$

Следовательно,

$$
Q(\cdot): \mathbb{C}\left([0, T] ; \mathbb{C}_{b}^{1,0}\left(\left(1+|x|^{2}\right)^{1 / 2} ; \mathbb{R}^{3}\right)\right) \rightarrow \mathbb{C}\left([0, T] ; \mathbb{C}_{b}^{1,0}\left(\left(1+|x|^{2}\right)^{1 / 2} ; \mathbb{R}^{3}\right)\right) .
$$

Рассмотрим в банаховом пространстве $\mathbb{C}\left([0, T] ; \mathbb{C}_{b}^{1,0}\left(\left(1+|x|^{2}\right)^{1 / 2} ; \mathbb{R}^{3}\right)\right)$ относительно нормы $\|\cdot\|_{0, T}$ замкнутый шар

$$
D_{R, T}:=\left\{v(x, t) \in \mathbb{C}\left([0, T] ; \mathbb{C}_{b}^{1,0}\left(\left(1+|x|^{2}\right)^{1 / 2} ; \mathbb{R}^{3}\right)\right):\|v(x, t)\|_{0, T} \leqslant R\right\}, \quad R>0 .
$$

Пусть $R>0$ настолько велико, что справедливо неравенство

$$
\left\|h_{0}(x, t)\right\|_{0, T} \leqslant \frac{R}{2}
$$

С учетом оценок (3.29) и (3.30) при $k=0$ и оценки (9.8) леммы 9.1 справедлива следующая цепочка неравенств:

$$
\begin{aligned}
& \|H(v)(x, t)\|_{0, T} \leqslant B_{1}(q, T) T \sup _{(x, t) \in \mathbb{R}^{3} \otimes[0, T]}|\rho(x, t)|_{0} \leqslant \\
& \leqslant q B_{1}(q, T) T\|v(x, t)\|_{0, T}^{q} \leqslant q B_{1}(q, T) T R^{q} \leqslant \frac{R}{2}
\end{aligned}
$$

при условии, что $T>0$ настолько мало, что

$$
B_{1}(q, T) T R^{q-1} \leqslant \frac{1}{2}
$$


Таким образом, из (9.22) и (9.23) вытекает, что при достаточно малом $T>0$ оператор

$$
Q(\cdot): D_{R, T} \rightarrow D_{R, T}
$$

Осталось воспользоваться оценкой (9.8) и получить следующую цепочку неравенств:

$$
\begin{gathered}
\left\|Q\left(v_{1}\right)-Q\left(v_{2}\right)\right\|_{0, T} \leqslant q T B_{1}(q, T) \max \left\{\left\|v_{1}\right\|_{0, T}^{q-1},\left\|v_{2}\right\|_{0, T}^{q-1}\right\}\left\|v_{1}-v_{2}\right\|_{0, T} \leqslant \\
\leqslant q T B_{1}(q, T) R^{q-1}\left\|v_{1}-v_{2}\right\|_{0, T} \leqslant \frac{1}{2}\left\|v_{1}-v_{2}\right\|_{0, T}
\end{gathered}
$$

при выполнении условия (9.24) малости $T>0$. В силу принципа сжимающих отображений в шаре $D_{R, T}$ существует единственное решение интегрального уравнения (9.4). Дальше нужно воспользоваться методом продолжения решения интегральных уравнений в классе $\mathbb{C}([0, T] ; \mathbb{B})$, где $\mathbb{B}$ - банахово пространство (см. [12]).

Таким образом, доказана следующая лемма.

Лемма 9.2. Пусть $q>3 / 2 u u_{0}(x) \in \mathbb{C}_{b}^{(2)}\left(\left(1+|x|^{2}\right)^{\alpha} ; \mathbb{R}^{3}\right), u_{1}(x) \in \mathbb{C}_{b}^{(2)}\left(\left(1+|x|^{2}\right)^{\beta} ; \mathbb{R}^{3}\right)$ npu условиях $\alpha>3 / 2, \beta>3 / 2$. Тогда найдется такое $T_{0}=T_{0}\left(u_{0}, u_{1}\right)>0$, что для каждого $T \in\left(0, T_{0}\right)$ существует единственное решение $v(x, t)$ интегрального уравнения $(9.4)$ в классе $\mathbb{C}\left([0, T] ; \mathbb{C}_{b}^{1,0}\left(\left(1+|x|^{2}\right)^{1 / 2} ; \mathbb{R}^{3}\right)\right)$, причем либо $T_{0}=+\infty$, либо $T_{0}<+\infty$, и в этом последнем случае выполнено следующее предельное свойство:

$$
\lim _{T \uparrow T_{0}}\|v\|_{0, T}=+\infty
$$

Из этой леммы вытекает следующее утверждение относительно разрешимости интегрального уравнения (9.1).

Лемма 9.3. Пусть $q>3 / 2 u u_{0}(x) \in \mathbb{C}_{b}^{(2)}\left(\left(1+|x|^{2}\right)^{\alpha} ; \mathbb{R}^{3}\right), u_{1}(x) \in \mathbb{C}_{b}^{(2)}\left(\left(1+|x|^{2}\right)^{\beta} ; \mathbb{R}^{3}\right)$ npu условиях $\alpha>3 / 2, \beta>3 / 2$. Тогда найдется такое $T_{0}=T_{0}\left(u_{0}, u_{1}\right)>0$, что для каждого $T \in\left(0, T_{0}\right)$ существует единственное решение $u(x, t)$ интегрального уравнения $(9.4)$ в классе $\mathbb{C}\left([0, T] ; \mathbb{C}_{b}^{1,1 / 2}\left(\left(1+|x|^{2}\right) ; \mathbb{R}^{3}\right)\right)$, причем либо $T_{0}=+\infty$, либо $T_{0}<+\infty$, и в этом последнем случае выполнено следующее предельное свойство:

$$
\lim _{T \uparrow T_{0}}\left\|\left(1+|x|^{2}\right)^{1 / 2} u(x, t)\right\|_{0, T}=+\infty .
$$

Кроме того, из лемм 9.1 и 9.2 получаем, что при $q>3 / 2$

$$
\rho_{1}(x, t)=-|\nabla u(x, t)|^{q} \in \mathbb{C}\left([0, T] ; \mathbb{C}_{b}\left(\left(1+|x|^{2}\right)^{q} ; \mathbb{R}^{3}\right)\right)
$$

для каждого $T \in\left(0, T_{0}\right)$, где время $T_{0}=T_{0}\left(u_{0}, u_{1}\right)>0$ определено в лемме 9.2. Из теоремы 8.5 вытекает следующее утверждение.

Теорема 9.1. Пусть $q>3 / 2 u$

$$
u_{0}(x) \in \mathbb{C}_{b}^{(2)}\left(\left(1+|x|^{2}\right)^{\alpha} ; \mathbb{R}^{3}\right), \quad u_{1}(x) \in \mathbb{C}_{b}^{(2)}\left(\left(1+|x|^{2}\right)^{\beta} ; \mathbb{R}^{3}\right)
$$

при $\alpha>3 / 2$ и $\beta>3 / 2$. Тогда существует единственное слабое локальное во времени решение $u(x, t)$ задачи Коши (7.1), (7.2) в смысле определения 7.1 в классе $\mathbb{C}\left([0, T] ; \mathbb{C}_{b}^{1,1 / 2}\left(\left(1+|x|^{2}\right) ; \mathbb{R}^{3}\right)\right)$, которое для каждого $T \in\left(0, T_{0}\right)$ принадлежит классу

$$
u(x, t) \in \mathbb{C}^{(2)}\left([0, T] ; \mathbb{C}_{b}^{1,1 / 2}\left(\left(1+|x|^{2}\right) ; \mathbb{R}^{3}\right)\right)
$$

и удовлетворяет начальным условиям

$$
u(x, 0)=u_{0}(x), \quad \frac{\partial u}{\partial t}(x, 0)=u_{1}(x) \quad \text { для всех } x \in \mathbb{R}^{3},
$$

а момент времени $T_{0}=T_{0}\left(u_{0}, u_{1}\right)>0$ определен в лемме 9.3 . 
Доказательство. Шаг 1. Существование. Заметим, что интегральное уравнение (9.4) можно записать в следующем виде:

$$
u(x, t)=L(x, t),
$$

где функция $L(x, t)$ определена равенством (8.113), в котором функция $\rho_{1}(x, t)$ определена равенством (9.29). Выполнены все условия теоремы 8.5. Осталось заметить, что тогда для каждого $t \in[0, T]$ справедливо равенство (8.115). В частности, в качестве пробной функции $\phi(x) \in \mathscr{D}\left(\mathbb{R}^{3}\right)$ можно взять произвольную функцию $\phi(x, t) \in \mathscr{D}\left(\mathbb{R}^{3} \otimes(-\infty, T)\right)$, и мы получим равенство

$$
\left\langle\mathfrak{M}_{x, t}[u](x, t), \phi(x, t)\right\rangle=-\left\langle|\nabla u(x, t)|^{q}, \phi(x, t)\right\rangle \quad \text { для всех } t \in[0, T],
$$

где оператор $\mathfrak{M}_{x, t}$ определен равенством (8.116) и производные по координатам понимаются в смысле обобщенных функций из $\mathscr{D}^{\prime}\left(\mathbb{R}^{3}\right)$. Поскольку, как мы уже доказали, $u(x, t) \in$ $\mathbb{C}^{(2)}\left([0, T] ; \mathbb{C}_{b}^{1,1 / 2}\left(\left(1+|x|^{2}\right) ; \mathbb{R}^{3}\right)\right)$, левую часть равенства $(9.32)$ можно преобразовать к виду

$$
\left\langle\mathfrak{M}_{x, t}[u](x, t), \phi(x, t)\right\rangle=-\int_{\mathbb{R}^{3}}\left(\nabla \frac{\partial^{2} u(x, t)}{\partial t^{2}}, \nabla \phi(x, t)\right) d x-\int_{\mathbb{R}^{3}} \sum_{j=1}^{3} \omega_{j}^{2} u_{x_{j}}(x, t) \phi_{x_{j}}(x, t) d x .
$$

Из равенств (9.32) и (9.33) вытекает равенство

$$
\int_{\mathbb{R}^{3}}\left(\nabla \frac{\partial^{2} u(x, t)}{\partial t^{2}}, \nabla \phi(x, t)\right) d x+\int_{\mathbb{R}^{3}} \sum_{j=1}^{3} \omega_{j}^{2} u_{x_{j}}(x, t) \phi_{x_{j}}(x, t) d x=\int_{\mathbb{R}^{3}}|\nabla u(x, t)|^{q} \phi(x, t) d x,
$$

справедливое для любой пробной функции $\phi(x, t) \in \mathscr{D}\left(\mathbb{R}^{3} \otimes(-\infty, T)\right)$. В рассматриваемом классе гладкости мы можем проинтегрировать обе части равенства (9.34) по $t \in(0, T)$ для каждого $T \in$ $\left(0, T_{0}\right)$; после интегрирования по частям получим равенство (7.7) из определения 7.1 локального во времени слабого решения задачи Коши (7.1), (7.2).

Шаг 2. Единственность. Рассмотрим интегральное уравнение

$$
\begin{aligned}
\widetilde{u}(x, t)=- & \int_{-\infty}^{t} \int_{\mathbb{R}^{3}} \mathscr{E}(x-y, t-\tau)|\nabla \widetilde{u}(y, \tau)|^{q} d y d \tau+ \\
& +\int_{\mathbb{R}^{3}} \frac{\partial \mathscr{E}(x-y, t)}{\partial t} \Delta u_{0}(y) d y+\int_{\mathbb{R}^{3}} \mathscr{E}(x-y, t) \Delta u_{1}(y) d y .
\end{aligned}
$$

В силу леммы 9.3 это интегральное уравнение имеет единственное решение в классе функций $\widetilde{u}(x, t)$, которые обращаются в нуль при $t<0$ и принадлежат классу $\mathbb{C}\left([0, T] ; \mathbb{C}_{b}^{1,1 / 2}\left(\left(1+|x|^{2}\right) ; \mathbb{R}^{3}\right)\right)$ при $t \in[0, T]$. Напомним, что $\mathscr{E}(x, t)=0$ при $t<0$. Осталось воспользоваться теоремой 7.1 , из которой вытекает, что всякое локальное во времени слабое решение $u(x, t)$ задачи Коши $(7.1),(7.2)$ является решением интегрального уравнения (9.35), в котором $\widetilde{u}(x, t)=0$ при $t<0$.

Теперь задача заключается в том, чтобы доказать отсутствие локальных во времени слабых решений задачи Коши (7.1), (7.2) в смысле определения 7.1 при $q \in(1,3 / 2]$. Прежде всего заметим, что имеет место следующее утверждение.

Лемма 9.4. Пусть и $(x, t)$ - слабое локальное во времени решение задачи Коши (7.1), (7.2) в смысле определения 7.1. Тогда равенство (7.7) справедливо для любой функиии $\phi(x, t)=$ $\phi_{1}(x) \phi_{2}(t)$, где $\phi_{1}(x) \in \mathscr{D}\left(\mathbb{R}^{3}\right)$ u $\phi_{2}(t) \in \mathbb{C}_{0}^{(2)}[0, T]$.

Доказательство основано на плотности вложения

$$
\mathscr{D}(-\infty, T) \stackrel{d s}{\complement} \mathbb{C}_{0}^{(2)}[0, T],
$$

которое доказано в приложении, а также на определении пространства $W_{q, \operatorname{loc}}^{1,0}\left(\mathbb{R}^{3} \otimes[0, T]\right)$ и очевидном вложении $\mathscr{D}\left(\mathbb{R}^{3}\right) \otimes \mathscr{D}(-\infty, T) \subset \mathscr{D}\left(\mathbb{R}^{3} \otimes(-\infty, T)\right)$. 
Определение 9.1. Будем говорить, что пара функций $u_{0}(x), u_{1}(x) \in W_{q}^{1}\left(\mathbb{R}^{3}\right)$ принадлежит классу начальных функций $M^{1}$ (обозначение $\left(u_{0}, u_{1}\right) \in M^{1}$ ), если найдется такой шар $O\left(x_{0}, R_{0}\right) \subset$ $\mathbb{R}^{3}$ положительного радиуса, что

$$
\left(\Delta u_{0}(x)\right)^{2}+\left(\Delta u_{1}(x)\right)^{2}>0
$$

на множестве из $O\left(x_{0}, R_{0}\right)$ положительной меры Лебега.

Теорема 9.2. Пусть $1<q \leqslant 3 / 2$. Тогда в классе начальных функций $\left(u_{0}, u_{1}\right) \in M^{1}$ локальное во времени слабое решение задачи Коши (7.1), (7.2) в смысле определения 7.1 отсутствует для любого $T>0$.

Доказательство. Пусть $u(x, t)$ - локальное во времени слабое решение задачи Коши $(7.1),(7.2)$ в смысле определения 7.1 для некоторого $T=T_{1}>0$. С учетом леммы 9.4 в качестве пробной функции $\phi(x, t)=\phi_{1}(x) \phi_{2}(t)$ в равенстве $(7.7)$ возьмем произведение

$$
\phi(x, t)=\phi_{1}(x) \phi_{2}(t),
$$

где

$$
\phi_{1}(x)=\phi_{0}\left(\frac{|x|^{2}}{R^{2}}\right), \quad \phi_{0}(s)= \begin{cases}1, & \text { если } 0 \leqslant s \leqslant 1, \\ 0, & \text { если } s \geqslant 2,\end{cases}
$$

причем функция $\phi_{0}(s) \in \mathbb{C}_{0}^{\infty}[0,+\infty)$ монотонно невозрастающая, а функция $\phi_{2}(t)$ имеет следующий явный вид:

$$
\phi_{2}(t)=\left(1-\frac{t}{T}\right)^{\lambda} \in \mathbb{C}_{0}^{(2)}[0, T] \quad \lambda>2 q^{\prime}, \quad q^{\prime}=\frac{q}{q-1} .
$$

Справедливы следующие оценки:

$$
\begin{gathered}
\left|\int_{0}^{T} \int_{\mathbb{R}^{3}}\left(\nabla u(x, t), \nabla \frac{\partial^{2} \phi(x, t)}{\partial t^{2}}\right) d x d t\right| \leqslant I^{1 / q} c_{1}(R, T), \\
\left|\sum_{j=1}^{3} \omega_{j}^{2} \int_{0}^{T} \int_{\mathbb{R}^{3}} u_{x_{j}}(x, t) \phi_{x_{j}}(x, t) d x d t\right| \leqslant \sum_{j=1}^{3} \omega_{j}^{2} c_{2 j}(R, T) I^{1 / q}, \\
\left|\int_{\mathbb{R}^{3}}\left(\nabla \phi(x, 0), \nabla u_{1}(x)\right) d x\right| \leqslant c_{3}(R)\left\|\left|\nabla u_{1}\right|\right\|_{q}, \\
\left|\int_{\mathbb{R}^{3}}\left(\nabla \frac{\partial \phi}{\partial t}(x, 0), \nabla u_{0}(x)\right) d x\right| \leqslant \frac{\lambda}{T} c_{3}(R)\left\|\left|\nabla u_{0}\right|\right\|_{q},
\end{gathered}
$$

где введены обозначения

$$
\begin{gathered}
c_{1}(R, T):=\left(\int_{0}^{T} \int_{\mathbb{R}^{3}} \frac{\left|\nabla \frac{\partial^{2} \phi(x, t)}{\partial t^{2}}\right|^{q^{\prime}}}{\phi^{q^{\prime} / q}(x, t)} d x d t\right)^{1 / q^{\prime}}=c_{11}(T)\left(\int_{\mathbb{R}^{3}} \frac{\left|\nabla \phi_{1}(x)\right|^{q^{\prime}}}{\phi_{1}^{q^{\prime} / q}(x)} d x\right)^{1 / q^{\prime}}= \\
c_{2 j}(R, T):=\left(\int_{0} \int_{\mathbb{R}^{3}} \frac{\left|\phi_{x_{j}}(x, t)\right|^{q^{\prime}}}{\phi(x, t)^{q^{\prime} / q}} d x d t\right)^{1 / q^{\prime}} \leqslant\left(\int_{0}^{T} \int_{\mathbb{R}^{3}} \frac{|\nabla \phi(x, t)|^{q^{\prime}}}{\phi(x, t)^{q^{\prime} / q}} d x d t\right)^{1 / q^{\prime}}= \\
=c_{20}(T) R^{\left(3-q^{\prime}\right) / q^{\prime}}, \\
=
\end{gathered}
$$




$$
\begin{gathered}
c_{3}(R):=\left(\int_{\mathbb{R}^{3}}\left|\nabla \phi_{1}(x)\right|^{q^{\prime}} d x\right)^{1 / q^{\prime}}=c_{30} R^{\left(3-q^{\prime}\right) / q^{\prime}}, \\
I:=\int_{0}^{T} \int_{\mathbb{R}^{3}} \phi(x, t)|\nabla u(x, t)|^{q} d x d t .
\end{gathered}
$$

Воспользуемся оценками (9.39)-(9.45); с учетом обозначения (9.46) из равенства (7.7) получим неравенство

$$
\begin{aligned}
& R^{\left(3-q^{\prime}\right) / q^{\prime}}\left(c_{11}(T) c_{10}+3 \omega^{2} c_{20}(T)\right) I^{1 / q}+ \\
& +R^{\left(3-q^{\prime}\right) / q^{\prime}}\left(c_{30}\left\|\left|\nabla u_{1}\right|\right\|_{q}+c_{30} \frac{\lambda}{T}\left\|\left|\nabla u_{0}\right|\right\|_{q}\right) \geqslant I, \quad \omega^{2}:=\max \left\{\omega_{1}^{2}, \omega_{2}^{2}, \omega_{3}^{2}\right\} .
\end{aligned}
$$

Используя арифметическое неравенство Гельдера

$$
a^{1 / q^{\prime}} b^{1 / q} \leqslant \frac{a}{q^{\prime}}+\frac{b}{q}, \quad a, b \geqslant 0, \quad \frac{1}{q}+\frac{1}{q^{\prime}}=1,
$$

из неравенства (9.47) получим

$$
R^{3-q^{\prime}}\left(c_{11}(T) c_{10}+3 \omega^{2} c_{20}(T)\right)^{q^{\prime}}+q^{\prime} R^{\left(3-q^{\prime}\right) / q^{\prime}}\left(c_{30}\left\|\left|\nabla u_{1}\right|\right\|_{q}+c_{30} \frac{\lambda}{T}\left\|\left|\nabla u_{0}\right|\right\|_{q}\right) \geqslant I .
$$

Рассмотрим случай, когда $1<q$ и $q^{\prime} \leqslant 3$. В этом случае

$$
1<q \leqslant \frac{3}{2} \text {. }
$$

Теперь нужно отдельно рассмотреть случай $1<q<3 / 2$ и критический случай $q=3 / 2$. В целом рассуждения повторяют соответствующие рассуждения из [10]. В частности, используя классическую теорему Беппо Леви, в случае $1<q<3 / 2$ в пределе при $R \rightarrow+\infty$ из неравенства (9.48) получим равенство

$$
\int_{0}^{T} \int_{\mathbb{R}^{3}}\left(1-\frac{t}{T}\right)^{\lambda}|\nabla u(x, t)|^{q} d x d t=0 .
$$

Это же равенство получается чуть сложнее и в критическом случае $q=3 / 2$. Из (9.50) вытекает, что

$$
u(x, t)=F(t) \quad \text { для почти всех } \quad(x, t) \in \mathbb{R}^{3} \otimes[0, T] .
$$

Из (7.7) и (9.51) получим равенство

$$
\int_{\mathbb{R}^{3}}\left[\left(\nabla \frac{\partial \phi}{\partial t}(x, 0), \nabla u_{0}(x)\right)-\left(\nabla \phi(x, 0), \nabla u_{1}(x)\right)\right] d x=0
$$

для произвольной функции $\phi(x, t)=\phi_{1}(x) \phi_{2}(t)$, где $\phi_{2}(t)$ определена равенством $(9.38)$, a $\phi_{1}(x) \in$ $\mathscr{D}\left(\mathbb{R}^{3}\right)$. Поскольку $\left(u_{0}, u_{1}\right) \in M^{1}$, то из равенства $(9.52)$ получим

$$
\int_{\mathbb{R}^{3}}\left[\frac{\lambda}{T} \Delta u_{0}(x)+\Delta u_{1}(x)\right] \phi_{1}(x) d x=0
$$

для любой функции $\phi_{1}(x) \in \mathscr{D}\left(\mathbb{R}^{3}\right)$ с носителем supp $\phi_{1}(x) \subset O\left(x_{0}, R_{0}\right)$. В силу основной леммы вариационного исчисления приходим к равенству

$$
\frac{\lambda}{T} \Delta u_{0}(x)+\Delta u_{1}(x)=0 \quad \text { для почти всех } x \in O\left(x_{0}, R_{0}\right) .
$$


Отметим, что если $u(x, t)$ - слабое решение задачи Коши для некоторого $T=T_{1}>0$, то оно же является слабым решением и для любого $T \in\left(0, T_{1}\right]$, но тогда равенство (9.54) должно быть выполнено и для всех $T \in\left(0, T_{1}\right]$. Следовательно,

$$
\Delta u_{0}(x)=0, \quad \Delta u_{1}(x)=0 \quad \text { для почти всех } x \in O\left(x_{0}, R_{0}\right),
$$

что противоречит определению класса функций $M^{1}$.

Теперь наша задача доказать, что в некотором классе $N^{1}$ начальных функций $u_{0}(x), u_{1}(x)$ при $q>3 / 2$ локальное во времени слабое решение задачи Коши (7.1), (7.2) в смысле определения 7.1 разрушается за конечное время $T_{0}=T_{0}\left(u_{0}, u_{1}\right)>0$, определенное в лемме 9.3 .

Определение 9.2. Будем говорить, что начальные функции $u_{0}, u_{1}$ принадлежат классу $N^{1}$ (обозначение $\left(u_{0}, u_{1}\right) \in N^{1}$ ), если найдется такая неотрицательная пробная функция $\phi(x, t) \in$ $\mathscr{D}\left(\mathbb{R}^{3} \otimes(-\infty,+\infty)\right)$, что конечна нелинейная емкость

$$
E_{1}(\phi):=\int_{0}^{+\infty} \int_{\mathbb{R}^{3}} \frac{\left|\nabla \frac{\partial^{2} \phi(x, t)}{\partial t^{2}}\right|^{q^{\prime}}}{\phi^{q^{\prime} / q}(x, t)} d x d t+\sum_{j=1}^{3} \omega_{j}^{2} \int_{0}^{+\infty} \int_{\mathbb{R}^{3}} \frac{\left|\phi_{x_{j}}(x, t)\right|^{q^{\prime}}}{\phi^{q^{\prime} / q}(x, t)} d x d t<+\infty
$$

и при этом выполнено неравенство

$$
\frac{1}{q^{\prime}}\left(\frac{2}{q}\right)^{q^{\prime} / q} E_{1}(\phi)+\int_{\mathbb{R}^{3}}\left[\left(\nabla \frac{\partial \phi}{\partial t}(x, 0), \nabla u_{0}(x)\right)-\left(\nabla \phi(x, 0), \nabla u_{1}(x)\right)\right] d x<0 .
$$

Замечание 9.1. Заметим, что в [14] доказано существование такой пробной функции, что нелинейная емкость (9.56) конечна. Фиксируем эту пробную функцию $\phi(x, t)$. Пусть выполнено неравенство

$$
\int_{\mathbb{R}^{3}}\left[\left(\nabla \frac{\partial \phi}{\partial t}(x, 0), \nabla u_{0}(x)\right)-\left(\nabla \phi(x, 0), \nabla u_{1}(x)\right)\right] d x<0 .
$$

Если вместо начальных функций $u_{0}(x)$ и $u_{1}(x)$ рассмотреть функции $r u_{0}(x)$ и $r u_{1}(x)$ при $r>0$, то при достаточно большом $r>0$ будет выполнено неравенство (9.57). Пусть теперь выполнено неравенство

$$
\int_{\mathbb{R}^{3}}\left[\left(\nabla \frac{\partial \phi}{\partial t}(x, 0), \nabla u_{0}(x)\right)-\left(\nabla \phi(x, 0), \nabla u_{1}(x)\right)\right] d x>0 .
$$

Если вместо начальных функций $u_{0}(x)$ и $u_{1}(x)$ рассмотреть функции $r u_{0}(x)$ и $r u_{1}(x)$ при $r<0$, то при достаточно большом $r<0$ будет выполнено неравенство (9.57). Таким образом, определение 9.2 корректно.

Справедлива следующая теорема.

Теорема 9.3. Пусть $q>3 / 2 u\left(u_{0}, u_{1}\right) \in N^{1}$. Тогда существует единственное локальное во времени слабое решение задачи Коши (7.1), (7.2) в смысле определения 7.1 в классе $\mathbb{C}\left([0, T] ; \mathbb{C}_{b}^{1,1 / 2}\left(\left(1+|x|^{2}\right) ; \mathbb{R}^{3}\right)\right)$ для всех $T \in\left(0, T_{0}\right)$ класса гладкости $\mathbb{C}^{(2)}\left([0, T] ; \mathbb{C}_{b}^{1,1 / 2}\left(\left(1+|x|^{2}\right) ; \mathbb{R}^{3}\right)\right)$, причем $0<T_{0}<+\infty$ и слабое решение разрушается в момент времени $T_{0}$ в следующем смысле:

$$
\lim _{T \uparrow T_{0}}\left\|\left(1+|x|^{2}\right)^{1 / 2} u(x, t)\right\|_{0, T}=+\infty .
$$

Доказательство. Предположим, что $T_{0}=+\infty$, где $T_{0}$ определено в лемме 9.3 . Тогда точно так же, как при доказательстве теоремы 9.1 , можно убедиться, что для любого $T>0$ существует единственное в классе $\mathbb{C}\left([0, T] ; \mathbb{C}_{b}^{1,1 / 2}\left(\left(1+|x|^{2}\right) ; \mathbb{R}^{3}\right)\right)$ слабое глобальное во времени решение $u(x, t)$ задачи Коши $(7.1),(7.2)$ в смысле определения 7.2 класса гладкости $\mathbb{C}^{(2)}\left([0, T] ; \mathbb{C}_{b}^{1,1 / 2}\left(\left(1+|x|^{2}\right) ; \mathbb{R}^{3}\right)\right)$ 
для любого $T>0$. Докажем, что для начальных функций $\left(u_{0}, u_{1}\right) \in N^{1}$ мы получим противоречивое неравенство. Действительно, используя неравенство Гельдера и трехпараметрическое неравенство Юнга

$$
a b \leqslant \varepsilon a^{q}+\frac{1}{q^{\prime}(\varepsilon q)^{q^{\prime} / q}}, \quad a, b \geqslant 0, \quad \varepsilon>0, \quad \frac{1}{q}+\frac{1}{q^{\prime}}=1,
$$

из равенства (7.8) можно получить (стандартной техникой работы [14]) следующую оценку:

$$
\begin{aligned}
\frac{1}{q^{\prime}} \frac{1}{(\varepsilon q)^{q^{\prime} / q}} E_{1}(\phi)+\int_{\mathbb{R}^{3}}\left[\left(\nabla \frac{\partial \phi}{\partial t}(x, 0), \nabla u_{0}(x)\right)-\left(\nabla \phi(x, 0), \nabla u_{1}(x)\right)\right] d x \geqslant & \\
& \geqslant(1-2 \varepsilon) \int_{0}^{+\infty} \int_{\mathbb{R}^{3}} \phi(x, t)|\nabla u(x, t)|^{q} d x d t
\end{aligned}
$$

для любой функции $\phi(x, t) \in \mathscr{D}\left(\mathbb{R}^{3} \otimes(-\infty,+\infty)\right)$. Положим $\varepsilon=1 / 2$ в неравенстве (9.62). Тогда получим

$$
\frac{1}{q^{\prime}}\left(\frac{2}{q}\right)^{q^{\prime} / q} E_{1}(\phi)+\int_{\mathbb{R}^{3}}\left[\left(\nabla \frac{\partial \phi}{\partial t}(x, 0), \nabla u_{0}(x)\right)-\left(\nabla \phi(x, 0), \nabla u_{1}(x)\right)\right] d x \geqslant 0
$$

для любой неотрицательной функции $\phi(x, t) \in \mathscr{D}\left(\mathbb{R}^{3} \otimes(-\infty,+\infty)\right)$. Поскольку $\left(u_{0}, u_{1}\right) \in N^{1}$, то найдется такая неотрицательная пробная функция $\phi(x, t)$, что будет выполнено неравенство (9.57), которое входит в противоречие с неравенством (9.63). Таким образом, доказано, что время $T_{0}$ из леммы 9.3 конечно. Но тогда выполнено неравенство (9.28). Разрушение локального во времени слабого решения задачи Коши (7.1), (7.2) в смысле определения 7.1 доказано.

10. ЗАДАЧА КОШИ (7.3), (7.4): СУЩЕСТВОВАНИЕ ЛОКАЛЬНЫХ И ОТСУТСТВИЕ ЛОКАЛЬНЫХ И ГЛОБАЛЬНЫХ ВО ВРЕМЕНИ СЛАБЫХ РЕШЕНИЙ

Рассмотрим следующее вспомогательное интегральное уравнение:

$$
\begin{aligned}
& u(x, t)=\int_{0}^{t} \int_{\mathbb{R}^{3}} \mathscr{E}(x-y, t-\tau) \frac{\partial|\nabla u(y, \tau)|^{q}}{\partial \tau} d y d \tau+ \\
& \quad+\int_{\mathbb{R}^{3}} \frac{\partial \mathscr{E}(x-y, t)}{\partial t} \Delta u_{0}(y) d y+\int_{\mathbb{R}^{3}} \mathscr{E}(x-y, t) \Delta u_{1}(y) d y .
\end{aligned}
$$

Перейдя в уравнении (10.1) к новой функции (9.2), получим следующее интегральное уравнение:

$$
\begin{aligned}
v(x, t)=\int_{0} \int_{\mathbb{R}^{3}} G_{q}(x, y, t-\tau) \rho(y, \tau) d y & d \tau+\int_{\mathbb{R}^{3}} \frac{\partial G_{\alpha}(x, y, t)}{\partial t} \mu_{\alpha}(y) d y+ \\
& +\int_{\mathbb{R}^{3}} G_{\beta}(x, y, t) \sigma_{\beta}(y) d y:=U(x, t)+W(x, t)+V(x, t),
\end{aligned}
$$

где

$$
\begin{gathered}
G_{\gamma}(x, y, t):=\frac{\left(1+|x|^{2}\right)^{1 / 2}}{\left(1+|y|^{2}\right)^{\gamma}} \mathscr{E}(x-y, t), \\
\rho(x, t):=\frac{\partial}{\partial t}\left|\left(1+|x|^{2}\right)^{1 / 2} \nabla v(x, t)-\frac{x}{\left(1+|x|^{2}\right)^{1 / 2}} v(x, t)\right|^{q}, \\
\mu_{\alpha}(x):=\left(1+|x|^{2}\right)^{\alpha} \Delta u_{0}(x), \quad \sigma_{\beta}(x):=\left(1+|x|^{2}\right)^{\beta} \Delta u_{1}(x) .
\end{gathered}
$$


Лемма 10.1. Если $q \geqslant 2$ u $v(x, t) \in \mathbb{C}^{(1)}\left([0, T] ; \mathbb{C}_{b}^{1,0}\left(\left(1+|x|^{2}\right)^{1 / 2} ; \mathbb{R}^{3}\right)\right)$, то функция $\rho(x, t)$ принадлежит классу $\mathbb{C}\left([0, T] ; \mathbb{C}_{b}\left(\mathbb{R}^{3}\right)\right)$, причем для любых $v_{k}(x, t) \in \mathbb{C}^{(1)}\left([0, T] ; \mathbb{C}_{b}^{1,0}\left(\left(1+|x|^{2}\right)^{1 / 2} ; \mathbb{R}^{3}\right)\right)$ при $k=1,2$ справедливо следующее неравенство:

$$
\begin{gathered}
\sup _{t \in[0, T]}\left|\rho_{1}(x, t)-\rho_{2}(x, t)\right|_{0} \leqslant d(q) \max \left\{\left\|v_{1}\right\|_{1, T}^{q-1},\left\|v_{2}\right\|_{1, T}^{q-1}\right\}\left\|v_{1}-v_{2}\right\|_{1, T}, \\
\rho_{k}(x, t):=\frac{\partial}{\partial t}\left|\left(1+|x|^{2}\right)^{1 / 2} \nabla v_{k}(x, t)-\frac{x}{\left(1+|x|^{2}\right)^{1 / 2}} v_{k}(x, t)\right|^{q}, \quad k=1,2,
\end{gathered}
$$

əde

$$
\|v\|_{1, T}:=\|v\|_{0, T}+\left\|\frac{\partial v(x, t)}{\partial t}\right\|_{0, T}, \quad\|w\|_{0, T}:=\sup _{t \in[0, T]}|w(x, t)|_{1} .
$$

Доказательство. Пусть

$$
\rho(x, t)=\frac{\partial}{\partial t}|z(x, t)|^{q}, \quad z=z(x, t)=\left(z_{1}(x, t), z_{2}(z, t), z_{3}(x, t)\right), \quad q \geqslant 2 .
$$

Справедливы следующие равенства:

$$
\begin{gathered}
\frac{\partial}{\partial t}|z(x, t)|^{q}=q\left(z_{t}(x, t),|z(z, t)|^{q-2} z(x, t)\right), \\
\rho_{k}(x, t)=\frac{\partial}{\partial t}\left|z_{k}(x, t)\right|^{q}, \quad k=1,2, \\
\rho_{1}-\rho_{2}=q\left(z_{1 t},\left|z_{1}\right|^{q-2} z_{1}\right)-q\left(z_{2 t},\left|z_{2}\right|^{q-2} z_{2}\right)= \\
=q\left(z_{1 t}-z_{2 t},\left|z_{1}\right|^{q-2} z_{1}\right)+q\left(z_{2 t},\left|z_{1}\right|^{q-2} z_{1}-\left|z_{2}\right|^{q-2} z_{2}\right), \\
\left|z_{1}\right|^{q-2} z_{1}-\left|z_{2}\right|^{q-2} z_{2}=\int_{0}^{1} \frac{\partial}{\partial s}\left[\left|s z_{1}+(1-s) z_{2}\right|^{q-2}\left(s z_{1}+(1-s) z_{2}\right)\right] d s= \\
=\int_{0}^{1}\left|s z_{1}+(1-s) z_{2}\right|^{q-2}\left(z_{1}-z_{2}\right) d s+ \\
+(q-2) \int_{0}^{1}\left|s z_{1}+(1-s) z_{2}\right|^{q-4}\left(s z_{1}+(1-s) z_{2}, z_{2}-z_{2}\right)\left(s z_{1}+(1-s) z_{2}\right) d s,
\end{gathered}
$$

из которого вытекает следующая оценка:

$$
\begin{aligned}
\left.|| z_{1}\right|^{q-2} z_{1}-\left|z_{2}\right|^{q-2} z_{2}\left|\leqslant(q-1) \int_{0}^{1}\right| s z_{1}+\left.(1-s) z_{2}\right|^{q-2}\left|z_{1}-z_{2}\right| d s & \leqslant \\
& \leqslant(q-1) \max \left\{\left|z_{1}\right|^{q-2},\left|z_{2}\right|^{q-2}\right\}\left|z_{1}-z_{2}\right| .
\end{aligned}
$$

Из оценки (10.14) и равенства (10.12) вытекает искомая оценка

$$
\left|\rho_{1}-\rho_{2}\right| \leqslant q\left|z_{1}\right|^{q-1}\left|z_{1 t}-z_{2 t}\right|+q(q-1)\left|z_{2 t}\right| \max \left\{\left|z_{1}\right|^{q-2},\left|z_{2}\right|^{q-2}\right\}\left|z_{1}-z_{2}\right|
$$

при $q \geqslant 2$. Сначала положим

$$
z_{k}=\left(1+\left|x^{k}\right|^{2}\right)^{1 / 2} \nabla v\left(x^{k}, t^{k}\right)-\frac{x^{k}}{\left(1+\left|x^{k}\right|^{2}\right)^{1 / 2}} v\left(x^{k}, t^{k}\right), \quad k=1,2,
$$

и из неравенства (10.15) получим, что в условиях леммы $\rho(x, t) \in \mathbb{C}\left([0, T] ; \mathbb{C}_{b}\left(\mathbb{R}^{3}\right)\right)$. Теперь в неравенстве (10.15) положим

$$
z_{k}=\left(1+|x|^{2}\right)^{1 / 2} \nabla v_{k}(x, t)-\frac{x}{\left(1+|x|^{2}\right)^{1 / 2}} v_{k}(x, t), \quad k=1,2,
$$


и получим неравенство (10.6).

Относительно разрешимости интегрального уравнения (10.2) справедливо следующее утверждение.

Лемма 10.2. Пусть $q \geqslant 2, u_{0}(x) \in \mathbb{C}_{b}^{(2)}\left(\left(1+|x|^{2}\right)^{\alpha} ; \mathbb{R}^{3}\right), u_{1}(x) \in \mathbb{C}_{b}^{(2)}\left(\left(1+|x|^{2}\right)^{\beta} ; \mathbb{R}^{3}\right)$ npu условиях $\alpha>3 / 2, \beta>3 / 2$. Тогда найдется такое $T_{0}=T_{0}\left(u_{0}, u_{1}\right)>0$, что для каждого $T \in\left(0, T_{0}\right)$ существует единственное решение $v(x, t)$ интегрального уравнения $(10.2)$ в классе $\mathbb{C}^{(1)}\left([0, T] ; \mathbb{C}_{b}^{1,0}\left(\left(1+|x|^{2}\right)^{1 / 2} ; \mathbb{R}^{3}\right)\right)$, причем либо $T_{0}=+\infty$, либо $T_{0}<+\infty$, и в этом последнем случае выполнено следующее предельное свойство:

$$
\lim _{T \uparrow T_{0}}\|v\|_{1, T}=+\infty
$$

Доказательство этой леммы в целом повторяет доказательство леммы 9.1. Заметим, только, что в рассматриваемом классе гладкости функций $v(x, t) \in \mathbb{C}^{(1)}\left([0, T] ; \mathbb{C}_{b}^{1,0}\left(\left(1+|x|^{2}\right)^{1 / 2} ; \mathbb{R}^{3}\right)\right)$ справедливы поточечные равенства

$$
\begin{aligned}
& \frac{\partial U(x, t)}{\partial t}=\int_{0}^{t} \int_{\mathbb{R}^{3}} \frac{\partial G_{q}(x, y, t-\tau)}{\partial t} \rho(y, \tau) d y, d \tau, \\
& \frac{\partial W(x, t)}{\partial t}=\int_{0}^{t} \int_{\mathbb{R}^{3}} \frac{\partial^{2} G_{q}(x, y, t-\tau)}{\partial t^{2}} \mu_{\alpha}(y) d y, d \tau, \\
& \frac{\partial V(x, t)}{\partial t}=\int_{0}^{t} \int_{\mathbb{R}^{3}} \frac{\partial G_{q}(x, y, t-\tau)}{\partial t} \sigma_{\alpha}(y) d y, d \tau .
\end{aligned}
$$

Осталось воспользоваться методом сжимающих отображений с учетом теоремы 8.2, леммы 10.1 и техникой работы [12] для продолжения решения решения интегрального уравнения (10.2) во времени.

Замечание 10.1. В [12] рассматривалось абстрактное интегральное уравнение вида

$$
u(t)=f_{0}(t)+\int_{0}^{t} K(t, \tau) A(\tau, u(\tau)) d \tau,
$$

для которого применялся алгоритм продолжения решений во времени в банаховом пространстве $\mathbb{C}([0, T] ; \mathbb{B})$. Для наших же целей нужно рассмотреть алгоритм продолжения решения интегрального уравнения в банаховом пространстве $\mathbb{C}^{(1)}([0, T] ; \mathbb{B})$. Он заключается в следующем. Пусть мы доказали принципом сжимающих отображений существование единственного решения $u(t) \in \mathbb{C}^{(1)}\left(\left[0, T^{*}\right] ; \mathbb{B}\right)$ при некотором $T=T^{*}>0$. Тогда уравнение $(10.22)$ при $t>T^{*}$ можно переписать в виде

$$
u(t)=u\left(T^{*}\right)+f^{*}(t)+\int_{T^{*}}^{t} K(t, \tau) A(\tau, u(\tau)) d \tau,
$$

где функция $f^{*}(t)$ известна:

$$
f^{*}(t)=f_{0}(t)-f_{0}\left(T^{*}\right)+\int_{0}^{T^{*}}\left[K(t, \tau)-K\left(T^{*}, \tau\right)\right] A(\tau, u(\tau)) d \tau .
$$

Из (10.22) и из (10.23) с учетом явного вида (10.24) функции $f^{*}(t)$ можно заметить, что

$$
u\left(T^{*}-0\right)=u\left(T^{*}+0\right), \quad \frac{d u}{d t}\left(T^{*}-0\right)=\frac{d u}{d t}\left(T^{*}+0\right) .
$$


Осталось воспользоваться снова методом сжимающих отображений для интегрального уравнения (10.23) и с учетом гладкого сшивания (10.25) доказать существование решения в классе $u(t) \in \mathbb{C}^{(1)}\left(\left[0, T^{* *}\right] ; \mathbb{B}\right)$ при $T^{* *}>T^{*}$. Дальнейшие рассуждения приводят нас к существованию такого максимального $T_{0}=T_{0}\left(u_{0}\right)>0$, для которого выполнена альтернатива в формулировке теоремы.

Из леммы 10.2 вытекает следующее утверждение относительно разрешимости интегрального уравнения (10.1).

Лемма 10.3. Пусть $q \geqslant 2 u u_{0}(x) \in \mathbb{C}_{b}^{(2)}\left(\left(1+|x|^{2}\right)^{\alpha} ; \mathbb{R}^{3}\right), u_{1}(x) \in \mathbb{C}_{b}^{(2)}\left(\left(1+|x|^{2}\right)^{\beta} ; \mathbb{R}^{3}\right)$ npu условиях $\alpha>3 / 2, \beta>3 / 2$. Тогда найдется такое $T_{0}=T_{0}\left(u_{0}, u_{1}\right)>0$, что для каждого $T \in\left(0, T_{0}\right)$ существует единственное решение $u(x, t)$ интегрального уравнения $(10.1)$ в классе $\mathbb{C}^{(1)}\left([0, T] ; \mathbb{C}_{b}^{1,1 / 2}\left(\left(1+|x|^{2}\right) ; \mathbb{R}^{3}\right)\right)$, причем либо $T_{0}=+\infty$, либо $T_{0}<+\infty$, и в этом последнем случае выполнено следующее предельное свойство:

$$
\lim _{T \uparrow T_{0}}\left\|\left(1+|x|^{2}\right)^{1 / 2} u(x, t)\right\|_{1, T}=+\infty .
$$

Из лемм 10.1 и 10.2 вытекает, что

$$
\begin{gathered}
|\nabla u(x, t)|^{q} \in \mathbb{C}^{(1)}\left([0, T] ; \mathbb{C}_{b}\left(\left(1+|x|^{2}\right)^{q} ; \mathbb{R}^{3}\right)\right) \subset \mathbb{C}_{x, t}^{(0,1)}\left(\mathbb{R}^{3} \otimes[0, T]\right), \\
\rho_{1}(x, t)=\frac{\partial}{\partial t}|\nabla u(x, t)|^{q} \in \mathbb{C}\left([0, T] ; \mathbb{C}_{b}\left(\left(1+|x|^{2}\right)^{q} ; \mathbb{R}^{3}\right)\right) .
\end{gathered}
$$

Теорема 10.1. Пусть $q \geqslant 2, u_{0}(x) \in \mathbb{C}_{b}^{(2)}\left(\left(1+|x|^{2}\right)^{\alpha} ; \mathbb{R}^{3}\right), u_{1}(x) \in \mathbb{C}_{b}^{(2)}\left(\left(1+|x|^{2}\right)^{\beta} ; \mathbb{R}^{3}\right)$ npu $\alpha>3 / 2$ и $\beta>3 / 2$. Тогда существует единственное слабое локальное во времени решение и $(x, t)$ задачи Коши (7.3), (7.4) в смьсле определения 7.3 в классе $\mathbb{C}^{(1)}\left([0, T] ; \mathbb{C}_{b}^{1,1 / 2}\left(\left(1+|x|^{2}\right) ; \mathbb{R}^{3}\right)\right)$, которое для каждого $T \in\left(0, T_{0}\right)$ принадлежст классу $u(x, t) \in \mathbb{C}^{(2)}\left([0, T] ; \mathbb{C}_{b}^{1,1 / 2}\left(\left(1+|x|^{2}\right) ; \mathbb{R}^{3}\right)\right) u$ удовлетворяет начальным условиям

$$
u(x, 0)=u_{0}(x), \quad \frac{\partial u}{\partial t}(x, 0)=u_{1}(x) \quad \text { для всех } x \in \mathbb{R}^{3},
$$

а момент времени $T_{0}=T_{0}\left(u_{0}, u_{1}\right)>0$ определен в лемме 10.3 .

Доказательство. Шаг 1. Существование локального решения слабого решения $u(x, t)$ задачи Коши (7.3), (7.4) в смысле определения 7.3 вытекает из (10.28), вида интегрального уравнения (10.1) и теоремы 8.5. Кроме того, нужно провести такие же рассуждения, как на шаге 1 доказательства теоремы 9.1 .

Шаг 2. Единственность слабого решения в классе $\mathbb{C}^{(1)}\left([0, T] ; \mathbb{C}_{b}^{1,1 / 2}\left(\left(1+|x|^{2}\right) ; \mathbb{R}^{3}\right)\right)$ вытекает из доказанного свойства (10.27), определения слабого решения 7.3, теоремы 7.2 и аналогично шагу 2 доказательства теоремы 9.1.

Лемма 10.4. Пусть $и(x, t)$ - слабое локальное во времени решение задачи Коши (7.3), (7.4) в смысле определения 7.3. Тогда равенство (7.9) справедливо для любой функции $\phi(x, t)=$ $\phi_{1}(x) \phi_{2}(t)$, где $\phi_{1}(x) \in \mathscr{D}\left(\mathbb{R}^{3}\right) u \phi_{2}(t) \in \mathbb{C}_{0}^{(2)}[0, T]$

Доказательство в точности повторяет доказательство леммы 9.4.

Определение 10.1. Будем говорить, что функции $u_{0}$ и $u_{1}$ принадлежат классу начальных функций $M^{2}$ (обозначение $\left(u_{0}, u_{1}\right) \in M^{2}$ ), если $u_{0}(x), u_{1}(x) \in W_{q}^{1}\left(\mathbb{R}^{3}\right),\left|\nabla u_{0}(x)\right|=0$ для почти всех $x \in \mathbb{R}^{3}$ и найдется такой шар $O\left(x_{0}, R_{0}\right) \subset \mathbb{R}^{3}$ положительного радиуса, что $\left(\Delta u_{1}(x)\right)^{2}>0$ на множестве из $O\left(x_{0}, R_{0}\right)$ положительной меры Лебега.

Теорема 10.2. Пусть $1<q \leqslant 3 / 2 u\left(u_{0}, u_{1}\right) \in M^{2}$. Тогда локальное во времени слабое решение задачи Коши (7.3), (7.4) в смысле определения 7.3 отсутствует для любого $T>0$. 
Доказательство. Шаг 1. Вывод априорной оценки. Пусть $u(x, t)$-слабое решение задачи Коши (7.3), (7.4) в смысле определения 7.3 при некотором $T=T_{1}>0$. В равенстве (7.9) в качестве пробной функции $\phi(x, t)$ возьмем функцию, определенную равенствами (9.36)-(9.38), где $\lambda>q^{\prime}$. Справедливы следующие оценки:

$$
\begin{gathered}
\left|\int_{0} \int_{\mathbb{R}^{3}}\left(\nabla u(x, t), \nabla \frac{\partial^{2} \phi(x, t)}{\partial t^{2}}\right) d x d t\right| \leqslant b_{1}(R, T) I^{1 / q}, \\
\left|\sum_{j=1}^{3} \int_{0}^{T} \int_{\mathbb{R}^{3}} \omega_{j}^{2} u_{x_{j}}(x, t) \phi_{x_{j}}(x, t) d x d t\right| \leqslant \sum_{j=1}^{3} \omega_{j}^{2} b_{2 j}(R, T) I^{1 / q}, \\
\int_{\mathbb{R}^{3}}\left(\nabla \frac{\partial \phi}{\partial t}(x, 0), \nabla u_{0}(x)\right) d x\left|\leqslant \frac{\lambda}{T} b_{3}(R)\left\|\left|\nabla u_{0}\right|\right\|_{q}=\frac{\lambda}{T} b_{30} R^{\left(3-q^{\prime}\right) / q^{\prime}}\left\|\left|\nabla u_{0}\right|\right\|_{q},\right. \\
\int_{\mathbb{R}^{3}}\left(\nabla \phi(x, 0), \nabla u_{1}(x)\right) d x\left|\leqslant b_{3}(R)\right||| \nabla u_{1}\left|\left\|_{q}=b_{30} R^{\left(3-q^{\prime}\right) / q^{\prime}}\right\|\right| \nabla u_{1} \mid \|_{q}, \\
b_{1}(R, T):=\left(\int_{0}^{T} \int_{\mathbb{R}^{3}} \frac{\left|\nabla \frac{\partial^{2} \phi(x, t)}{\partial t^{2}}\right|^{q^{\prime}}}{\left(-\frac{\partial \phi(x, t)}{\partial t}\right)^{q^{\prime} / q}} d x d t\right)^{1 / q^{\prime}}=b_{10}(T) R^{\left(3-q^{\prime}\right) / q^{\prime}}, \\
b_{2 j}(R, T):=\left(\int_{0}^{T} \int_{\mathbb{R}^{3}} \frac{\left|\phi_{x_{j}}(x, t)\right|^{q^{\prime}}}{\left(-\frac{\partial \phi(x, t)}{\partial t}\right)^{q^{\prime} / q}} d x d t\right)^{T / q^{\prime}}=b_{20 j}(T) R^{\left(3-q^{\prime}\right) / q^{\prime}}, \\
\int_{\mathbb{R}^{3}} \int^{T}\left(-\frac{\partial \phi(x, t)}{\partial t}\right)|\nabla u(x, t)|^{q} d x d t .
\end{gathered}
$$

Теперь рассмотрим случай, когда $1<q$ и $q^{\prime} \geqslant 3$. Это равносильно неравенствам $1<q \leqslant 3 / 2$. Из равенства (7.9) с учетом оценок (10.30)-(10.35) получим следующую оценку:

$$
\begin{aligned}
b_{10}(T) R^{\left(3-q^{\prime}\right) / q^{\prime}} I^{1 / q} & +\sum_{j=1}^{3} \omega_{j}^{2} b_{20 j}(T) R^{\left(3-q^{\prime}\right) / q^{\prime}} I^{1 / q}+ \\
& +\left(\frac{\lambda}{T} b_{30}\left\|\left|\nabla u_{0}\right|\right\|_{q}+\left\|\left|\nabla u_{1}\right|\right\|_{q}\right) R^{\left(3-q^{\prime}\right) / q^{\prime}}+\int_{\mathbb{R}^{3}} \phi_{1}(x)\left|\nabla u_{0}(x)\right|^{q} d x \geqslant I .
\end{aligned}
$$

Теперь воспользуемся трехпараметрическим неравенством Юнга (9.61) при $\varepsilon \in(0,1 / 2)$ и получим из (10.37) следующее неравенство:

$$
\begin{array}{r}
R^{3-q^{\prime}} c_{1}(\varepsilon)\left(b_{10}^{q^{\prime}}(T)+\left(\sum_{j=1}^{3} \omega_{j}^{2} b_{20 j}(T)\right)^{q^{\prime}}\right)+\left(\frac{\lambda}{T} b_{30}\left\|\left|\nabla u_{0}\right|\right\|_{q}+\left\|\left|\nabla u_{1}\right|\right\|_{q}\right) R^{\left(3-q^{\prime}\right) / q^{\prime}}+ \\
+\int_{\mathbb{R}^{3}} \phi_{1}(x)\left|\nabla u_{0}(x)\right|^{q} d x \geqslant(1-2 \varepsilon) I, \quad c_{1}(\varepsilon):=\frac{1}{q^{\prime}} \frac{1}{(q \varepsilon)^{q^{\prime} / q}} .
\end{array}
$$

Отдельно необходимо (как и ранее) рассмотреть случай $1<q<3 / 2$ и критический случай $q=3 / 2$. Например, в случае $1<q<3 / 2$ можно воспользоваться теоремой Беппо Леви и в итоге 
в пределе при $R \rightarrow+\infty$ из неравенства (10.38) получить следующую априорную оценку:

$$
\int_{\mathbb{R}^{3}}\left|\nabla u_{0}(x)\right|^{q} d x \geqslant \frac{\lambda}{T}(1-2 \varepsilon) \int_{0}^{T} \int_{\mathbb{R}^{3}}\left(1-\frac{t}{T}\right)^{\lambda-1}|\nabla u(x, t)|^{q} d x d t, \quad \varepsilon \in(0,1 / 2) .
$$

В пределе при $\varepsilon \rightarrow+0$ из (10.39) вытекает искомая априорная оценка:

$$
\int_{\mathbb{R}^{3}}\left|\nabla u_{0}(x)\right|^{q} d x \geqslant \frac{\lambda}{T} \int_{0}^{T} \int_{\mathbb{R}^{3}}\left(1-\frac{t}{T}\right)^{\lambda-1}|\nabla u(x, t)|^{q} d x d t, \quad \lambda>q^{\prime} .
$$

Шаг 2. Отсутствие локальных решений. Поскольку $\left(u_{0}, u_{1}\right) \in M^{2}$, то из (10.40) следует, что

$$
u(x, t)=F(t) \quad \text { для почти всех } \quad(x, t) \in \mathbb{R}^{3} \otimes[0, T] .
$$

После подстановки этого равенства в (7.9) получим равенство

$$
\int_{\mathbb{R}^{3}}\left[\left(\nabla \frac{\partial \phi}{\partial t}(x, 0), \nabla u_{0}(x)\right)-\left(\nabla \phi(x, 0), \nabla u_{1}(x)\right)\right] d x=0,
$$

справедливое для всех $T \in\left(0, T_{1}\right]$ и для всех функций $\phi(x, t)=\phi_{1}(x) \phi_{2}(t)$, определенных равенствами (9.36)-(9.38). Дальнейшие рассуждения повторяют доказательство теоремы 9.2. Получаем противоречие с тем фактом, что $\left(u_{0}, u_{1}\right) \in M^{2}$.

Теперь докажем, что при $q \geqslant 2$ в некотором классе начальных функций единственное слабое решение задачи Коши (7.3), (7.4) в смысле определения 7.3 разрушается за конечное время.

Определение 10.2. Будем говорить, что начальные функции $u_{0}, u_{1}$ принадлежат классу $N^{2}$ (обозначение $\left.\left(u_{0}, u_{1}\right) \in N^{2}\right)$, если найдется такая пробная функция $\phi(x, t) \in \mathscr{D}\left(\mathbb{R}^{3} \otimes(-\infty,+\infty)\right.$ ), что $\phi_{t}(x, t) \leqslant 0$ для всех $(x, t) \in \mathbb{R}^{3} \otimes[0,+\infty)$, конечна нелинейная емкость

$$
E_{2}(\phi):=\int_{0}^{+\infty} \int_{\mathbb{R}^{3}} \frac{\left|\nabla \frac{\partial^{2} \phi(x, t)}{\partial t^{2}}\right|^{q^{\prime}}}{\left(-\frac{\partial \phi(x, t)}{\partial t}\right)^{q^{\prime} / q}} d x d t+\sum_{j=1}^{3} \omega_{j}^{2} \int_{0}^{+\infty} \int_{\mathbb{R}^{3}} \frac{\left|\phi_{x_{j}}(x, t)\right|^{q^{\prime}}}{\left(-\frac{\partial \phi(x, t)}{\partial t}\right)^{q^{\prime} / q}} d x d t<+\infty
$$

и при этом выполнено неравенство

$$
\begin{aligned}
\frac{1}{q^{\prime}}\left(\frac{2}{q}\right)^{q^{\prime} / q} E_{2}(\phi)-\int_{\mathbb{R}^{3}}\left[\left(\nabla \frac{\partial \phi}{\partial t}(x, 0), \nabla\right.\right. & \left.u_{0}(x)\right)- \\
& \left.-\left(\nabla \phi(x, 0), \nabla u_{1}(x)\right)-\phi(x, 0)\left|\nabla u_{0}(x)\right|^{q}\right] d x<0 .
\end{aligned}
$$

Замечание 10.2. Такая пробная функция $\phi(x, t)$, что конечна емкость (10.43) существует; это доказано в [14]. Фиксируем эту пробную функцию. Если вместо $u_{1}(x)$ рассмотреть функцию $r u_{1}(x)$, то за счет большой величины $|r|>0$ при некоторой фиксированной функции $u_{0}(x)$ можно добиться выполнения неравенства (10.44).

Теорема 10.3. Пусть $q \geqslant 2 u\left(u_{0}, u_{1}\right) \in N^{2}$. Тогда существует единственное локальное во времени слабое решение задачи Коши (7.3), (7.4) в смысле определения 7.3 в классе $\mathbb{C}^{(1)}\left([0, T] ; \mathbb{C}_{b}^{1,1 / 2}\left(\left(1+|x|^{2}\right) ; \mathbb{R}^{3}\right)\right)$ для всех $T \in\left(0, T_{0}\right)$ класса гладкости $\mathbb{C}^{(2)}\left([0, T] ; \mathbb{C}_{b}^{1,1 / 2}((1+\right.$ $\left.\left.|x|^{2}\right) ; \mathbb{R}^{3}\right)$ ), причем $0<T_{0}<+\infty$ и слабое решение разрушается в момент времени $T_{0}$ в следующем смысле:

$$
\lim _{T \uparrow T_{0}}\left\|\left(1+|x|^{2}\right)^{1 / 2} u(x, t)\right\|_{1, T}=+\infty .
$$


Доказательство повторяет доказательство теоремы 9.3 и основано на применении к слагаемым из равенства (7.10) из определения 7.4 глобального во времени слабого решения задачи Коши (7.3), (7.4).

11. ЗАДАЧА КОШИ (7.5), (7.6): СУЩЕСТВОВАНИЕ ЛОКАЛЬНЫХ И ОТСУТСТВИЕ ЛОКАЛЬНЫХ И ГЛОБАЛЬНЫХ ВО ВРЕМЕНИ СЛАБЫХ РЕШЕНИЙ

Рассмотрим вспомогательное интегральное уравнение

$$
\begin{aligned}
u(x, t)=-\int_{0} \int_{\mathbb{R}^{3}} \mathscr{E}(x-y, t-\tau) \frac{\partial^{2}|\nabla u(y, \tau)|^{q}}{\partial \tau^{2}} d y d \tau+ & \\
& +\int_{\mathbb{R}^{3}} \frac{\partial \mathscr{E}(x-y, t)}{\partial t} \Delta u_{0}(y) d y+\int_{\mathbb{R}^{3}} \mathscr{E}(x-y, t) \Delta u_{1}(y) d y .
\end{aligned}
$$

Перейдя в уравнении (11.1) к новой функции (9.2), получим интегральное уравнение

$$
\begin{aligned}
v(x, t)=\int_{0} \int_{\mathbb{R}^{3}} G_{q}(x, y, t-\tau) \rho(y, \tau) d y & d \tau+\int_{\mathbb{R}^{3}} \frac{\partial G_{\alpha}(x, y, t)}{\partial t} \mu_{\alpha}(y) d y+ \\
& +\int_{\mathbb{R}^{3}} G_{\beta}(x, y, t) \sigma_{\beta}(y) d y:=U(x, t)+W(x, t)+V(x, t),
\end{aligned}
$$

где

$$
\begin{gathered}
G_{\gamma}(x, y, t):=\frac{\left(1+|x|^{2}\right)^{1 / 2}}{\left(1+|y|^{2}\right)^{\gamma}} \mathscr{E}(x-y, t), \\
\rho(x, t):=-\frac{\partial^{2}}{\partial t^{2}}\left|\left(1+|x|^{2}\right)^{1 / 2} \nabla v(x, t)-\frac{x}{\left(1+|x|^{2}\right)^{1 / 2}} v(x, t)\right|^{q}, \\
\mu_{\alpha}(x):=\left(1+|x|^{2}\right)^{\alpha} \Delta u_{0}(x), \quad \sigma_{\beta}(x):=\left(1+|x|^{2}\right)^{\beta} \Delta u_{1}(x) .
\end{gathered}
$$

Лемма 11.1. Пусть $q=2 u v(x, t) \in \mathbb{C}^{(2)}\left([0, T] ; \mathbb{C}_{b}^{1,0}\left(\left(1+\mid x^{2}\right)^{1 / 2} ; \mathbb{R}^{3}\right)\right)$. Тогда функиия $\rho(x, t)$ принадлежст классу $\mathbb{C}\left([0, T] ; \mathbb{C}_{b}\left(\mathbb{R}^{3}\right)\right)$, причем для любъх $v_{k}(x, t) \in \mathbb{C}^{(2)}\left([0, T] ; \mathbb{C}_{b}^{1,0}((1+\right.$ $\left.\left.\left(x^{2}\right)^{1 / 2} ; \mathbb{R}^{3}\right)\right)$ при $k=1,2$ справедливо следующее неравенство:

$$
\begin{gathered}
\sup _{t \in[0, T]}\left|\rho_{1}(x, t)-\rho_{2}(x, t)\right|_{0} \leqslant 6 \max \left\{\left\|v_{1}\right\|_{2, T},\left\|v_{2}\right\|_{2, T}\right\}\left\|v_{1}-v_{2}\right\|_{2, T}, \\
\rho_{k}(x, t):=-\frac{\partial^{2}}{\partial t^{2}}\left|\left(1+|x|^{2}\right)^{1 / 2} \nabla v_{k}(x, t)-\frac{x}{\left(1+|x|^{2}\right)^{1 / 2}} v_{k}(x, t)\right|^{2}, \quad k=1,2,
\end{gathered}
$$

где

$$
\|v\|_{2, T}:=\|v\|_{0, T}+\left\|\frac{\partial v}{\partial t}\right\|_{0, T}+\left\|\frac{\partial^{2} v}{\partial t^{2}}\right\|_{0, T}, \quad\|w\|_{0, T}:=\sup _{t \in[0, T]}|w(x, t)|_{1} .
$$

Доказательство. Справедливы следующие соотношения:

$$
\begin{gathered}
\frac{\partial^{2}}{\partial t^{2}}|z(x, t)|^{2}=2\left|z_{t}(x, t)\right|^{2}+2\left(z_{t t}(x, t), z(x, t)\right), \\
\left.|| z_{1 t}\right|^{2}-\left|z_{2 t}\right|^{2}\left|\leqslant 2 \max \left\{\left|z_{1 t}\right|,\left|z_{2 t}\right|\right\}\right| z_{1 t}-z_{2 t} \mid, \\
\left|\left(z_{1 t t}, z_{1}\right)-\left(z_{2 t t}, z_{2}\right)\right| \leqslant\left|z_{1 t t}\right|\left|z_{1}-z_{2}\right|+\left|z_{2}\right|\left|z_{1 t t}-z_{2 t t}\right| .
\end{gathered}
$$

Из (11.9) с учетом (11.10) и (11.11) вытекает следующая оценка: 


$$
\begin{aligned}
& \left.\left|\frac{\partial^{2}}{\partial t^{2}}\right| z_{1}\right|^{2}-\frac{\partial^{2}}{\partial t^{2}}\left|z_{2}\right|^{2} \mid \leqslant \\
& \quad \leqslant 6 \max \left\{\left|z_{1}\right|+\left|z_{1 t}\right|+\left|z_{1 t t}\right|,\left|z_{2}\right|+\left|z_{2 t}\right|+\left|z_{2 t t}\right|\right\}\left[\left|z_{1}-z_{2}\right|+\left|z_{1 t}-z_{2 t}\right|+\left|z_{1 t t}-z_{2 t t}\right|\right] .
\end{aligned}
$$

Из этого неравенства вытекают все утверждения леммы.

Относительно разрешимости интегрального уравнения (11.2) справедливо следующее утверждение.

Лемма 11.2. Пусть $q=2$. Тогда для любого $T>0$ найдутся такие малые $R_{1}>0, R_{2}>0$ u $R>0$, что для любых функиий $u_{0}(x) \in \mathbb{C}_{b}^{(2)}\left(\left(1+|x|^{2}\right)^{\alpha} ; \mathbb{R}^{3}\right), u_{1}(x) \in \mathbb{C}_{b}^{(2)}\left(\left(1+|x|^{2}\right)^{\beta} ; \mathbb{R}^{3}\right)$ nри условиях $\alpha>3 / 2, \beta>3 / 2$, для которых справедливы неравенства

$$
\left|u_{0}\right|_{2} \leqslant R_{1}, \quad\left|u_{1}\right|_{2} \leqslant R_{2}
$$

существует единственное решение $v(x, t)$ интегрального уравнения (11.2) в шаре

$$
D_{R, T}:=\left\{v(x, t) \in \mathbb{C}^{(2)}\left([0, T] ; \mathbb{C}_{b}^{1,0}\left(\left(1+|x|^{2}\right)^{1 / 2} ; \mathbb{R}^{3}\right)\right):\|v(x, t)\|_{2, T} \leqslant R\right\} .
$$

Доказательство. Прежде всего заметим, что в отличие от уравнений (7.1) и (7.3), в уравнении (7.5) порядок производной по времени от нелинейности совпадает с порядком производной от функции $u(x, t)$ линейной части уравнения (7.5). Поэтому для соответствующих интегральных уравнений (9.4) и (10.2) удалось доказать леммы 9.2 и 10.2 о существовании непродолжаемых решений в соответствующих банаховых пространствах. Однако для интегрального уравнения (11.2) получить результат о существовании непродолжаемого решений нам не удалось. Связано с этим, что в классе $v(x, t) \in \mathbb{C}^{(2)}\left([0, T] ; \mathbb{C}_{b}^{1,0}\left(\left(1+|x|^{2}\right)^{1 / 2} ; \mathbb{R}^{3}\right)\right)$ нам необходимо оценить вторую производную по времени от потенциала $U(x, t)$, которая имеет следующий явный вид:

$$
\frac{\partial^{2} U(x, t)}{\partial t^{2}}=-\int_{\mathbb{R}^{3}} \frac{\left(1+|x|^{2}\right)^{1 / 2}}{\left(1+|y|^{2}\right)^{q}} \frac{1}{4 \pi|x-y|} \rho(y, t) d y+\int_{0}^{t} \int_{\mathbb{R}^{3}} \frac{\partial^{2} G_{q}(x, y, t-\tau)}{\partial t^{2}} \rho(y, \tau) d y d \tau,
$$

где плотность $\rho(x, t)$ определена равенством (11.4). Наличие «стационарного» потенциала

$$
V_{0}(x, t)=-\int_{\mathbb{R}^{3}} \frac{\left(1+|x|^{2}\right)^{1 / 2}}{\left(1+|y|^{2}\right)^{q}} \frac{1}{4 \pi|x-y|} \rho(y, t) d y
$$

не дает возможности воспользоваться стандартным алгоритмом продолжения решения во времени. С другой стороны, несложно воспользоваться леммой 11.1 и теоремами 8.1-8.3 и доказать утверждение настоящей леммы при помощи метода сжимающих отображений.

Из леммы 11.2 вытекает следующее утверждение относительно разрешимости интегрального уравнения (11.1).

Лемма 11.3. Пусть $q=2$. Тогда для любого $T>0$ найдутся такие малье $R_{1}>0, R_{2}>0$ u $R>0$, что для любых функций $u_{0}(x) \in \mathbb{C}_{b}^{(2)}\left(\left(1+|x|^{2}\right)^{\alpha} ; \mathbb{R}^{3}\right), u_{1}(x) \in \mathbb{C}_{b}^{(2)}\left(\left(1+|x|^{2}\right)^{\beta} ; \mathbb{R}^{3}\right)$ nри условиях $\alpha>3 / 2, \beta>3 / 2$, для которых справедливы неравенства

$$
\left|u_{0}\right|_{2} \leqslant R_{1}, \quad\left|u_{1}\right|_{2} \leqslant R_{2},
$$

существует единственное решение $и(x, t)$ интегрального уравнения (11.1) в шаре

$$
B_{R, T}:=\left\{u(x, t) \in \mathbb{C}^{(2)}\left([0, T] ; \mathbb{C}_{b}^{1,1 / 2}\left(\left(1+|x|^{2}\right) ; \mathbb{R}^{3}\right)\right):\left\|\left(1+|x|^{2}\right)^{1 / 2} u(x, t)\right\|_{2, T} \leqslant R\right\} .
$$

Из леммы 11.3 и равенства (9.3) вытекает, что

$$
\begin{gathered}
|\nabla u(x, t)|^{2} \in \mathbb{C}^{(2)}\left([0, T] ; \mathbb{C}_{b}\left(\left(1+|x|^{2}\right)^{2} ; \mathbb{R}^{3}\right)\right) \subset \mathbb{C}_{x, t}^{(0,2)}\left(\mathbb{R}^{3} \otimes[0, T]\right), \\
\rho_{1}(x, t)=-\frac{\partial^{2}}{\partial t^{2}}|\nabla u(x, t)|^{2} \in \mathbb{C}\left([0, T] ; \mathbb{C}_{b}\left(\left(1+|x|^{2}\right)^{2} ; \mathbb{R}^{3}\right)\right) .
\end{gathered}
$$

Поэтому из леммы 11.3 , свойств (11.19) и (11.20) и теорем 7.3 и 8.5 вытекает следующее утверждение. 
Теорема 11.1. Пусть $q=2$. Тогда для любого $T>0$ найдутся такие малье $R_{1}>0, R_{2}>0$, $R>0$, что для любых функиий $u_{0}(x) \in \mathbb{C}_{b}^{(2)}\left(\left(1+|x|^{2}\right)^{\alpha} ; \mathbb{R}^{3}\right), u_{1}(x) \in \mathbb{C}_{b}^{(2)}\left(\left(1+|x|^{2}\right)^{\beta} ; \mathbb{R}^{3}\right), \alpha>3 / 2$, $\beta>3 / 2$, для которых выполнены неравенства

$$
\left|u_{0}\right|_{2} \leqslant R_{1}, \quad\left|u_{1}\right|_{2} \leqslant R_{2},
$$

существует единственное слабое локальное во времени решение $u(x, t)$ задачи Коши (7.5), (7.6) в смысле определения 7.5 в классе $\mathbb{C}^{(2)}\left([0, T] ; \mathbb{C}_{b}^{1,1 / 2}\left(\left(1+|x|^{2}\right) ; \mathbb{R}^{3}\right)\right) \cap B_{R, T}$, где

$$
B_{R, T}:=\left\{u(x, t) \in \mathbb{C}^{(2)}\left([0, T] ; \mathbb{C}_{b}^{1,1 / 2}\left(\left(1+|x|^{2}\right) ; \mathbb{R}^{3}\right)\right):\left\|\left(1+|x|^{2}\right)^{1 / 2} u(x, t)\right\|_{2, T} \leqslant R\right\},
$$

причем и $(x, t)$ удовлетворяет начальным условиям

$$
u(x, 0)=u_{0}(x), \quad \frac{\partial u}{\partial t}(x, 0)=u_{1}(x) \quad \text { для всех } x \in \mathbb{R}^{3} .
$$

Лемма 11.4. Пусть и $(x, t)$ - слабое локальное во времени решение задачи Коши (7.5), (7.6) в смысле определения 7.5. Тогда равенство (7.18) справедливо для любой функиии $\phi(x, t)=$ $\phi_{1}(x) \phi_{2}(t)$, где $\phi_{1}(x) \in \mathscr{D}\left(\mathbb{R}^{3}\right) u \phi_{2}(t) \in \mathbb{C}_{0}^{(2)}[0, T]$.

Доказательство в точности повторяет доказательство леммы 9.4.

Лемма 11.5. Если $1<q \leqslant 3 / 2$ и и(x,t) - локальное во времени слабое решение задачи Коши (7.5), (7.6) в смысле определения 7.5, с начальными функциями $u_{0}(x), u_{1}(x) \in W_{q}^{1}\left(\mathbb{R}^{3}\right)$. Тогда справедлива следующая априорная ощенка:

$$
\begin{aligned}
& \int_{\mathbb{R}^{3}}\left[\frac{\lambda}{T}\left|\nabla u_{0}(x)\right|^{q}+q\left(\nabla u_{1}(x),\left|\nabla u_{0}(x)\right|^{q-2} \nabla u_{0}(x)\right)\right] d x \geqslant \\
& \geqslant \frac{\lambda(\lambda-1)}{T^{2}} \int_{0}^{T} \int_{\mathbb{R}^{3}}\left(1-\frac{t}{T}\right)^{\lambda-2}|\nabla u(x, t)|^{q} d x d t, \quad \lambda>2 .
\end{aligned}
$$

Доказательство. С учетом леммы 11.4 возьмем в равенстве (7.18) пробную функцию вида (9.36)(9.38). Далее, используя неравенства Гельдера, точно так же, как при доказательстве теоремы 9.2 приходим после предельного перехода при $R \rightarrow+\infty$ к неравенству (11.24).

Теорема 11.2. Если $1<q \leqslant 3 / 2 u u_{0}(x), u_{1}(x) \in W_{q}^{1}\left(\mathbb{R}^{3}\right)$, то при условии

$$
\int_{\mathbb{R}^{3}}\left(\nabla u_{1}(x),\left|\nabla u_{0}(x)\right|^{q-2} \nabla u_{0}(x)\right) d x<0
$$

глобальное во времени слабое решение и(x,t) задачи Коши (7.5), (7.6) в смысле определения 7.6 отсутствует. Более того, для времени существования локального во времени слабого решения задачи Коши (7.5), (7.6) в смысле определения 7.5 справедлива следующая оценка:

$$
T \leqslant-\frac{2}{q} \frac{I_{1}}{I_{2}}, \quad I_{1}:=\int_{\mathbb{R}^{3}}\left|\nabla u_{0}(x)\right|^{q} d x, \quad I_{2}:=\int_{\mathbb{R}^{3}}\left(\nabla u_{1}(x),\left|\nabla u_{0}(x)\right|^{q-2} \nabla u_{0}(x)\right) d x .
$$

Если же начальные функиии $\left(u_{0}, u_{1}\right) \in M^{2}$, то локальные во времени слабые решения задачи Коши (7.5), (7.6) в смысле определения 7.5 отсутствуют для любого $T>0$.

Доказательство основано на несложном анализе априорной оценки (11.24). При этом вторая часть утверждения теоремы доказывается точно так же, как соответствующая часть теоремы 9.2. 


\section{2. ПРИЛОЖЕНИЕ}

Изучим вопрос о плотности вложения $\mathscr{D}(-\infty, T)$ в банахово пространство $\mathbb{C}_{0}^{(2)}[0, T]$.

Пусть $\phi(t) \in \mathbb{C}_{0}^{(2)}[0, T]:=\left\{\phi(t) \in \mathbb{C}^{(2)}[0, T]: \phi(T)=\phi^{\prime}(T)=\phi^{\prime \prime}(T)=0\right\} ;$ это пространство, очевидно, является банаховым пространством относительно стандартной нормы

$$
\|\phi\|:=\sup _{t \in[0, T]}|\phi(t)|+\sup _{t \in[0, T]}\left|\phi^{\prime}(t)\right|+\sup _{t \in[0, T]}\left|\phi^{\prime \prime}(t)\right| .
$$

Теорема 12.1. Имеет место плотное вложение $\mathscr{D}(-\infty, T) \stackrel{d s}{\subset} \mathbb{C}_{0}^{(2)}[0, T]$.

Доказательство. Продолжим произвольно фиксированную функцию $\phi(t) \in \mathbb{C}_{0}^{(2)}[0, T]$ на всю прямую $\mathbb{R}^{1}$ следующим образом:

$$
\bar{\phi}(t)= \begin{cases}\phi(t), & t \in[0, T] \\ 0, & t \geqslant T, t \leqslant-T_{1}, T_{1}>0,\end{cases}
$$

и при этом $\bar{\phi}(t) \in \mathbb{C}_{0}^{(2)}\left(\mathbb{R}^{1}\right)$. Такое продолжение существует в силу определения пространства $\mathbb{C}_{0}^{(2)}[0, T]$. Пусть $\varepsilon \in(0, T / 2)$. Рассмотрим функцию

$$
\bar{\phi}_{\varepsilon}(t):=\int_{-\infty}^{T-2 \varepsilon} \omega_{\varepsilon}(|t-s|) \bar{\phi}(s) d s
$$

где

$$
\omega_{\varepsilon}(|t|):=\frac{a}{\varepsilon} \begin{cases}\exp \left(-\frac{\varepsilon^{2}}{\varepsilon^{2}-t^{2}}\right), & |t| \leqslant \varepsilon, \\ 0, & |t|>\varepsilon,\end{cases}
$$

а постоянная $a>0$ определяется из условия нормировки

$$
\int_{\mathbb{R}^{1}} \omega_{\varepsilon}(|t|) d t=1
$$

Заметим, что $\bar{\phi}_{\varepsilon}(t) \in \mathscr{D}(-\infty, T)$. Справедлива следующая цепочка равенств:

$$
\begin{aligned}
\bar{\phi}(t)-\bar{\phi}_{\varepsilon}(t)= & \bar{\phi}(t)-\int_{-\infty}^{T+2 \varepsilon} \omega_{\varepsilon}(|t-s|) \bar{\phi}(s) d s+\int_{T-2 \varepsilon}^{T+2 \varepsilon} \omega_{\varepsilon}(|t-s|) \bar{\phi}(s) d s= \\
& =\bar{\phi}(t)-\int_{-\infty}^{+\infty} \omega_{\varepsilon}(|t-s|) \bar{\phi}(s) d s+\int_{T-2 \varepsilon}^{T} \omega_{\varepsilon}(|t-s|) \phi(s) d s= \\
& =\int_{-\infty}^{+\infty} \omega_{\varepsilon}(|t-s|)[\bar{\phi}(t)-\bar{\phi}(s)] d s+\int_{T-2 \varepsilon}^{T} \omega_{\varepsilon}(|t-s|) \phi(s) d s:=I_{1}(\varepsilon, t)+I_{2}(\varepsilon, t) .
\end{aligned}
$$

Справедливы следующие оценки:

$$
\begin{aligned}
& \sup _{t \in[0, T]}\left|I_{1}(\varepsilon, t)\right| \leqslant \sup _{t \in[0, T]|t-s| \leqslant \varepsilon} \sup _{t \in[}|\bar{\phi}(t)-\overline{\phi(s)}|, \\
& \sup _{t \in[0, T]}\left|I_{2}(\varepsilon, t)\right| \leqslant \sup _{s \in[T-2 \varepsilon, T]}|\phi(s)-\phi(T)| .
\end{aligned}
$$

Имеем $\bar{\phi}(t) \in \mathbb{C}_{0}^{(2)}\left(\mathbb{R}^{1}\right) \subset \mathbb{C}\left(\mathbb{R}^{1}\right), \phi(t) \in \mathbb{C}_{0}^{(2)}[0, T]$. Поэтому из (12.6)-(12.8) вытекает, что для любого $\delta>0$ найдется такое $\varepsilon=\varepsilon(\delta)>0$, что

$$
\sup _{t \in[0, T]}\left|\phi(t)-\bar{\phi}_{\varepsilon}(t)\right|=\sup _{t \in[0, T]}\left|\bar{\phi}(t)-\bar{\phi}_{\varepsilon}(t)\right|<\delta .
$$


Теперь заметим, что справедлива следующая цепочка соотношений:

$$
\begin{aligned}
\bar{\phi}^{\prime}(t)-\bar{\phi}_{\varepsilon}^{\prime}(t)=\int_{-\infty}^{+\infty} \omega_{\varepsilon}(|t-s|)\left[\bar{\phi}^{\prime}(t)-\bar{\phi}^{\prime}(s)\right] d s+\int_{T-2 \varepsilon}^{T+2 \varepsilon} \frac{d \omega_{\varepsilon}(|t-s|)}{d t} \bar{\phi}(s) d s= \\
=\int_{-\infty}^{+\infty} \omega_{\varepsilon}(|t-s|)\left[\bar{\phi}^{\prime}(t)-\bar{\phi}^{\prime}(s)\right] d s+\int_{T-2 \varepsilon}^{T} \omega_{\varepsilon}(|t-s|) \phi^{\prime}(s) d s+ \\
\quad+\omega_{\varepsilon}(|t+2 \varepsilon-T|) \phi(T-2 \varepsilon):=I_{3}(\varepsilon, t)+I_{4}(\varepsilon, t)+I_{5}(\varepsilon, t) .
\end{aligned}
$$

Справедливы следующие оценки:

$$
\begin{gathered}
\sup _{t \in[0, T]}\left|I_{3}(\varepsilon, t)\right| \leqslant \sup _{t \in[0, T]|t-s| \leqslant \varepsilon} \sup _{\mid}\left|\bar{\phi}^{\prime}(t)-\bar{\phi}^{\prime}(s)\right|, \\
\sup _{t \in[0, T]}\left|I_{4}(\varepsilon, t)\right| \leqslant \sup _{t \in[T-2 \varepsilon, T]}\left|\phi^{\prime}(s)-\phi^{\prime}(T)\right|, \\
\sup _{t \in[0, T]}\left|I_{5}(\varepsilon, t)\right| \leqslant \frac{a}{\varepsilon} \sup _{t \in[0, T]} \omega\left(\frac{|t+2 \varepsilon-T|}{\varepsilon}\right) 2 \varepsilon^{2} \sup _{\eta \in[T-2 \varepsilon, T]}\left|\phi^{\prime \prime}(\eta)\right| ;
\end{gathered}
$$

в (12.13) мы воспользовались разложением

$$
\phi(T-2 \varepsilon)=\phi(T)-2 \varepsilon \phi^{\prime}(T)+2 \varepsilon^{2} \phi^{\prime \prime}(\eta), \quad \eta \in[T-2 \varepsilon, T],
$$

причем $\phi(T)=\phi^{\prime}(T)=\phi^{\prime \prime}(T)=0$. Таким образом, для любого $\delta>0$ найдется такое $\varepsilon=\varepsilon(\delta)>0$, что из (12.10)-(12.14) вытекает оценка

$$
\sup _{t \in[0, T]}\left|\phi^{\prime}(t)-\bar{\phi}_{\varepsilon}^{\prime}(t)\right|=\sup _{t \in[0, T]}\left|\bar{\phi}^{\prime}(t)-\bar{\phi}_{\varepsilon}^{\prime}(t)\right|<\delta .
$$

Справедлива следующая цепочка соотношений:

$$
\begin{aligned}
& \bar{\phi}^{\prime \prime}(t)-\bar{\phi}_{\varepsilon}^{\prime \prime}(t)=\int_{-\infty}^{+\infty} \omega_{\varepsilon}(|t-s|)\left[\bar{\phi}^{\prime \prime}(t)-\bar{\phi}^{\prime \prime}(s)\right] d s+\int_{T-2 \varepsilon}^{T+2 \varepsilon} \frac{d^{2} \omega_{\varepsilon}(|t-s|)}{d t^{2}} \bar{\phi}(s) d s, \\
& \int_{T-2 \varepsilon}^{T+2 \varepsilon} \frac{d^{2} \omega_{\varepsilon}(|t-s|)}{d t^{2}} \bar{\phi}(s) d s=\int_{T-2 \varepsilon}^{T} \frac{d^{2} \omega_{\varepsilon}(|t-s|)}{d s^{2}} \phi(s) d s= \\
& =-\left.\frac{d \omega_{\varepsilon}(|t-s|)}{d s}\right|_{s=T-2 \varepsilon} \phi(T-2 \varepsilon)+\omega_{\varepsilon}(|t-T+2 \varepsilon|) \phi^{\prime}(T-2 \varepsilon)+\int_{T-2 \varepsilon}^{T} \omega_{\varepsilon}(|t-2|) \phi^{\prime \prime}(s) d s .
\end{aligned}
$$

Из равенств (12.16) и (12.17) вытекает следующее равенство:

$$
\bar{\phi}^{\prime \prime}(t)-\bar{\phi}_{\varepsilon}^{\prime \prime}(t)=I_{6}(\varepsilon, t)+I_{7}(\varepsilon, t)+I_{8}(\varepsilon, t)+I_{9}(\varepsilon, t),
$$

где

$$
\begin{gathered}
I_{6}(\varepsilon, t):=\int_{-\infty}^{+\infty} \omega_{\varepsilon}(|t-s|)\left[\bar{\phi}^{\prime \prime}(t)-\bar{\phi}^{\prime \prime}(s)\right] d s, \\
I_{7}(\varepsilon, t):=-\left.\frac{d \omega_{\varepsilon}(|t-s|)}{d s}\right|_{s=T-2 \varepsilon} \phi(T-2 \varepsilon), \\
I_{8}(\varepsilon, t):=\omega_{\varepsilon}(|t-T+2 \varepsilon|) \phi^{\prime}(T-2 \varepsilon), \\
I_{9}(\varepsilon, t):=\int_{T-2 \varepsilon}^{T} \omega_{\varepsilon}(|t-2|) \phi^{\prime \prime}(s) d s .
\end{gathered}
$$


Справедливы следующие оценки:

$$
\begin{gathered}
\sup _{t \in[0, T]}\left|I_{6}(\varepsilon, t)\right| \leqslant \sup _{t \in[0, T]} \sup _{|t-s| \leqslant \varepsilon}\left|\bar{\phi}^{\prime \prime}(t)-\bar{\phi}^{\prime \prime}(s)\right|, \\
\sup _{t \in[0, T]}\left|I_{7}(\varepsilon, t)\right| \leqslant \frac{A}{\varepsilon^{2}} 2 \varepsilon^{2} \sup _{\eta \in[T-2 \varepsilon, T]}\left|\phi^{\prime \prime}(\eta)\right|, \\
\sup _{t \in[0, T]}\left|I_{8}(\varepsilon, t)\right| \leqslant \frac{A}{\varepsilon} 2 \varepsilon \sup _{\eta \in[T-2 \varepsilon, T]}\left|\phi^{\prime \prime}(\eta)\right|, \\
\sup _{t \in[0, T]}\left|I_{9}(\varepsilon, t)\right| \leqslant \sup _{\eta \in[T-2 \varepsilon, T]}\left|\phi^{\prime \prime}(\eta)\right|,
\end{gathered}
$$

где мы воспользовались разложением (12.14). Осталось заметить, что $\phi(t) \in \mathbb{C}_{0}^{(2)}[0, T]$ и поэтому

$$
\sup _{\eta \in[T-2 \varepsilon, T]}\left|\phi^{\prime \prime}(\eta)\right| \rightarrow+0 \quad \text { при } \varepsilon \rightarrow+0 .
$$

Таким образом, из соотношений (12.18)-(12.27) вытекает, что для любого $\delta>0$ найдется такое $\varepsilon=\varepsilon(\delta)>0$, что справедливо следующее соотношение:

$$
\sup _{t \in[0, T]}\left|\phi^{\prime \prime}(t)-\bar{\phi}_{\varepsilon}^{\prime \prime}(t)\right|=\sup _{t \in[0, T]}\left|\bar{\phi}^{\prime \prime}(t)-\bar{\phi}_{\varepsilon}^{\prime \prime}(t)\right|<\delta .
$$

Из неравенств $(12.9),(12.15)$ и (12.28) вытекает, что для любого $\delta>0$ найдется такое $\varepsilon=\varepsilon(\delta)>0$, что справедливо неравенство

Таким образом, теорема доказана.

$$
\left\|\phi(t)-\bar{\phi}_{\varepsilon}(t)\right\|<3 \delta
$$

\section{СПИСОК ЛИТЕРАТУРЫ}

1. Владимиров B. С. Уравнения математической физики. - М.: Наука, 1988.

2. Габов C. A. Новые задачи математической теории волн. - М.: Физматлит, 1998.

3. Габов C. A., Свешников A. Г. Линейные задачи теории нестационарных внутренних волн. - М.: Наука, 1990.

4. Галахов Е. И., Салиева О. А. Об отсутствии неотрицательных монотонных решений для некоторых коэрцитивных неравенств в полупространстве// Совр. мат. Фундам. напр. - 2017. — 63, № 4. - С. 573585.

5. Двайт Г. Б. Таблицы интегралов и другие математические формулы. - М.: Наука, 1964.

6. Загребина C. A. Начально-конечная задача для уравнений соболевского типа с сильно $(L, p)-$ радиальным оператором// Мат. заметки ЯГУ. - 2012. - 19, № 2. - С. 39-48.

7. Капитонов Б. В. Теория потенциала для уравнения малых колебаний вращающейся жидкости// Мат. сб. - 1979. - 109 (151), № 4 (8). - С. 607-628.

8. Корпусов М. О. Критические показатели мгновенного разрушения или локальной разрешимости нелинейных уравнений соболевского типа// Изв. РАН. Сер. мат. - 2015. - 79, № 5. - С. $103-162$.

9. Корпусов M. О. О разрушении решений нелинейных уравнений типа уравнения ХохловаЗаболотской// Теор. мат. физ. - 2018. - 194, № 3. - С. 403-417.

10. Корпусов М. О., Панин А. А., Шишков А. Е. О критическом показателе «мгновенное разрушение» versus «локальная разрешимость» в задаче Коши для модельного уравнения соболевского типа// Изв. РАН. Сер. мат. - 2021. - 85, № 1. - С. 118-153.

11. Корпусов М. О., Плетнер Ю. Д., Свешников А. Г. О нестационарных волнах в средах с анизотропной дисперсией // Ж. вычисл. мат. мат. физ. - 1999. - 39, № 6. - С. 1006-1022.

12. Панин A. А. О локальной разрешимости и разрушении решения абстрактного нелинейного интегрального уравнения Вольтерра// Мат. заметки. - 2015. - 97, № 6. - С. 884-903.

13. Плетнер Ю. Д. Фундаментальные решения операторов типа Соболева и некоторые начально-краевые задачи // Ж. вычисл. мат. мат. физ. - 1992. - 32, № 12. - С. 1885-1899.

14. Похожаев С. И., Митидиери Э. Априорные оценки и отсутствие решений нелинейных уравнений и неравенств в частных производных// Тр. Мат. ин-та им. В. А. Стеклова. - 2001. - 234. - С. 3-383.

15. Свиридюк Г. А. К общей теории полугрупп операторов// Усп. мат. наук. - 1994. - 49, № 4. - С. $47-74$. 
16. Galakhov E. I. Some nonexistence results for quasilinear elliptic problems// J. Math. Anal. Appl. — 2000. - 252, № 1. - P. 256-277.

17. Korpusov M. O., Ovchinnikov A. V., Panin A. A. Instantaneous blow-up versus local solvability of solutions to the Cauchy problem for the equation of a semiconductor in a magnetic field// Math. Methods Appl. Sci. - 2018. - 41, № 17. - P. 8070-8099.

18. Zamyshlyaeva A. A., Sviridyuk G. A. Nonclassical equations of mathematical physics. Linear Sobolev-type equations of higher order// Вестн. ЮУрГУ. Сер. мат. мех. физ. - 2016. - 8, № 4. - С. 5-16.

Корпусов Максим Олегович

Московский государственный университет им. М. В. Ломоносова

E-mail: korpusov@gmail.com

Шляпугин Григорий Игоревич

Московский государственный университет им. М. В. Ломоносова

E-mail: g.shlypugin@yandex.ru 